\title{
The Role of Ghrelin in Reward-Based Feeding
}

\author{
by \\ Véronique St-Onge
}

A thesis submitted to the Faculty of Graduate and Postdoctoral
Affairs in partial fulfillment of the requirements for the degree of

Doctor in Philosophy

in

Neuroscience

Carleton University

Ottawa, Ontario

(C) 2014, Véronique St-Onge 


\section{Abstract}

The incidence of obesity has nearly doubled in the last 30 years, and this rise in incidence is directly linked to increased consumption of energy-dense foods. In addition, the majority of people attempting to lose weight report relapse to high fat foods while dieting. Recent studies suggest that the orexigenic hormone ghrelin can bind to dopaminergic cells in the ventral tegmental area (VTA), a key structure in the brain's reward system, and increase the incentive value of such foods. Specifically, studies have shown that ghrelin increases the motivation to work for food rewards, enhances the preference for contexts associated with food rewards, and augments the locomotor and physiological responses associated with food rewards. The experiments described in the present thesis aimed to establish the role of ghrelin in 2 additional reward-based feeding behaviours thought to contribute to the current obesity epidemic. The first behaviour is the consumption of palatable desserts after meals that adequately satisfy an individual's energetic demands. The second behaviour is the tendency to relapse to food seeking after a period of abstinence. In order to accomplish this, we compared ghrelin receptor knock out $(\mathrm{KO})$ rats to rats possessing an identical genetic background, with an intact ghrelin receptor gene (wildtype rats - WT) in tasks designed to model these behaviours. In addition, we examined outbred rats receiving chronic infusions of either ghrelin or a ghrelin receptor antagonist in the VTA. Overall, the data presented in this thesis confirmed the role of ghrelin signalling in dessert consumption; KO rats ate less cookie dough when sated than WT rats. KO and WT rats performed similarly in operant conditioning and conditioned place preference models of relapse, also known as the operant and CPP reinstatement tests. In contrast, pharmacological action at the ghrelin 
receptor in outbred rats could enhance and attenuate the expression of reinstatement of food seeking that is induced by food reward cues following administration of ghrelin and a ghrelin receptor antagonist, respectively. It is therefore likely that ghrelin is involved in the tendency to relapse to unhealthy eating habits in dieting individuals, and that this effect is due, in part, to its action in the reward system, particularly within the VTA. 


\section{Acknowledgements}

First, I would like to thank my supervisor, Dr. Alfonso Abizaid, whose incredible mentorship and constant encouragements have been critical to my success. I am deeply appreciative of his generosity of time and resources and of the culture he has created in the lab, which balances humour and collaboration with research excellence. I would also like to thank my lab mates, particularly Harry, Zack, Trevor and Martin, for all of their help in the lab and for making every day fun. Much gratitude and love to my parents for their encouragements and insights. Finally, I would like to thank Warren, who is the best partner I could have ever hoped for, and whose support and love mean the world to me. And Corrie, who was there for every part of my grad school experience, and whose friendship I will continue to treasure for years. 


\section{Dedication}

Je dédie cette thèse à mes grands-parents. Mimi et Grand-Père ont toujours eu l'éducation très à cœur et se sont toujours intéressés à mon parcours académique. Ils ont transmis leur amour de l'école à ma mère, qui, avec mon père, a développé ma curiosité scientifique dès mes tous premiers projets d'expo-science. Ils sont une source d'inspiration et de support inébranlable. 


\section{Table of Contents}

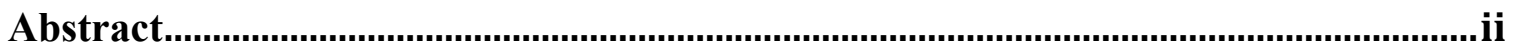

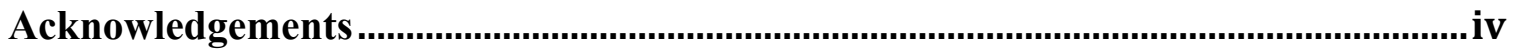

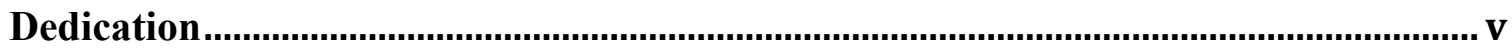

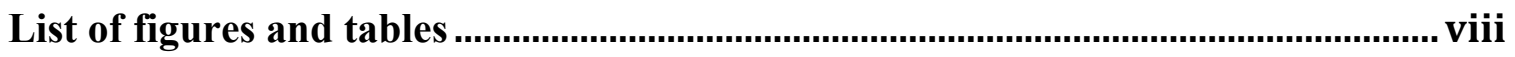

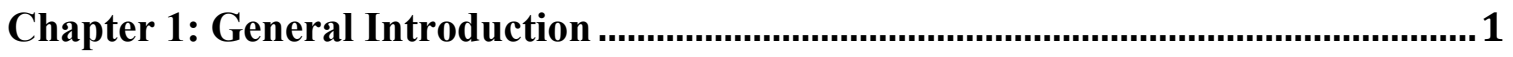

Homeostatic and non-homeostatic regulation of food intake.................................................. 2

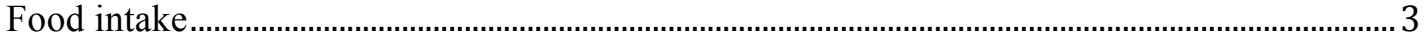

The dopaminergic meso-cortico-limbic reward system ................................................................. 5

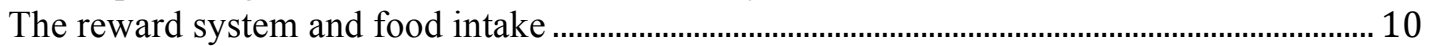

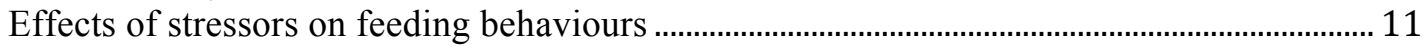

Neural integration of stress, reward \& food intake..................................................................... 12

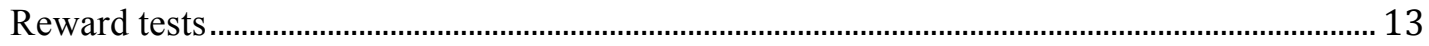

Ghrelin

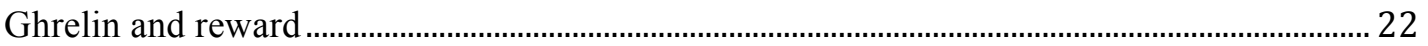

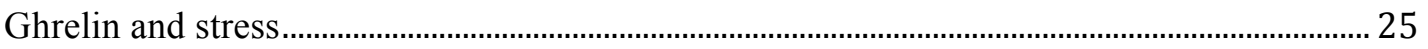

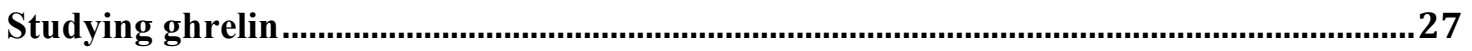

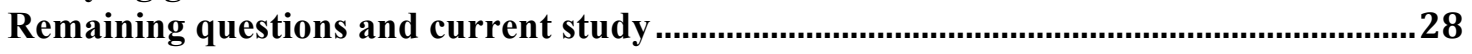

Hypothesis 1: ghrelin contributes to dessert consumption........................................................ 28

Hypothesis 2: ghrelin facilitates the reinstatement of palatable food seeking that is triggered

by food priming and food associated cues. ................................................................................... 29

Hypothesis 3: ghrelin facilitates the reinstatement to palatable food seeking that is triggered by food priming, food associated cues and stress by acting at the level of the VTA.............. 29

Chapter 2: General methods ........................................................................................... 30

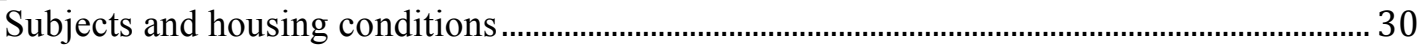

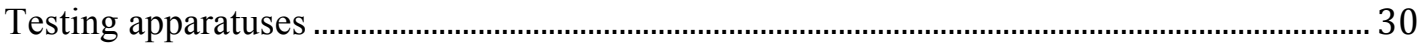

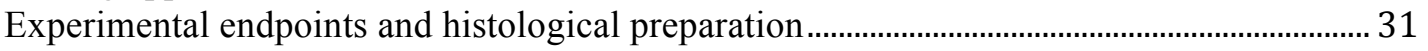

Chapter 3: The role of ghrelin in dessert consumption ................................................. 32

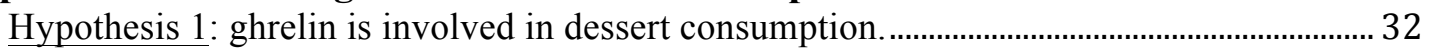

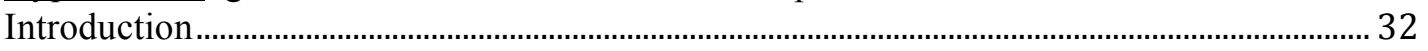

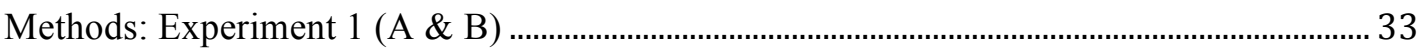

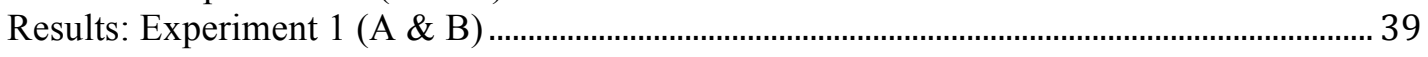

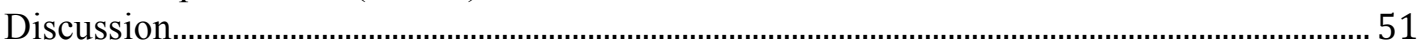

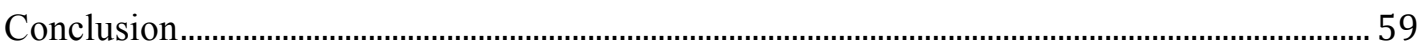

Chapter 4: The role of ghrelin in reinstatement of food seeking - operant

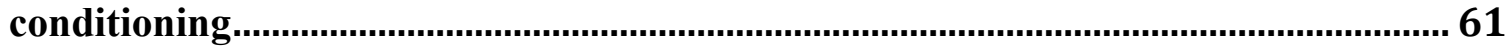

Hypothesis 2: ghrelin facilitates the reinstatement of palatable food seeking that is triggered

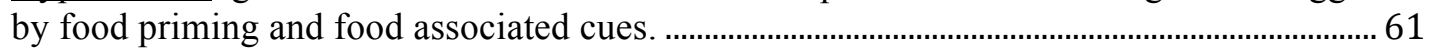

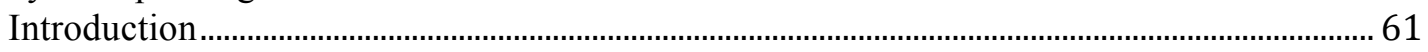

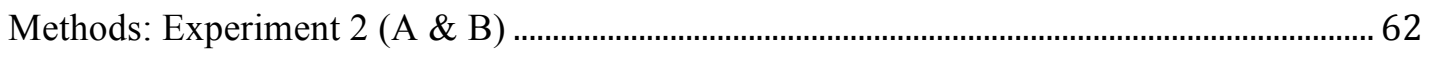

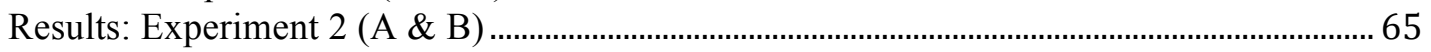

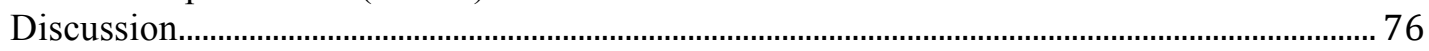

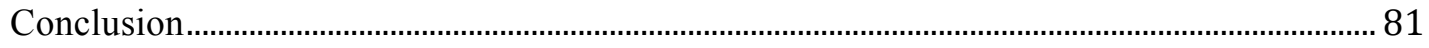


Chapter 5: The role of ghrelin in reinstatement - conditioned place preference..... 82

Hypothesis 2: ghrelin facilitates the reinstatement of palatable food seeking that is triggered

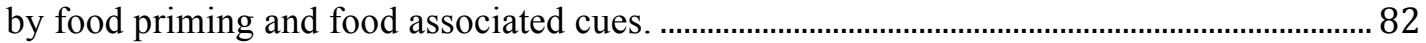

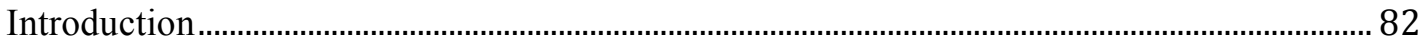

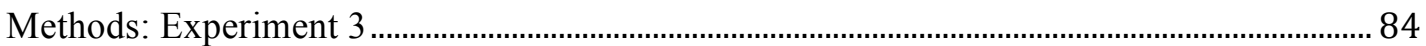

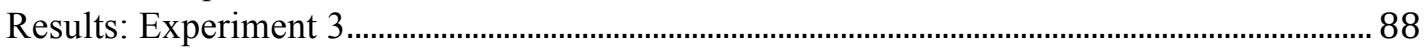

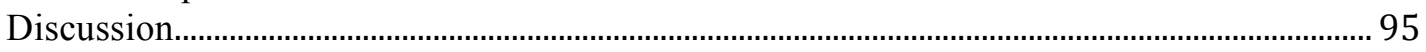

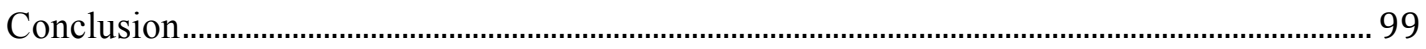

Chapter 6: The role of intra-VTA ghrelin in reinstatement ....................................100

Hypothesis 3: ghrelin facilitates the reinstatement to palatable food seeking that is triggered

by food priming, food associated cues and stress by acting at the level of the VTA............100

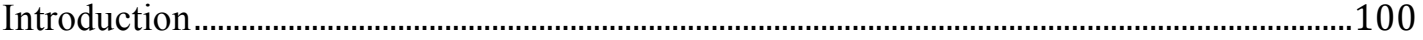

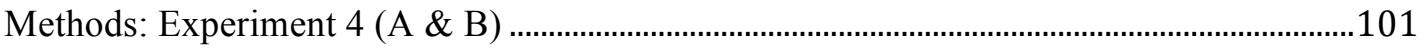

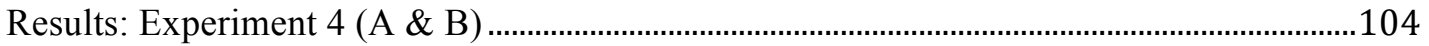

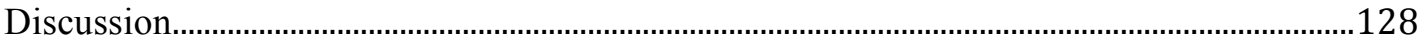

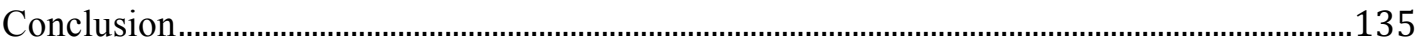

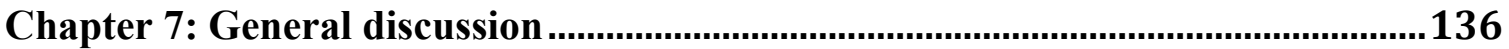

Conclusion ...................................................................................................................... 141

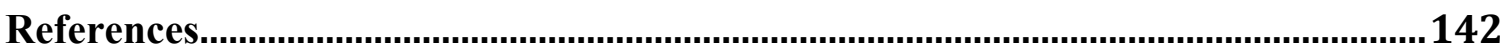




\section{List of figures and tables}

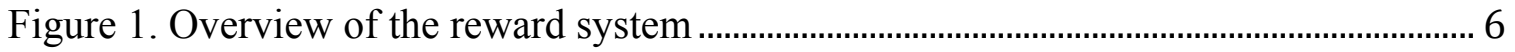

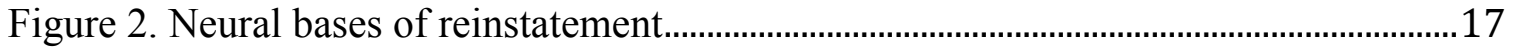

Figure 3. Locomotion in GHSR-KO and WT rats .........................................................4

Figure 4. Chow and cookie dough (CD) intake in GHSR-KO and WT rats ........................43

Figure 5. Neuronal activation in response to $\mathrm{CD}$ ingestion/anticipation..............................4

Figure 6. Representative stains for $\mathrm{c}$-fos counts ..............................................................4

Figure 7. Neuronal activation in the nucleus accumbens.....................................................50

Figure 8. Operant responding for high fat chocolate pellets in KO and WT rats ...............67

Figure 9. Operant responding for sucrose pellets in $\mathrm{KO}$ and WT rats..................................70

Figure 10. Prefrontal cortex monoamine levels in KO and WT rats......................................72

Figure 11. Amygdala monoamine levels in KO and WT rats ................................................73

Figure 12. Arcuate monoamine levels in KO and WT rats .................................................73

Figure 13. Accumbal monoamine levels in KO and WT rats................................................75

Figure 14. Timeline of CPP tests ........................................................................................87

Figure 15. Chow and cookie dough intake in KO and WT rats (Exp. 3).............................89

Figure 16. CPP in $\mathrm{KO}$ and $\mathrm{WT}$ rats................................................................................91

Figure 17. Extinction and post-extinction CPP tests ............................................................94

Figure 18. Body weights of Long Evans (LE) rats (Exp. 4A)........................................... 106

Figure 19. Lever pressing during training and extinction (Exp. 4A) .................................108

Figure 20. Cannula placements (Exp. 4A) ……………………………………………...109

Figure 21. Cannula placements (Exp. 4A): cresyl violet stain .............................................110

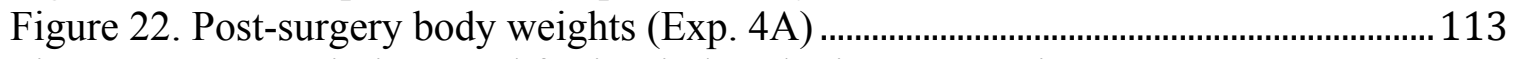

Figure 23. Cue-, priming-, and fasting-induced reinstatement in LE rats .......................... 116

Figure 24. Body weights of LE rats (Exp. 4B) ................................................................119

Figure 25. Lever pressing durign training and extinction (Exp. 4B) ................................. 121

Figure 26. Cannula placements (Exp. 4B) ……………………………………………123

Figure 27. Post-surgery body weights (Exp. 4B).............................................................. 125

Figure 28. Yohimbine-induced reinstatement in LE rats ..................................................... 127

Note: in all figures, error bars represent the standard error of the mean.

Table 1. Antibodies used for ICH in Exp. 1B...………………………………………...36

Table 2. Brain atlas coordinates for areas of interest in Exp. 1B .........................................38

Table 3. Food intake and body weight of KO and WT rats (Exp. 1B).................................45

Table 4. Body weights of grelin- and D-Lys-treated rats (Exp. 4A) .................................... 114 


\section{Chapter 1: General Introduction}

It is currently estimated that 1.4 billion adults worldwide are overweight or obese and that the incidence of this condition has nearly doubled over the last 30 years (World Health Organization, 2014). This sharp rise in incidence suggests that behavioural modifications (i.e. changes in diet and physical activity), rather than major shifts in population genetics, underlie the obesity epidemic (Nair, Adams-Deutsch, Epstein, \& Shaham, 2009). Several studies indicate a strong correlation between obesity rates and the consumption of cheap and easily obtained energy-dense foods (Bray, Nielsen, \& Popkin, 2004; Drewnowski \& Specter, 2004; Elliott, Keim, Stern, Teff, \& Havel, 2002; Ludwig, Peterson, \& Gortmaker, 2001). Paradoxically, over $80 \%$ of overweight individuals are unsuccessful in losing weight via behavioural modifications (Wing \& Phelan, 2005). Relapse to preferred foods and unhealthy eating habits is a primary concern for people undergoing weight reduction diets, especially in the face of stress or upon re-exposure to the banned foods and/or the cues associated with them (Fedoroff, Polivy, \& Herman, 1997; Gorin, Phelan, Wing, \& Hill, 2004; Greeno \& Wing, 1994; Grilo, Shiffman, \& Wing, 1989). In light of the numerous health risks associated with obesity (e.g. cardiovascular disease and diabetes, World Health Organization, 2014), the social and biological factors underlying the motivation to seek and consume energydense foods and the relapse to these foods are under intense investigation. 


\section{Homeostatic and non-homeostatic regulation of food intake}

The importance of eating for survival is reflected in the redundancy of brain structures that control various aspects of food intake, from the recognition of edible substances to the careful preparation of complex and palatable meals. These structures are widely distributed throughout the central nervous system, and form networks that are hierarchically organized.

At the most basic level, neurons within brainstem nuclei regulate food intake in a homeostatic fashion, as to maintain equilibrium between caloric intake and energy expenditure. For instance, the nucleus of the solitary tract receives direct projections from the alimentary canal and can counteract energy depletion via activation of motor systems involved in the ingestion of food, and parasympathetic activation of the digestive system (reviewed in Berthoud, 2004). Similarly, the dorsal vagal complex of the caudal brainstem can inhibit food intake upon reception of visceral signals of satiety (Smith, Jerome, \& Norgren, 1985). Thus, the brainstem harbours mechanisms that can translate food-related sensory information into adaptive behavioural motor output. This feedback system is extremely well conserved phylogenetically. Nevertheless, this is a simple system that could not have adequately responded to evolutionary pressures such as famines and other environmental threats. Thus, food intake is also regulated by a number of non-homeostatic mechanisms that allow animals to adapt their dietary behaviours to changing circumstances. Since most species have evolved in restrictive environments where the procurement of food often came at a high physical risk, these systems promote the intake of energy-dense foods by increasing their emotional valence (i.e., rewarding properties), and modulate consumption in response to stressors (Berthoud, 2004). 
It is generally accepted that the current obesity epidemic is chiefly attributable to the ability of non-homeostatic food intake mechanisms to override the homeostatic ones, as opposed to dysregulation of the latter (Berthoud, 2004). For this reason, research in this area focuses on the overlap between the neural substrates of food intake, reward and the stress response.

\section{Food intake}

The hypothalamus is a key player in the central regulation of metabolic processes, including those involved in food intake. Specifically, neurons in the arcuate nucleus, located at the base of the hypothalamus, respond to peripheral hormones that signal either a positive or negative energy balance. These neurons are responsible for the transduction of hormonal signals into neuronal ones, and can activate both orexigenic (appetite stimulating) and anorexigenic (appetite suppressing) hypothalamic pathways.

Arcuate neurons co-expressing the peptides $\alpha$-melanocyte-stimulating hormone $(\alpha \mathrm{MSH})$ and cocaine- and amphetamine-regulated transcript (CART) are the first order neurons in anorexigenic pathways, and those co-expressing neuropeptide Y (NPY) and Agouti-related peptide (AgRP) activate the orexigenic pathways. The downstream effectors of these pathways include several other hypothalamic nuclei such as the paraventricular (PVN), ventromedial (VMH), and dorsomedial (DMH) nuclei of the hypothalamus as well as the lateral hypothalamic area (LHA). The PVN is a key area in the central regulation of stress and inhibits feeding upon electrical stimulation. In rats, central and hypothalamic NPY injections result in increased food intake, presumably partly via inhibition of PVN neurons. AgRP stimulates feeding via inhibition of $\alpha \mathrm{MSH}-$ and CART-containing neurons. The VMH is an important satiety centre that is 
reciprocally connected with the arcuate nucleus. The VMH increases the activity of arcuate $\alpha \mathrm{MSH}-$ and CART-containing neurons, and lesions in this area induce hyperphagia. Finally, both populations of arcuate neurons project to the LHA, which, as opposed to the PVN, promotes food intake upon electrical stimulation (reviewed in: Gao \& Horvath, 2007; Schwartz, Woods, Porte, Seeley, \& Baskin, 2000).

Cells in the LHA and adjacent perifornical area (PFA) and DMH produce the appetite-stimulating peptides orexin-A and orexin-B. Central administration of orexin induces feeding, whereas orexin receptor (OXR1 and 2) blockade inhibits feeding (reviewed in Tsujino \& Sakurai, 2009). In addition to arcuate neurotransmitters, a number of peripherally derived energy signalling molecules converge onto orexinproducing neurons. Ultimately, low energy states increase their firing rates, which can in turn induce feeding. This orexigenic effect may be partly mediated by NPY, as reciprocal connections exist between the LHA orexin and arcuate NPY-containing neurons and intracerebroventricular orexin administration induces Fos activity in NPY neurons (Gao \& Horvath, 2007; Shioda et al., 2008; Tsujino \& Sakurai, 2009; Yamanaka et al., 2000).

Thus, like a number of brainstem nuclei, the hypothalamus regulates food intake homeostatically, a process that starts in the arcuate nucleus. However, the activity of the NPY/AgRP, $\alpha \mathrm{MSH} / \mathrm{CART}$, and orexin neurons is also modulated by hormones/neurotransmitters signalling other behavioural and physiological states (e.g. arousal, stress, pregnancy, etc...) (Berthoud, 2004), thereby adding an extra layer of flexibility in feeding behaviours that is not seen at the level of the brainstem. This flexibility is further enhanced by interactions between the hypothalamus and other non- 
homeostatic food regulation systems. This includes, but is not restricted to, the network of structures that convey emotional salience to stimuli, in other words the reward system.

\section{The dopaminergic meso-cortico-limbic reward system}

In the early 1950 s, the observation that animals will work to receive electrical stimulation in certain areas of the brain while neglecting important physiological needs such as food and water was the first indication of a reward system within the brain (Olds \& Milner, 1954). Brain stimulation studies highlighted a number of key players in the reward system. For example, rats were shown to avidly perform operant responses (such as pressing a lever) to receive stimulation in the LHA and other areas such as the prefrontal cortex (PFC), ventral tegmental area (VTA) of the midbrain, and the nucleus accumbens (NAC). Experimental manipulations of the neurotransmitter dopamine (DA) altered these responses. Soon, studies using drugs of abuse considered pleasurable in humans deepened our understanding of the now classic dopaminergic reward system (Figure 1) (reviewed in Fulton, 2010). 


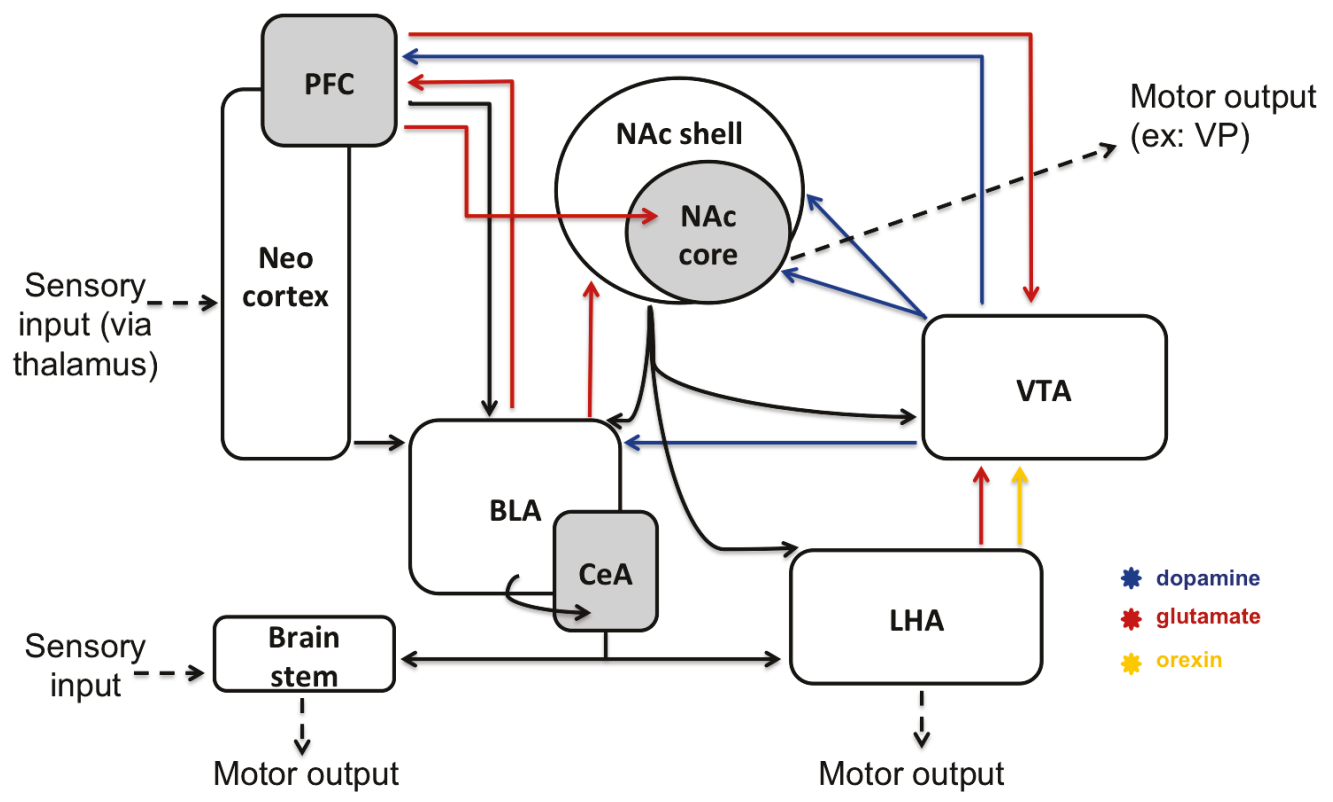

Figure 1. Overview of the meso-cortico-limbic reward system. Only connections described in the text are illustrated. PFC: prefrontal cortex; NAC: nucleus accumbens; BLA: basolateral amygdala; CeA: central amygdala; VTA: ventral tegmental area; LHA: lateral hypothalamic area. 
The DA cell bodies of the reward pathway are located in the VTA. Neuronal activity at this level is associated with reward-seeking behaviours (Borgland et al., 2009; Borgland, Malenka, \& Bonci, 2004). DA cells' burst firing, along with DA release in their target areas, increase in response to both rewarding substances and the cues that predict them (Richardson \& Gratton, 1998; Roitman, Stuber, Phillips, Wightman, \& Carelli, 2004; reviewed in: Wise, 2006). In contrast, DA depletion and DA receptor antagonists in several of these areas attenuate reward-seeking behaviours (reviewed in Berridge \& Robinson, 1998). Although the exact role of DA in reward is not fully understood, it is generally accepted that it contributes to an animal's willingness to work for rewards rather than affecting their hedonic valuation of the rewards (Berridge \& Robinson, 1998; Peciña, Cagniard, Berridge, Aldridge, \& Zhuang, 2003).

The activity of the VTA DA cells is modulated by the VTA's various inputs, which include, amongst many, glutaminergic inputs from the PFC and LHA as well as orexin projections from the LHA (Borgland et al., 2009; reviewed in Wise, 2002). These DA cells in turn send projections that reach a number of cortical and limbic areas such as the amygdala, the PFC, and the nucleus accumbens. These structures underlie all higher processes associated with reward.

The amygdala is well known for its role in associated learning and emotional processing. It consists of several nuclei that form the central amygdala (CeA) and the basolateral amygdala (BLA). In the "classic" view of amygdala function, the BLA is mostly involved in emotional classical learning; it receives sensory information from the cortex and makes associations between unconditioned (US) and conditioned (CS) stimuli (but may not be necessary for the maintenance of that link) and uses this information to 
control the activity of the CeA (Baxter \& Murray, 2002; Cardinal, Parkinson, Hall, \& Everitt, 2002). The CeA, in turn, orchestrates the behavioural, autonomic and endocrine responses to the CS via its connections with the hypothalamus and brain stem. In other words, the CeA coordinates the "affective" response to US-CS associations processed in the BLA (Cardinal et al., 2002). Recent studies however indicate a broader functional mandate for both the BLA and the CeA and it appears that amygdala nuclei work in parallel as well as serially (Cardinal et al., 2002). For instance, the BLA sends glutaminergic projections to the $\mathrm{PFC}$ and nucleus accumbens to direct complex behaviours based on learned associations. On the other hand, the CeA is also involved in the attentional aspects of the stimulus processing and the behavioural and physiological responses to stressors (reviwed in: Cardinal, Parkinson, Hall, \& Everitt, 2002; Kalivas \& Volkow, 2005; Weiss, 2005).

The BLA is reciprocally connected with the PFC (Hoover \& Vertes, 2007; Krettek \& Price, 1977; McDonald, 1987). The PFC in turn has a large influence over signalling in the nucleus accumbens (Fulton, 2010). In fact, DA innervation from the VTA to the accumbens can amplify the PFC's glutaminergic projections to the accumbens (Cardinal et al., 2002). As in humans, the rat PFC is responsible for executive functions such as attention, working memory, and decision-making (Dalley, Cardinal, \& Robbins, 2004). In the reward system, the PFC guides behaviour on the basis of the anticipated value of an action (Cardinal et al., 2002; Kalivas \& Volkow, 2005). In other words, as opposed to the amygdala, which plays a role in processing stimuli's emotional valence, the PFC determines the intensity of the behavioural response to stimuli (Kalivas \& Volkow, 2005). 
The nucleus accumbens receives glutaminergic inputs from the PFC, amygdala, and other mesocortical areas and projects to the hypothalamus (including the LHA) and motor output structures such as the ventral pallidum. Due to this central position in the reward system, the accumbens is thought to be important in the integration of the emotional, motivational, and behavioural aspects of rewards, and is often said to "turn motivation into action" (reviewed in: Fulton, 2010; Smith, Tindell, Aldridge, \& Berridge, 2009). Indeed, abundant evidence indicates that the reinforcing action of drugs is due to DA signalling in this node (Fulton, 2010). The accumbens consists of 2 major divisions that exhibit very different connectivity and functions: the accumbens' shell and core. The shell is sometimes considered part of the extended amygdala (Kalivas \& Volkow, 2005). It is highly connected to subcortical and midbrain structures such as the amygdala, LHA and VTA (Kalivas \& Volkow, 2005). DA release here is sensitive to primary reinforcers and is thought to mediate the motivational impact of US (Cardinal et al., 2002). In contrast, the core more closely resembles the dorsal striatum, a part of the extrapyramidal system that is responsible for the fine-tuning of motor functions (Cardinal et al., 2002; Weiss, 2005). DA release in the core is sensitive to CS and supports the ability of CS to drive goal directed behaviours (Cardinal et al., 2002; Fuchs, Evans, Parker, \& See, 2004; Ito, Dalley, Howes, Robbins, \& Everitt, 2000; Ito, Robbins, \& Everitt, 2004; Kalivas \& Volkow, 2005). Thus, generally speaking, the shell mediates the reinforcing effects of rewards, and the core, working together with the PFC, coordinates behavioural activation and the performance of instrumental responses for rewards. Some evidence suggests that the shell can amplify the behavioural output of the core via its connections with VTA DA neurons (Haber, Fudge, \& McFarland, 2000). 


\section{The reward system and food intake}

The dopaminergic meso-cortico-limbic system promotes a variety of appetitive behaviours and is a key contributor in the non-homeostatic regulation of food intake. Beyond our own subjective experiences, empirical evidence supports the notion that eating, particularly foods that are sweet and high in fat, is rewarding (Lowe \& Butryn, 2007). When given the choice, rats chose sugar over cocaine (Lenoir, Serre, Cantin, \& Ahmed, 2007). In the brain, a link between food intake and reward has been suspected since the discovery that electrical stimulation of the LHA induces both food intake and brain reward stimulation, and that food restriction augments reward seeking (reviewed in Fulton, 2010). Correspondingly, satiety signals from the periphery attenuate the tonic firing of VTA DA cells and inhibit LHA brain reward stimulation (Fulton, 2000; Krügel, Schraft, Kittner, Kiess, \& Illes, 2003).

Consistent with the role of the mesolimbic system in food intake, changes in DA tone affect an animal's motivation to obtain food without affecting free feeding (Fulton, 2010). Direct stimulation of the accumbens induces feeding (Kelley, Bakshi, Fleming, \& Holahan, 2000). Meanwhile, palatable foods and associated CS trigger DA release in the PFC and accumbens core (Bassareo \& Di Chiara, 1997; Richardson \& Gratton, 1998). With time, palatable foods that are consumed in excess can cause neuro-adaptations in these structures that resemble those seen after repeated drug use (Avena, Rada, \& Hoebel, 2008).

Ultimately, the expression of goal directed behaviours for food depends on the integrated activity of the reward system and other networks involved in the nonhomeostatic regulation of food intake such as those that mediate the stress response. 


\section{Effects of stressors on feeding behaviours}

Energy regulation and adequate dissemination of available resources is central to the fight or flight responses elicited by perceived threats in the environment. Accordingly, food intake must also be modulated by neuroendocrine systems responsible for instigating the stress response, including the hypothalamic-pituitary-adrenal (HPA) axis.

The activity of the HPA axis is initiated by corticotropin releasing hormone (CRH)-containing neurons in the PVN. Exposure to stressful stimuli results in the release of $\mathrm{CRH}$ from these neurons onto the pituitary gland, located at the base of the hypothalamus. Here, CRH stimulates the release of adrenocorticotrophic hormone $(\mathrm{ACTH})$ into the blood stream, eventually leading to the production/secretion of glucocorticoids (GCs) from the adrenal glands. Ultimately, GCs enhance glucose availability in the periphery and therefore provide the rapid fuel source required to meet the energetic demands of the stressor. Furthermore, GCs also act centrally as a regulatory feedback mechanism, to limit any further activity of the HPA axis (Adam \& Epel, 2007; Tsigos \& Chrousos, 2002).

Acute activation of the HPA axis prepares an organism for immediate action by increasing the heart rate, blood pressure and blood flow to the muscles and brain, while

delaying less urgent physiological processes (Sapolsky, 2004). Exposure to stressful stimuli can inhibit food intake (Sapolsky, 2004). Activation of the HPA axis via central $\mathrm{CRH}$ injections has also been shown to reduce feeding and body weight in rodents (Arase, York, Shimizu, Shargill, \& Bray, 1988; Contarino et al., 2000; Tanaka et al., 2009). Interestingly, up to $40 \%$ of people actually increase their food intake in response 
to stressors (Oliver \& Wardle, 1999). Differences in the nature/duration of stressors and individual stress reactivity may explain these seemingly inconsistent observations. For instance, Pijlman and colleagues (Pijlman, Wolterink, \& Ree, 2003) have found that while physical stressors decrease food intake in rats, chronic emotional stress promotes eating. Other studies suggest that people who secrete more GCs in response to stressors may be more vulnerable to stress-induced feeding (Epel, Lapidus, McEwen, \& Brownell, 2001) and that chronic administration of GCs increases food intake (Tataranni et al., 1996). Finally, a growing body of literature indicates that stress-induced feeding is highly correlated with food palatability (Adam \& Epel, 2007; Dallman, 2010), implying an intimate link between stress, reward and food intake at the level of the brain.

\section{Neural integration of stress, reward \& food intake}

It is becoming increasingly apparent that continuous activation of the HPA axis, as seen in chronic stress paradigms, initiates a shift towards the ingestion of more palatable foods (Connor, Jones, Conner, Mcmillan, \& Ferguson, 2008; Dallman et al., 2003; Dallman, Pecoraro, \& la Fleur, 2005; Kandiah, Yake, Jones, \& Meyer, 2006; Pecoraro, Reyes, Gomez, Bhargava, \& Dallman, 2004; Zellner et al., 2006). Evolutionarily speaking, increasing the reward value of energy dense foods in such circumstances would be beneficial to the survival of an organism as it would help ensure sufficient energy stores for upcoming challenges.

Previous studies have shown that chronically elevated GC levels facilitate dopamingeric function in the mesolimbic system (reviewed in Marinelli \& Piazza, 2002), and increase CRH mRNA expression in the central amygdala, a key structure in the chronic stress network (Shepard, Barron, \& Myers, 2000). The consumption of preferred 
foods can in turn decrease central CRH expression and the behavioural effects of stress (reviewed in Dallman et al., 2003). Thus, chronic stress can sensitize the reward system, and ingestion of rewarding foods reduces the activity of the chronic stress network.

Finally, "stress-induced food reward dependence" refers to the theory that the emotional relief associated with comfort eating reinforces appetitive behaviours in times of stress (Adam \& Epel, 2007). In light of the overabundance of palatable foods and high levels of self-reported stress in Westernized countries (Stambor, 2006), this hypothesis could help explain the current obesity epidemic. The theory further implies that palatable foods can become addictive. While the concept of food addiction is not universally accepted (Corwin \& Grigson, 2009), relapse during dieting is frequent, and food reward seeking behaviours can persist after months of abstinence, a finding that is also reported in drug addictions (Kramer, Jeffery, Forster, \& Snell, 1989; Self \& Nestler, 1998). For this reason, a number of behavioural paradigms traditionally used in the study of drug addiction have been adapted to the study of feeding behaviours.

\section{Reward tests}

The reinforcing properties of a substance can be inferred by how much of it an animal will consume given unlimited access and whether it will choose it over another option. Alternatively, reward can be inferred using markers of reward system activation such as DA overflow in the accumbens and concurrent increases in locomotor activity (e.g.; Jerlhag, Egecioglu, Dickson, \& Engel, 2010). These tasks are valuable because they are ecologically relevant and well characterized. Nevertheless, more complex tasks, often involving a learning component, are typically used to tease apart the contribution of specific brain areas to reward behaviours or the actions of pharmacological agents. These 
include non-associative learning tasks such as the locomotor sensitization paradigm. In this task, repeated exposures to a drug lead to enhanced locomotor response to each presentation of the drug. It is the result of plastic changes throughout the reward system and its effects can be seen up to a year after the administration sequence (reviewed in Steketee \& Kalivas, 2011). So far, sensitization has been observed using cocaine, amphetamines, morphine, alcohol, nicotine, and THC (Steketee \& Kalivas, 2011), but not with foods. In contrasts, food reward has been investigated using associative learning tasks such as conditioned place preference (CPP) and operant conditioning. These 2 tasks can also be used to model relapse to drugs and palatable foods.

\section{Operant conditioning and reinstatement}

The operant conditioning self-administration task is a widely used paradigm in which animals learn that pressing a lever (or performing a nose poke) results in the delivery of a reward - a form of instrumental learning. In this case, motivation is inferred from the willingness to work to obtain a reward (Richardson \& Roberts, 1996). In 1981, de Wit and Stewart published a seminal paper in which they described a variant of this paradigm that models relapse to drugs of abuse (de Wit \& Stewart, 1981). In this variant, animals are trained to self-administer a drug or food reward by pressing on a lever. Once the lever response is established, discontinuation of reward delivery upon lever pressing (i.e. forced abstinence) results in the extinction of the behaviour. The animals then receive a behavioural or pharmacological treatment and are subjected to a last extinction session. The subsequent reinstatement of the lever pressing response, even in the absence of contingent reward delivery, is considered analogous to relapse to drug or palatable food seeking in humans (Epstein, Preston, Stewart, \& Shaham, 2006; Nair, Adams- 
Deutsch, Epstein, et al., 2009). The criterion validity of this model lies in the fact that manipulations apt to induce reinstatement under these circumstances coincide with causes of relapse in humans: re-exposure to the reward, exposure to cues associated with the reward, and exposure to stressful stimuli (Elfhag \& Rössner, 2005; Ghitza et al., 2007; Ghitza, Gray, Epstein, Rice, \& Shaham, 2006; Gorin et al., 2004; Grilo et al., 1989; James et al., 2011; Kayman, Bruvold, \& Stern, 1990; Lê et al., 1998; Nair, AdamsDeutsch, Pickens, Smith, \& Shaham, 2009; Nair, Golden, \& Shaham, 2008; Richards et al., 2008).

\section{Conditioned place preference (CPP)}

The CPP task is a classical conditioning model in which the rewarding properties of a substance are inferred based on the amount of time an animal will spend in a context previously associated with the substance (reviewed in Tzschentke, 2007). While it does not measure "active" drug seeking, it does measure a form of conditioned approach (Sanchis-segura \& Spanagel, 2006). Once a preference is established, it can be extinguished via repeated exposure to that context in the absence of the reward, and subsequently reinstated using a priming dose of the reward or a stressor (Tzschentke, 2007). Whereas a number of studies show that animals form CPP to rewarding foods (Egecioglu et al., 2010; Perello et al., 2010), we are currently unaware of studies examining reinstatement of food-associated CPP.

\section{Neural bases of reinstatement}

Relatively little is known about the neural bases of the relapse phenomenon. Available data come from studies in which drugs are injected systemically or in distinct brain areas (including studies in which selected brain areas are temporarily inactivated) 
and the effectiveness of known reinstatement stimuli is assessed. The vast majority of these studies use drugs of abuse as reinforcers. Food reward reinstatement studies suggest that there is some overlap between the circuits supporting drug and food reinstatement, but that they are not completely identical (Nair, Adams-Deutsch, Epstein, et al., 2009). In both cases however, different mechanisms appear to govern cue-, reward re-exposure (priming)-, and stress-induced reinstatement (Ghitza et al., 2007, 2006; Lê et al., 1998; Nair, Adams-Deutsch, Epstein, et al., 2009; Nair et al., 2008; Steketee \& Kalivas, 2011).

Figure 2 depicts the circuits that support each type of reinstatement. Briefly, the BLA plays a large role in cue-induced, but not priming- or stress-induced drug and food seeking reinstatement (Kalivas \& McFarland, 2003; Nair, Adams-Deutsch, Epstein, et al., 2009; Shaham, Shalev, Lu, De Wit, \& Stewart, 2003; Shalev, Grimm, \& Shaham, 2002; Steketee \& Kalivas, 2011). Based on drug studies, cue-induced reinstatement is supported by connections from the VTA to the BLA and from the BLA to the PFC, as well as direct projections from the VTA to the PFC (Kalivas \& McFarland, 2003). Neuronal activity in the VTA is critical for drug-priming reinstatement, but this has not been confirmed for food-priming reinstatement (Kalivas \& McFarland, 2003; Nair, Adams-Deutsch, Epstein, et al., 2009). Based on drug studies, DA projections from the VTA to the accumbens and PFC are involved in drug priming reinstatement (Shaham et al., 2003; Steketee \& Kalivas, 2011). Finally, brain regions involved in stress-induced reinstatement include components of the extended amygdala, such as the CeA and the shell of the nucleus accumbens, as well as connections between these structures and the PFC (Fuchs et al., 2004; Kalivas \& McFarland, 2003; Steketee \& Kalivas, 2011). It should be noted that although several stressors reinstate drug induced seeking (acute food deprivation, CS 
associated with a foot shock, pharmacological stressor), yohimbine, an alpha-2 adrenoreceptor antagonist frequently used as a pharmacological stressor, is the only one that reliably reinstates food seeking (Nair, Adams-Deutsch, Epstein, et al., 2009). Neural networks supporting all types of reinstatement converge onto the PFC and the PFC's glutaminergic projections to the core of the nucleus accumbens may represent a common final output pathway for the expression of reinstatement (Kalivas \& McFarland, 2003). 


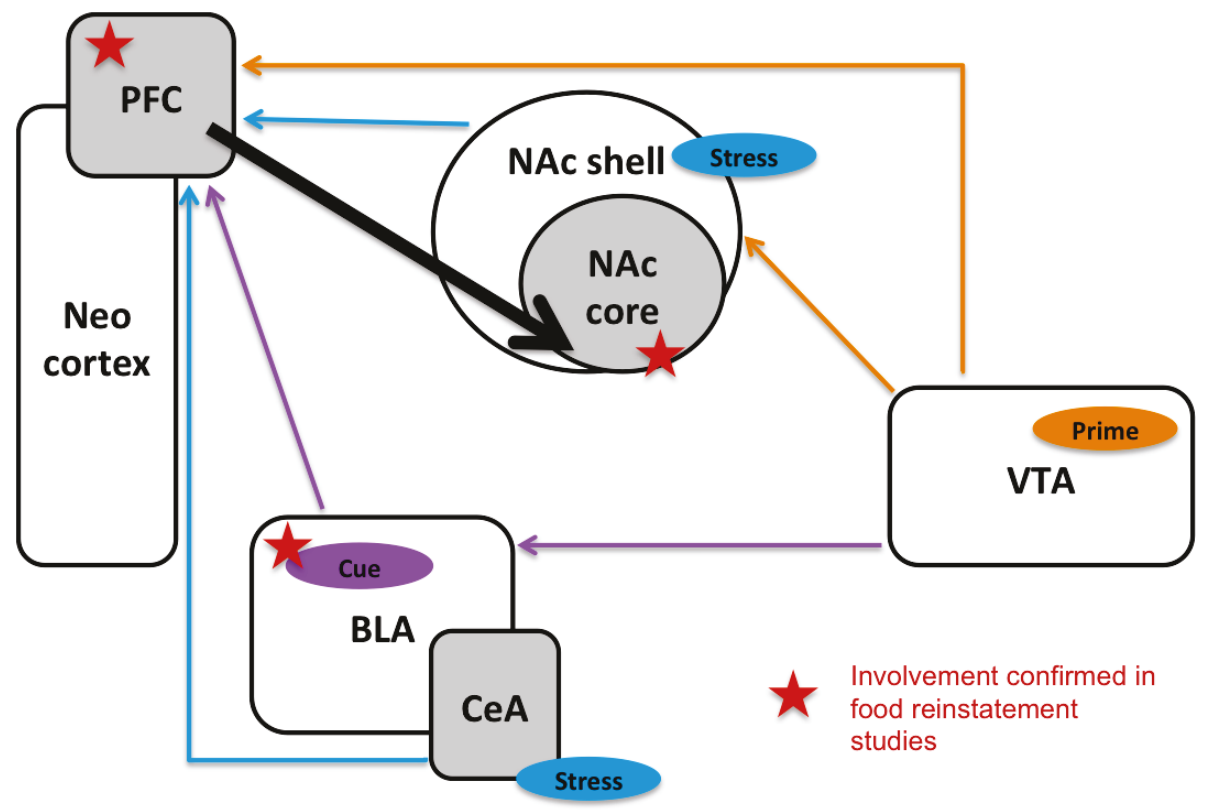

Figure 2. Overview of the neural bases of reinstatement. Only connections described in the text are shown. PFC: prefrontal cortex; NAC: nucleus accumbens; BLA: basolateral amygdala; CeA: central amygdala; VTA: ventral tegmental area. 
As with other reward behaviours, the reinstatement of drug and food seeking is affected by an animal's nutritional state and by chemical neuro-modulators that functionally link food intake and the stress response to the meso-cortico-limbic reward system (Boutrel et al., 2005; Nair, Adams-Deutsch, Epstein, et al., 2009; Nair, AdamsDeutsch, Pickens, et al., 2009; Nair et al., 2008). It is believed that manipulations of these modulators may help in the treatment of obesity by reducing reward-based overeating and preventing relapse during diet restriction (Nair, Adams-Deutsch, Epstein, et al., 2009). Currently, orexin is being investigated as a potential target for such pharmaceutical treatments (Boutrel et al., 2005; Choi, Davis, Fitzgerald, \& Benoit, 2010; Nair et al., 2008). Recent studies indicate that ghrelin, a peripheral orexigenic peptide discovered in the late 1990s, may also be an excellent candidate.

\section{Ghrelin}

Ghrelin is a 28 amino acid-long peptide that is produced predominantly in the oxyntic glands of the stomach. It is converted into its biologically active form by the post-translational addition of an octanoic acid at its third serine residue by the enzyme ghrelin-O-acyltransferase (GOAT), which is also made in the stomach (reviewed in Romero et al., 2010). Peripherally, ghrelin activates the vagus nerve, promotes gastric emptying and reduces inflammatory reactions. It is also passively transported across the blood brain barrier, and acts centrally on ghrelin receptors within the hypothalamus and other areas of the brain (Abizaid et al., 2006; Banks, Tschop, Robinson, \& Heiman, 2002; Diano et al., 2006; Guan et al., 1997; Zigman et al., 2005; reviewed in: De Smet, Mitselos, \& Depoortere, 2009; Ghigo et al., 2005; Horvath, Diano, Sotonyi, Heiman, \& Tschöp, 2001; Hosoda, Kojima, \& Kangawa, 2002). 
Ghrelin has been hypothesized to play an important role in signalling energy deficiency. Indeed, circulating ghrelin levels increase preprandially and in anticipation of a meal, and decrease postprandially (Cummings et al., 2001; Drazen, Vahl, D'Alessio, Seeley, \& Woods, 2006; Tschöp et al., 2001; Tschöp, Smiley, \& Heiman, 2000). Peripheral, intra-cranial and hypothalamic ghrelin administration produces a robust dosedependent feeding response that is not seen in animals lacking the ghrelin receptor gene (Bagnasco et al., 2003; Lawrence, Snape, Baudoin, \& Luckman, 2002; Nakazato et al., 2001; Olszewski, Grace, Billington, \& Levine, 2003; Sun, Wang, Zheng, \& Smith, 2004; Tschöp et al., 2000; Wang, Saint-Pierre, \& Taché, 2002; Wren et al., 2001). In humans, ghrelin administration has a hunger-inducing effect (reviewed in Horvath et al., 2001) and endogenous ghrelin levels are positively correlated with hunger reports (Cummings, Frayo, Marmonier, Aubert, \& Chapelot, 2004).

In the arcuate nucleus, the orexigenic effect of ghrelin is likely mediated via activation of NPY/AgRP-containing neurons (Bagnasco et al., 2003; Cowley et al., 2003; Holst \& Schwartz, 2004; Lawrence et al., 2002; Nakazato et al., 2001; Skibicka, Shirazi, Hansson, \& Dickson, 2012). The vast majority of these neurons express GHSR and exhibit c-fos activation in response to ghrelin administration (Holst \& Schwartz, 2004; Nakazato et al., 2001; Schellekens, Finger, Dinan, \& Cryan, 2012; Scott, McDade, \& Luckman, 2007). In addition, ghrelin decreases the number of excitatory inputs and increases the number of inhibitory inputs onto POMC-containing neurons cells (Pinto et al., 2004). In addition, ghrelin binding in the arcuate nucleus leads to GABA release from NPY cells, which inhibits local anorectic POMC-containing neurons (Cowley et al., 2003). Some studies suggest that ghrelin induces c-fos activation in orexin neurons and 
that this may occur independently of NPY (Toshinai et al., 2003). This might be explained by the fact that a subpopulation of arcuate neurons is presumed to produce ghrelin and innervate GHSR-expressing LHA orexin neurons (Cowley et al., 2003; Lu et al., 2002; Mondal et al., 2005), although this remains controversial (Furness et al., 2011). The disruption of orexin signalling (via either receptor antagonism or knock out of the receptor gene) reduces ghrelin-induced food intake (Perello et al., 2010; Toshinai et al., 2003).

Ghrelin's role in the conservation of energy is further demonstrated by its ability to preferentially increase the intake of fatty foods (Shimbara et al., 2004) and to reduce fat utilization while favouring oxidization of carbohydrates as a fuel source (Tschöp et al., 2000; Wortley et al., 2004). Additionally, ghrelin promotes food-seeking behaviours such as foraging and hoarding (Keen-Rhinehart \& Bartness, 2005). Its preprandial peak also coincides with the normal increase in anticipatory locomotor activity that precedes a meal (Drazen et al., 2006; Verhagen et al., 2011). This type of food anticipatory behaviour, thought to facilitate the search for food in the wild (Stephan, 2002), is enhanced by ghrelin administration in sated animals (LeSauter, Hoque, Weintraub, Pfaff, \& Silver, 2009). The effect is attenuated in GHSR-KO mice and ghrelin antagoniststreated mice and rats (Blum et al., 2009; LeSauter et al., 2009; Verhagen et al., 2011).

It is important to note that the genetic disruption of ghrelin signalling (via the knock out of ghrelin or GHSR) does not significantly alter food intake and body weight (Sun, Ahmed, Smith, \& Zivkovic, 2003; Wortley et al., 2004). Thus, it is unlikely that ghrelin is a critical regulator of energy homeostasis due to the inherent redundancy of this system. Alternatively, the presence of extra-hypothalamic ghrelin receptors in the brain 
indicates that it might be involved in the regulation of higher brain functions that are dependent on metabolic status. Based on the research conducted so far, such functions may very well include reward-based and stress-induced feeding.

\section{Ghrelin and reward}

The presence of ghrelin receptors in key nodes of the mesolimbic system strongly supports its role in reward processing. So far, GHSRs have been detected in several areas, including the VTA, amygdala, the nucleus accumbens shell, and several hypothalamic nuclei (Finger, Dinan, \& Cryan, 2012; Guan et al., 1997; Landgren, Engel, et al., 2011; Mani et al., 2014; Skibicka, Hansson, Alvarez-Crespo, Friberg, \& Dickson, 2011; Zigman, Jones, Lee, Saper, \& Elmquist, 2006). In the VTA, GHSRs are located on approximately $30 \%$ of GABAergic cells, $60 \%$ of DA cells as well as on DA cells' inputs (Abizaid et al., 2006). Ghrelin binding in this area increases the firing rates of local DA cells and DA turnover in the nucleus accumbens (Abizaid et al., 2006). Moreover, ghrelin binding in the VTA causes synaptic reorganization of local DA cells, ultimately increasing the ratio of excitatory (glutaminergic) to inhibitory (GABAergic) afferents to these cells (Abizaid et al., 2006). This results in a reduction in DA cells' firing thresholds, an effect that is not seen in GHSR-KO mice (Abizaid et al., 2006). GHSRs also form heterodimers with DA receptors in the VTA and some is target areas, which can augment the strength of DA signalling (Jiang, Betancourt, \& Smith, 2006).

Ghrelin's effects in the VTA likely account for the fact that peripheral, intracranial and intra-VTA ghrelin administration leads to behavioural outcomes that are typically associated with reward, including increased locomotion and development of CPP (Egecioglu et al., 2010; Jerlhag, 2008; Jerlhag et al., 2006, 2007; Wellman, Davis, \& 
Nation, 2005 - but see: Davis, Wellman, \& Clifford, 2007). Ghrelin's action in the VTA may also explain the fact that it can enhance the physiological and behavioural response to food rewards. For instance, ghrelin facilitates the development of CPP to palatable foods and operant responding for such foods (Finger et al., 2012; King, Isaacs, O'Farrell, \& Abizaid, 2011; Overduin, Figlewicz, Bennett-jay, Kittleson, \& Cummings, 2012; Perello et al., 2010; Skibicka et al., 2011; Skibicka, Hansson, Egecioglu, \& Dickson, 2012; Weinberg, Nicholson, \& Currie, 2011), even in sated animals (King et al., 2011; Skibicka et al., 2013). Expectedly, pharmacological and/or genetic disruption of ghrelin signalling 1) reduces the expression of CPP and operant responding for food rewards (Egecioglu et al., 2010; Finger et al., 2012; King et al., 2011; Landgren, Simms, et al., 2011; Perello et al., 2010; Skibicka et al., 2011; Skibicka, Hansson, et al., 2012), and 2) prevents increases in locomotion and accumbal DA typically observed in response to food rewards (Egecioglu et al., 2010). These effects correspond with results obtained from similar drug studies, implying that the effects of ghrelin are not limited to food rewards, but extend to other natural and artificial ones (Clifford et al., 2011; Davis et al., 2007; Jerlhag et al., 2009; Wellman et al., 2005). This is consistent with the fact that ghrelin facilitates locomotor sensitization to cocaine (Abizaid et al., 2011; Wellman, Hollas, \& Elliott, 2008) and the development of novelty place preference (Hansson et al., 2012), and that GHSR-KO rats are less sensitive to the effects of brain reward stimulation (Wellman et al., 2012).

Collectively, results from the aforementioned studies suggest that ghrelin modulates mesolimbic DA signalling as to increase the ability of rewards of all kinds to activate this system and as such, may play a role in a wide range of motivated behaviours. 
Thus, although ghrelin has an overall orexigenic effect regardless of administration route (including in the VTA - Abizaid et al., 2006; King et al., 2011; Naleid, Grace, Cummings, \& Levine, 2005; Skibicka et al., 2011), its action in the mesolimbic system may be specifically related to adding incentive value to food stimuli (Egecioglu et al., 2010; Skibicka et al., 2013). Indeed, when ghrelin is injected directly into the VTA, doses required to promote operant responding for a food reward are lower than those required to induce feeding (Skibicka et al., 2011). In a study performed by Egecioglu et al. (2010), intra-VTA ghrelin administration increased how much peanut butter rats ate, but did not affect chow intake (Egecioglu et al., 2010). When compared to sham-operated rats, VTAlesioned rats consumed less peanut butter upon i.c.v. ghrelin administration; both groups however consumed the same amount of chow after ghrelin treatment (Egecioglu et al., 2010). Ghrelin-treated VTA-lesioned rats also spent less time exploring an eppendorf tube containing peanut butter than sham-operated rats (Egecioglu et al., 2010). Blockage of DA receptors in the nucleus accumbens abolishes intra-VTA ghrelin-induced food reward behaviour (operant responding for sucrose), but not intra-VTA ghrelin-induced food intake (Skibicka et al., 2013). Together with the observation that ghrelin does not increase some reward behaviours when injected directly into the hypothalamus (Schneider, Rada, Darby, Leibowitz, \& Hoebel, 2007), these findings suggest a dissociation between ghrelin's role in energy homeostasis at the level of the hypothalamus and its role in the reward system. Ghrelin's effects in the VTA are likely independent of NPY signalling, suggesting different mechanisms of actions in these 2 areas (Naleid et al., 2005). Meanwhile, orexin appears to be necessary for some ghrelin- 
induced reward seeking behaviours (Perello et al., 2010), but not others (Jerlhag, Egecioglu, Dickson, \& Engel, 2011).

\section{Ghrelin and stress}

In addition to its role in reward, ghrelin is also involved in the activity of the HPA axis. Rises in plasma ghrelin levels occur following both acute and chronic stress stimulation (Asakawa et al., 2001; Kristenssson et al., 2006; Rouach et al., 2007), and ghrelin itself contributes to the physiological response to stress. Ghrelin alters metabolism as to favour the utilization of carbohydrates while preserving fat stores (Currie et al., 2011; Tschöp et al., 2000), which facilitates rapid defensive behaviours in times of stress (Depke et al., 2008). Moreover, ghrelin contributes to stress-induced eating and has important implications for psychiatric conditions exacerbated by stress such as anxiety and depression.

Ample evidence suggests that GCs and ghrelin work together in the modulation of food intake in response to stress. For instance, inhibition of GCs through adrenalectomy abolishes ghrelin-induced feeding (Spinedi et al., 2006). Interestingly, GHSR-KO mice are resistant to the anorexic effects of chronic stress exposure even though they demonstrate GCs rises equivalent to wild type (WT) stressed animals (Patterson, Ducharme, Anisman, \& Abizaid, 2010). The relationship between ghrelin and HPA axis derivatives likely occurs via ascending monoaminergic pathways (e.g. via ghrelin receptor-expressing noradrenalin projections to the hypothalamus) (Patterson et al., 2010), and directly at the hypothalamic level. The latter possibility is supported by the fact that ghrelin-containing arcuate neurons project to CRH cells in the PVN (Cowley et al., 2003), and ghrelin administration results in the production of CRH as well as elevated 
circulating ACTH and GC levels (Arvat et al., 2001; Asakawa et al., 2001; Tassone et al., 2003).

The downstream effects of ghrelin-mediated HPA axis activation are currently the subject of considerable debate. Several studies report that ghrelin administration induces anxiety- and depressive-like behaviours that can be reversed by inhibition of CRH signalling (Asakawa et al., 2001; Carlini et al., 2002; Carvajal, Carlini, Schiöth, de Barioglio, \& Salvatierra, 2009; Currie et al., 2012). Other studies report opposite effects (Lutter et al., 2008). In a recent review article, Chuang and Zigman (2010) propose that such conflicting results may reflect the fact that stress-induced ghrelin elevations are part of a compensatory mechanism that diminishes but does not abolish the development of anxiety and depressive symptoms (Chuang \& Zigman, 2010). Alternatively, it is possible that the effects of ghrelin on the stress response and subsequent behavioural patterns vary as a function of an individual's stress background. Indeed, rat strains that tend to be more anxious exhibit lower basal ghrelin levels and lower stress-induced ghrelin elevations than less anxious rat strains (Kristensson, Sundqvist, Håkanson, \& Lindström, 2007; Kristenssson et al., 2006). Similarly, individuals who show high GC reactivity to stressors also exhibit enhanced stress-induced food intake and ghrelin responses relative to individuals who do not (Epel et al., 2001; Rouach et al., 2007).

Interestingly, it has been suggested that the activity of the meso-limbic system is involved in mood regulation and depression (Nestler \& Carlezon, 2006), raising the possibility that the effects of ghrelin in response to stress are linked to its role in reward. Consistent with this, whereas WT mice are more likely to develop a CPP to high fat foods in response to chronic stressors, GHSR-KO mice do not (Chuang et al., 2011). In 
this paradigm, mice expressing GHSR only in DA cells were comparable to WT mice (Chuang et al., 2011). This suggests that ghrelin plays a role in "comfort eating" via modulation of DA signalling.

\section{Studying ghrelin}

A number of techniques have been developed to study the effects of ghrelin signalling. As can be seen in previous sections, these include pharmacological actions at the GHSR and genetic manipulations of ghrelin, GHSR, and GOAT. In the past, genetic approaches typically used mice, as rat embryonic cells, which are needed for the creation of knock out models, are difficult to isolate and culture (Abbott, 2004; Zan et al., 2003). Nevertheless, there are many advantages to using rat models - including the fact that rats acquire operant behaviours more readily than mice (Abbott, 2004) - and approximately 10 years ago, a number of research groups advocated for the development of KO rat models (Abbott, 2004; Jacob, 1999). Recently a GHSR-KO strain was developed at the Medical College of Wisconsin via the introduction of a point mutation in the GHSR gene of Fawn Hooded Hypertensive (FHH) rats (PhysGen Program in Genomic Application, http://pga.mcw.edu). As expected, GHSR-KO rats do not overeat in response to systemic ghrelin injections (Bülbül et al., 2011). Although this model is relatively new, studies show that compared to WT rats, GHSR-KO rats are less sensitive to the effects of cocaine as well as brain reward stimulation (Clifford et al., 2012; Wellman et al., 2012). These rats may therefore also prove useful in the study of reward-based feeding behaviours. 


\section{Remaining questions and current study}

An impressive body of literature describes the actions of ghrelin in several interrelated processes relevant to obesity. Yet, our currently incomplete understanding of its mechanisms of action hinders the development of effective pharmaceutical treatments for the prevention and treatment of this condition. The overarching goal of the present thesis is to extend findings from previous studies showing a role of ghrelin in reward-based feeding and to identify potential sites where ghrelin exerts such effects.

As mentioned earlier, ghrelin preferentially increases palatable food intake and increases the motivation to work for food in sated animals (King et al., 2011; Skibicka et al., 2013). Although this is reminiscent of studies in which individuals eat palatable desserts after filling meals, there is a paucity of studies directly linking ghrelin to dessert consumption. Similarly, very few studies have examined the role of ghrelin in relapse to rewarding substances (Maric, Sedki, Ronfard, Chafetz, \& Shalev, 2012; Tessari et al., 2007). None of those studies focused on relapse to energy-dense foods, with or without the presence of a stressor. Given that this is a major problem reported by people undergoing weight reduction diets, this gap in the literature is surprising. The present thesis examined these issues by comparing GHSR-KO rats to rats possessing an identical genetic background, with an intact GHSR gene (WT rats), as well as Long Evans rats treated with ghrelin or a GHSR antagonist. Specifically, the hypothesis and aims of this study are:

\section{Hypothesis 1: ghrelin contributes to dessert consumption.}

- Aim 1: to compare GHSR-KO and WT rats in a behavioural paradigm designed to model the dessert phenomenon. 
○ Aim 2: to determine which brain areas are activated by the ingestion and anticipation of dessert in GHSR-KO and WT rats.

Hypothesis 2: ghrelin facilitates the reinstatement of palatable food seeking that is triggered by food priming and food associated cues.

○ Aim 3: to compare GHSR-KO and WT rats in the operant conditioning version of the reinstatement test.

- Aim 4: to compare GHSR-KO and WT rats in the conditioned place preference version of the reinstatement test.

Hypothesis 3: ghrelin facilitates the reinstatement to palatable food seeking that is triggered by food priming, food associated cues and stress by acting at the level of the VTA.

○ Aim 5: to assess operant conditioning reinstatement in Long Evans rats receiving either ghrelin or a GHSR antagonist delivered chronically into the VTA. 


\section{Chapter 2: General methods}

\section{Subjects and housing conditions}

Male Long Evans rats (LE) (216 to 381 grams) and male GHSR-KO and WT Fawn Hooded Hypertensive (FHH) (179 to 479 grams) rats were used for the experiments. LE rats were obtained from Charles River and acclimated to the housing facility at Carleton University (Ottawa) for a minimum of 7 days before testing. Breeding pairs of GHSR-KO rats were obtained from the Medical College of Wisconsin and moved to our breeding colony within the Carleton University housing facility in January 2011.

All rats used in the following experiments were single-housed. Unless otherwise specified, the rats received adlib regular rat chow and tap water and were maintained on a 12 hours light/dark cycle (lights on at 8:00 AM). All procedures were conducted in accordance with the Canadian Council of Animal Care (CCAC) guidelines and were approved by the Animal Care Committee at Carleton University.

\section{Testing apparatuses}

The following testing apparatuses were used in this study: infrared-operated activity monitors $(\mathrm{n}=20$; Micromax, Accuscan Instruments), operant conditioning chambers $(\mathrm{n}=12$; Coulbourn Instruments), and conditioned place preference (CPP) boxes $(n=6)$.

Each operant conditioning chambers (width $\mathrm{x}$ depth $\mathrm{x}$ height $=12 \times 10 \times 13$ inches) contained a grid floor, house light, pellet delivery magazine, and 2 levers $2.5 \mathrm{~cm}$ off the floor - one selected as "active" and the other as "inactive". Three cue lights (red, yellow 
and green) were located directly above each lever. Active and inactive lever presses were recorded by Graphic State software (version 3.0). Under specific circumstances, active lever presses resulted in the delivery of a high fat chocolate-flavoured (BioServ, 45mg, $35 \%$ fat, F05879) or sucrose (BioServ, 45mg, F0042) pellet, as well as the presentation of a brief tone and illumination of the cue lights above the active lever (tone/light stimulus, 2 seconds). Inactive lever presses had no programmed consequences.

CPP boxes consisted of a white (12x12x14 inches) chamber with a plastic floor and a black chamber (same dimensions) with a metallic grid floor, connected by a smaller (3x4x14 inches) grey compartment. Guillotine doors separated each compartment.

\section{Experimental endpoints and histological preparation}

Unless otherwise specified, rats were euthanized using sodium pentobarbital ( $90 \mathrm{mg} / \mathrm{mg}$, i.p.) and perfused with $0.9 \%$ saline solution followed by a $4 \%$ paraformaldehyde solution. Where relevant, brains were extracted and stored in a $4 \%$ paraformaldehyde solution for 3 days prior to being transferred to a $30 \%$ sucrose solution. The brains were then sectioned at 60 micron intervals using a cryostat, and stored in Watson's cryo-protectant solution at -20 degrees Celsius until processed for immunohistochemistry or stained with Cresyl violet. 


\section{Chapter 3: The role of ghrelin in dessert consumption}

Hypothesis 1: ghrelin is involved in dessert consumption.

Aim 1: to compare GHSR-KO and WT rats in a behavioural paradigm designed to model the dessert phenomenon.

Aim 2: to determine which areas are activated by the ingestion and anticipation of dessert in GHSR-KO and WT rats.

\section{Introduction}

Combined with an increasingly sedentary lifestyle, overconsumption of energydense foods (i.e. reward-based consumption that exceeds metabolic need) may be partly responsible for the current obesity epidemic. Such overconsumption includes the tendency to eat palatable desserts after filling meals that effectively satisfy an individual's energy requirements and hunger sensations. Although the neural basis of the dessert phenomenon has not been fully characterized, recent studies suggest the involvement of orexin A (Choi et al., 2010). As with orexin, several lines of evidence suggest a role for ghrelin in dessert consumption.

As reviewed in chapter 1, ghrelin induces hunger in humans (reviewed in Horvath, Diano, Sotonyi, Heiman, \& Tschöp, 2001) and feeding responses in rodents (King et al., 2011; Olszewski et al., 2003; Sun et al., 2004; Tschöp et al., 2000; Zigman et al., 2005). Ghrelin also acts on GHSRs within the mesolimbic system, and particularly in dopaminergic cells of the VTA (Abizaid et al., 2006; Guan et al., 1997; Zigman et al., 2006). Correspondingly, ghrelin facilitates the expression of several reward-related behaviors (reviewed in Perelló \& Zigman, 2012). Finally, the effects of ghrelin extend to 
motivated appetitive behaviors such as foraging and hoarding (Keen-Rhinehart \& Bartness, 2005) and pre-prandial increases in endogenous ghrelin levels are related to food anticipatory activity (Drazen, Vahl, D’Alessio, Seeley, \& Woods, 2006; Verhagen et al., 2011).

The current study's first experiment was performed to test the hypothesis that beyond ghrelin's role as an interoceptive hunger signal (Davidson et al., 2005), its effects on reward processing and motivated behaviour can lead calorically sated animals to behave like hungry ones. To this end, we compared GHSR-KO and WT rats in a task designed to model the dessert phenomenon. We also compared the strains' food anticipatory activity before scheduled meals. In a follow-up experiment, we examined cfos activation in a number of brain areas and cell populations relevant to reward processing in rats that underwent the dessert task. Since factors other than dessert consumption may induce c-fos activation in those regions (ex: dessert anticipation, scheduled feeding, experimental endpoint procedures), we compared these rats' neuronal activation to that of GHSR-KO and WT rats that were exposed to a dessert after a meal but could not ingest it, as well as GHSR-KO and WT rats that ate a meal but were not exposed to a dessert.

\section{Methods: Experiment 1 (A \& B)}

Experiment 1A: Food restriction regimen, food anticipatory activity and dessert test

Nineteen rats $(9 \mathrm{KO}+10 \mathrm{WT})$ were used in Experiment $1 \mathrm{~A}$. Their cages were placed inside activity monitors and their hourly locomotor activity was recorded for the duration of the experiment. At the outset of the experiment, rats were given 4 grams of 
cookie dough (Pillsbury, chocolate chip) in a circular ceramic plate $(8 \mathrm{~cm}$ diameter) overnight to prevent future neophobic effects. Baseline food intake and locomotion were recorded for 4 days. This was followed by a 23 -day scheduled-meal period during which regular chow was only accessible from 10:00 AM to 2:00 PM (locomotion and food intake recorded daily). Testing took place on the last day of the food restriction regimen. Rats were given 30 grams of cookie dough in a ceramic plate during the last hour of the meal (i.e. from 1:00PM to 2:00PM). The amount of cookie dough eaten during this period was recorded. All rats were weighed and euthanized at the end of the meal (2:00 PM).

Experiment 1B: Immunohistochemistry (ICH) and stereology

Twenty additional rats $(10 \mathrm{KO}+10 \mathrm{WT})$ were used in Experiment $1 \mathrm{~B}$. As in Experiment 1, rats were initially given 4 grams of cookie dough overnight to prevent neophobia. During the first 10 minutes of cookie dough exposure, the ceramic plates were covered by a stainless steel sieve that allowed the rats to see and smell the cookie dough, but prevented them from eating it. Baseline monitoring and scheduled feeding proceeded as described for Experiment 1A, with the exception that rats were weighed daily (prior to the start of the meal). On the last day of testing, half of the rats (5 $\mathrm{KO}$ and 5 WT) were exposed to 30 grams of cookie dough during the last 10 minutes of the meal, but were prevented from eating it by a stainless steel sieve covering the ceramic plate containing the treat. The rats were then weighed and euthanized. The remaining rats were also euthanized at the end of the last meal but were not previously exposed to cookie dough. 
Selected brain sections (every $4^{\text {th }}$ section containing the area of interest) from rats in Experiment $1 \mathrm{~A}$ and $1 \mathrm{~B}$ were washed in $0.1 \mathrm{M}$ phosphate buffer 5 times prior to singleand double-staining ICH. Table 1 describes the primary and secondary antibodies used in each area. Briefly, sections were washed in in 1\% hydrogen peroxide (30 minutes) and blocked in bovine serum albumin with $0.3 \%$ Triton-X. Each primary and secondary antibody incubated for 2 days at 4 degrees Celsius. Sections were then incubated in avidin-biotin-HRP complex (1:200 in PB; Vector Elite Kit; Vectastain) for 60 minutes at room temperature. Incubations were followed by 35 -minute rinses in $0.1 \mathrm{M}$ PB. The sections were then immersed in $0.1 \mathrm{M}$ PB containing $0.05 \%$ 3,3'-diaminobenzidine (DAB) and $0.01 \%$ hydrogen peroxide for 30 minutes at room temperature. In order to visualize c-fos in double-stained sections, $3.5 \%$ nickel ammonium sulfate was added to the DAB solution. The reaction was stopped by three 5-minutes rinses in $0.1 \mathrm{M} \mathrm{PB}$. Sections were mounted on gelatin-covered slides, dehydrated in a graded alcohol series, rinsed in clearene and coverslipped with permount (Fisher, SP-15-100) for stereology. 
Table 1. Primary and secondary antibodies used in Experiment 1B

\begin{tabular}{|c|c|c|c|}
\hline Area & Stain & Primary Ab & Secondary Ab \\
\hline $\begin{array}{l}\text { Nucleus } \\
\text { Accumbens }\end{array}$ & $\begin{array}{l}\text { Single: } \\
\text { c-fos }\end{array}$ & $\begin{array}{l}\text { Anti-c-fos rabbit } \\
\text { (Millipore PC38, 1:20 000) }\end{array}$ & $\begin{array}{l}\text { Anti-rabbit } \\
\text { (Jackson Immunoresearch } \\
\text { 711-065-152, 1:250) }\end{array}$ \\
\hline $\begin{array}{l}\text { Arcuate } \\
\text { nucleus }\end{array}$ & $\begin{array}{l}\text { Single: } \\
\text { c-fos }\end{array}$ & $\begin{array}{l}\text { Anti-c-fos rabbit } \\
\text { (Millipore AB-5, 1:20 000) }\end{array}$ & $\begin{array}{l}\text { Anti-rabbit } \\
\text { (Jackson Immunoresearch } \\
711-0650152,1: 250)\end{array}$ \\
\hline $\begin{array}{l}\text { Amygdala } \\
\text { (BLA \& CeA) }\end{array}$ & $\begin{array}{l}\text { Single: } \\
\text { c-fos }\end{array}$ & $\begin{array}{l}\text { Anti-c-fos rabbit } \\
\text { (Millipore PC38, 1:20 000) }\end{array}$ & $\begin{array}{l}\text { Anti-rabbit } \\
\text { (Jackson Immunoresearch } \\
\text { 711-0650152, 1:250) }\end{array}$ \\
\hline \multirow{4}{*}{$\begin{array}{l}\text { PFA and } \\
\text { LHA }\end{array}$} & \multirow{2}{*}{$\begin{array}{l}\text { Double: } \\
\text { orexin-B } \\
\text { and c-fos }\end{array}$} & $\begin{array}{l}\text { Anti-Orexin B goat } \\
\text { (Santa Cruz Biotechnology } \\
\text { C-19 sc- } 8071,1: 10000 \text { ) }\end{array}$ & $\begin{array}{l}\text { Anti-goat } \\
\text { (Jackson Immunoresearch } \\
705-065-147,1: 250)\end{array}$ \\
\hline & & $\begin{array}{l}\text { Anti-c-fos rabbit (Millipore } \\
\text { PC } 38,1: 20 \text { 000) }\end{array}$ & $\begin{array}{l}\text { Anti-rabbit } \\
\text { (Jackson Immunoresearch } \\
\text { 711-0650152, 1:250) }\end{array}$ \\
\hline & \multirow{2}{*}{$\begin{array}{l}\text { Double: } \\
\text { orexin-A } \\
\text { and c-fos }\end{array}$} & $\begin{array}{l}\text { Anti-Orexin A goat } \\
\text { (Santa Cruz Biotechnology } \\
\text { sc- } 8070,1: 5000)\end{array}$ & $\begin{array}{l}\text { Anti-goat } \\
\text { (Santa Cruz Biotechnology } \\
\text { sc-2020, 1: 250) }\end{array}$ \\
\hline & & $\begin{array}{l}\text { Anti-c-fos rabbit } \\
\text { (Millipore PC38, 1:20 000) }\end{array}$ & $\begin{array}{l}\text { Anti-rabbit } \\
\text { (Jackson Immunoresearch } \\
711-0650152,1: 250)\end{array}$ \\
\hline \multirow{2}{*}{ VTA } & \multirow{2}{*}{$\begin{array}{l}\text { Double: } \\
\text { tyrosine } \\
\text { hydroxylase } \\
\text { and c-fos }\end{array}$} & $\begin{array}{l}\text { Anti-Tyrosine Hydroxylase } \\
\text { sheep (Millipore AB-1542, } \\
1: 10000)\end{array}$ & $\begin{array}{l}\text { Anti-sheep } \\
\text { (Jackson Immunoresearch } \\
\text { 713-065-003, 1: 250) }\end{array}$ \\
\hline & & $\begin{array}{l}\text { Anti-c-fos rabbit } \\
\text { (Millipore AB-5, 1:20 000) }\end{array}$ & $\begin{array}{l}\text { Anti-rabbit } \\
\text { (Jackson Immunoresearch } \\
711-0650152,1: 250)\end{array}$ \\
\hline
\end{tabular}


Each area of interest was outlined according to (Paxinos \& Watson, 1998) at 4x magnification (Table 2). The orexin cell field was divided into the PFA and LHA according to Harris, Wimmer, \& Aston-Jones, 2005. Cell counts were performed at 60x magnification with immersion oil. Using the Stereo Investigator's optical fractionator method, systematic random sampling was performed within each area of interest to obtain an estimate of the total number of labeled cells. 
Table 2. Brain atlas coordinates (Paxinos \& Watson, 1998) used to outline areas of interest in Experiment 1B (in mm from landmark)

\begin{tabular}{lccc} 
& $\begin{array}{c}\text { Anterior-posterior } \\
\text { axis }\end{array}$ & $\begin{array}{c}\text { Medial-lateral } \\
\text { axis }\end{array}$ & $\begin{array}{c}\text { Dorso-ventral } \\
\text { axis }\end{array}$ \\
\hline Landmark used & Bregma & Midline & $\begin{array}{c}\text { Below the surface } \\
\text { of the brain }\end{array}$ \\
\hline Accumbens - shell & +1.0 & $0.5-1.2$ & $6.0-8.0$ \\
Accumbens - core & +1.0 & $1.2-2.6$ & $6.0-8.0$ \\
PFA & -2.8 & $1.0-1.5$ & $8.5-9.4$ \\
LHA & -2.8 & $1.5-2.5$ & $8.4-9.4$ \\
Arcuate & -2.8 & $0.0-0.1$ & $9.4-10.4$ \\
BLA & -2.8 & $4.4-5.2$ & $8.0-9.0$ \\
CeA & -2.8 & $3.8-4.5$ & $7.6-8.4$ \\
VTA & -6.04 & $0.2-0.8$ & $8.0-9.0$ \\
\hline
\end{tabular}

* positive numbers are anterior to bregma and negative numbers are posterior to bregma 


\section{Results: Experiment 1 (A \& B)}

\section{Experiment 1A:}

Locomotion: Locomotion data from a total of 4 rats $(2 \mathrm{KO}+2 \mathrm{WT})$ were dropped due to technical problems with the activity monitors. A repeated measure ANOVA was performed to examine average hourly locomotion in the remaining GHSR-KO and WT rats during three days of baseline (data from the first baseline day were dropped from the analysis because rats' activity increased in a non-representative way upon being moved to micromax chambers). A significant main effect of strain indicated that $\mathrm{KO}$ rats are hypoactive relative to WT rats $[\mathrm{F}(1,13)=17.976, \mathrm{p}=0.001]$. The analysis also revealed a significant main effect of hour $[\mathrm{F}(23,299)=45.724, \mathrm{p}<0.001]$ and a significant strain by hour interaction effect $[\mathrm{F}(23,299)=8.096, \mathrm{p}<0.001]$. Visual inspection of the data indicated that rats were more active during the dark phase, and this effect was more pronounced in WT rats than in KO rats (Figure 3A).

A repeated measure ANOVA looking at average hourly locomotion of $\mathrm{KO}$ and WT rats during 23 days of food restriction revealed similar but less pronounced effects as during the baseline period. Under these conditions, $\mathrm{KO}$ rats were still hypoactive relative to $\mathrm{WT}$ rats $[\mathrm{F}(1,13)=10.241, \mathrm{p}=0.007]$ and all rats were still more active during the dark phase of the day $[\mathrm{F}(23,299)=51.892, \mathrm{p}<0.001]$, but there was no strain by hour interaction $[\mathrm{F}(23,299)=1.430, \mathrm{p}=0.094]($ Figure $3 \mathrm{C})$.

Food anticipatory activity: Locomotion during the hour preceding the start of the meal was examined as a measure of food anticipatory activity. As expected, a repeated measure ANOVA revealed that rats increased their activity during this period over the course of the food restriction regimen $[\mathrm{F}(22,286)=5.791, \mathrm{p}<0.001]$. Interestingly, WT 
rats moved more during this period than $\mathrm{KO}$ rats $[\mathrm{F}(1,13)=9.772, \mathrm{p}=0.008]$ despite having similar scores at that time during the baseline period $[\mathrm{t}(13)=0.981, \mathrm{p}=0.345]$. Finally, there was no strain by day interaction in locomotor activity prior to meals $[F(22,286)=0.874, p=0.629]($ Figure $3 B, C$ and $D)$. 

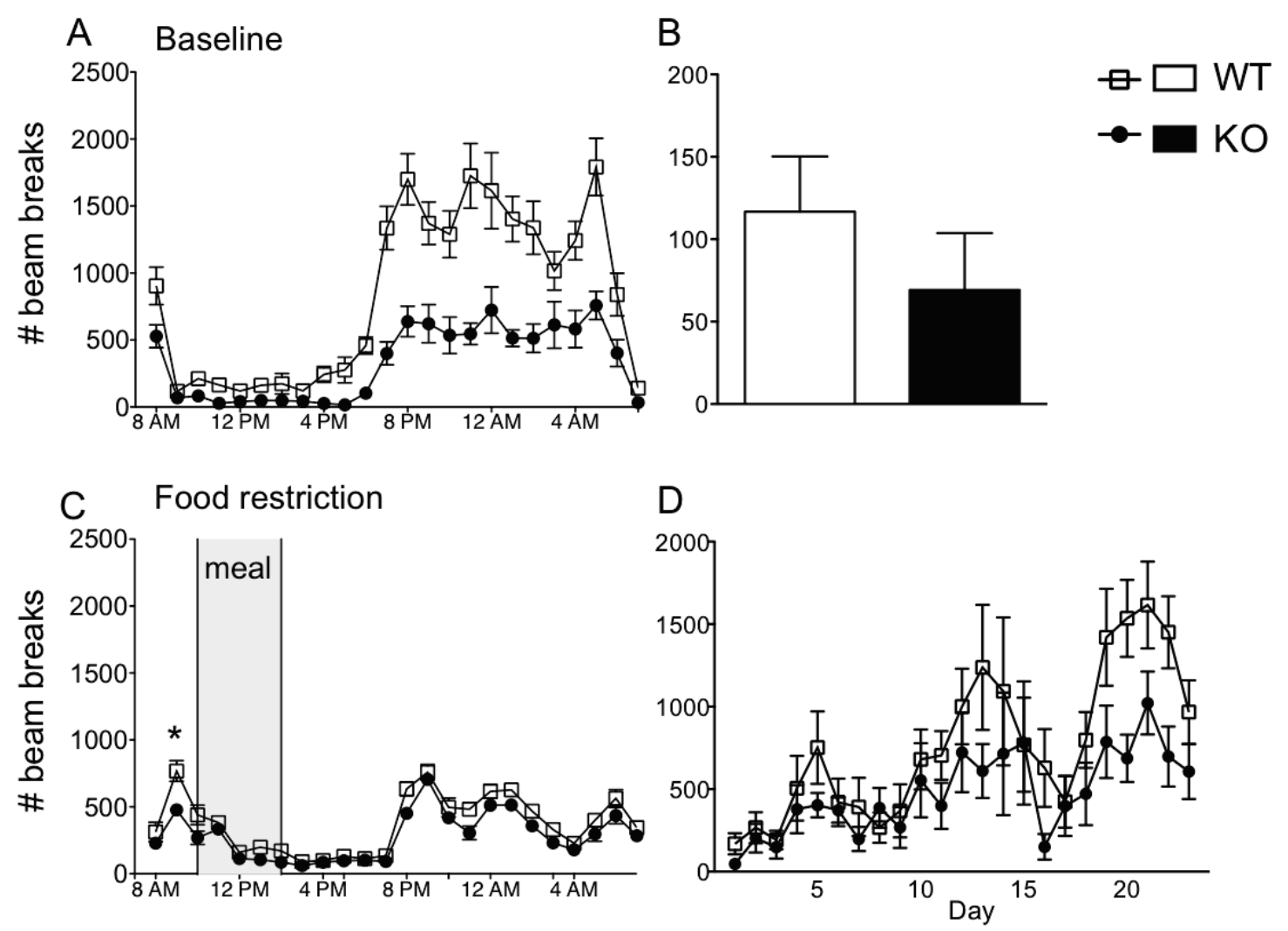

Figure 3. Locomotion in GHSR-KO $(n=7)$ and WT rats $(n=8)$ in Experiment 1 A. A. Hourly locomotion during the baseline period (average of 3 days). B. Locomotion between 9 and 10 am during baseline (average of 3 days) C. Hourly locomotion during the food restriction period (average of 23 days). D. Locomotion between 9 and 10 am during 23 days of food restriction. 
Chow and cookie dough intake: GHSR-KO and WT rats consumed similar amounts of chow during baseline and food restriction (baseline (average of 3 days): [t(17) $=-0.738, p=0.4703]$; food restriction: $[F(1,17)=0.050, p=0.825])$. All rats increased their food intake throughout the course of the food restriction regimen $[F(22,374)=$ 10.111, $\mathrm{p}<0.001]$. They were also indistinguishable in terms of chow intake (absolute food intake: $[\mathrm{t}(17)=-0.563, \mathrm{p}=0.581$; food intake per grams of body weight: $[\mathrm{t}(17)=-$ 1.054, $\mathrm{p}=0.307])$ and weight $[\mathrm{t}(17)=0.721, \mathrm{p}=0.480]$ on the day of testing. On average, GHSR-KO and WT rats respectively ate $66.00 \%$ and $72.19 \%$ of their baseline daily food ration during this meal (this did not differ between the strains: $[\mathrm{t}(17)=-0.863$, $p=0.400])$. Rats only ate an average of 3 grams of chow during the last hour of the meal. Importantly, GHSR-KO rats ate less cookie dough than WT rats on the day of testing, despite having consumed a similar meal (absolute cookie dough: $[\mathrm{t}(17)=-2.048, \mathrm{p}=$ 0.056]; cookie dough per grams of body weight: $[\mathrm{t}(17)=-2.660, \mathrm{p}=0.016]$ ) (Figure 4). 

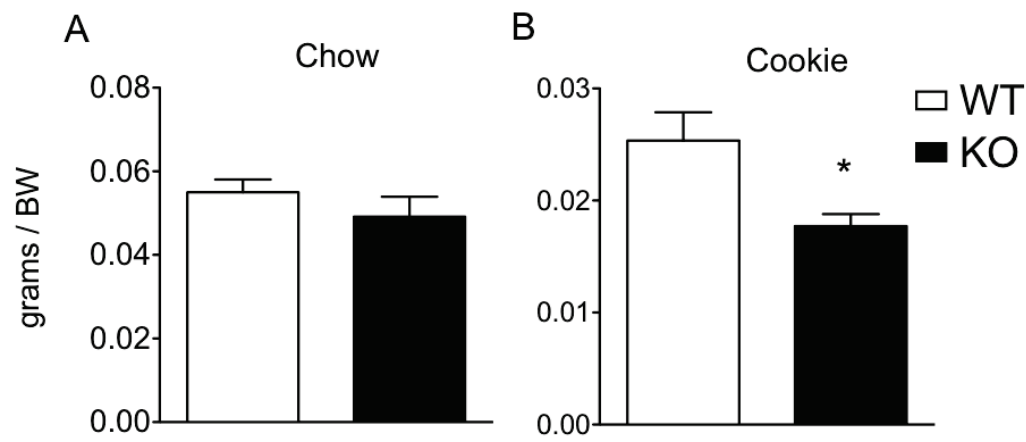

Figure 4. Grams of chow (A) and cookie dough (B) eaten by GHSR-KO and WT rats per grams of body weight during the last meal Experiment 1A. 


\section{Experiment 1B:}

Weight and food intake: GHSR-KO rats were significantly heavier than WT rats at all points during the experiment (Table 3; baseline (average of 3 days): $[\mathrm{t}(18)=2.249$, $\mathrm{p}=0.037]$; food restriction: $[\mathrm{F}(1,18)=5.889, \mathrm{p}=0.026])$, despite eating comparable amounts of food (baseline (average of 3 days): [t(18) $=0.400, p=0.694]$; food restriction: $[\mathrm{F}(1,18)=0.134, \mathrm{p}=0.719])$. Both strains were similar in terms of food intake relative to body weight (baseline: $[\mathrm{t}(18)=-1.586, \mathrm{p}=0.130]$; food restriction: $[\mathrm{F}(1,18)=1.7656, \mathrm{p}<0.001])$. All rats lost weight $[\mathrm{F}(22,396)=22.728, \mathrm{p}<0.001]$ and increased their food intake (absolute food intake: $[\mathrm{F}(22,396)=24.010, \mathrm{p}<0.001]$; food intake / body weight: $[\mathrm{F}(22,396)=25.230, \mathrm{p}<0.001])$ over 23 days of food restriction. There was no strain by day interaction effect in weight $[F(22,396)=0.530, p=0.962]$ or food intake (absolute food intake: $[\mathrm{F}(22,396)=0.863, \mathrm{p}=0.644]$; food intake $/$ body weight: $[F(22,396)=0.927, p=0.559])$ during this period. 
Table 3. Food intake (FI) and body weight (BW) from GHSR-KO and WT rats in Experiment 1B

\begin{tabular}{|c|c|c|c|c|}
\hline & & Food intake (g) & Body weight (g) & FI/BW \\
\hline \multirow{2}{*}{ Baseline (average) } & WT & $\begin{array}{c}24.42 \\
(+/-0.73)\end{array}$ & $\begin{array}{c}330.45 \\
(+/-7.50)^{*}\end{array}$ & $\begin{array}{c}0.075 \\
(+-0.009)\end{array}$ \\
\hline & $\mathrm{KO}$ & $\begin{array}{c}24.80 \\
(+/-0.59) \\
\end{array}$ & $\begin{array}{c}357.02 \\
(+/-9.12)^{*}\end{array}$ & $\begin{array}{c}0.070 \\
(+/-0.008) \\
\end{array}$ \\
\hline \multirow{2}{*}{$\begin{array}{l}\text { Food restriction: } \\
\text { day } 1\end{array}$} & WT & $\begin{array}{c}4.20 \\
(+/-0.72) \\
\end{array}$ & $\begin{array}{c}318.60 \\
(+/-9.14)^{*}\end{array}$ & $\begin{array}{c}0.013 \\
(+/-0.009) \\
\end{array}$ \\
\hline & $\mathrm{KO}$ & $\begin{array}{c}4.10 \\
(+/-0.72) \\
\end{array}$ & $\begin{array}{c}347.10 \\
(+/-9.14)^{*}\end{array}$ & $\begin{array}{c}0.012 \\
(+/-0.004) \\
\end{array}$ \\
\hline \multirow{2}{*}{$\begin{array}{l}\text { Food restriction: } \\
\text { day } 23\end{array}$} & WT & $\begin{array}{c}16.60 \\
(+/-1.23)^{\#} \\
\end{array}$ & $\begin{array}{c}305.60 \\
(+/-8.37)^{* \#}\end{array}$ & $\begin{array}{c}0.054 \\
(+/-0.011)^{\#}\end{array}$ \\
\hline & $\mathrm{KO}$ & $\begin{array}{c}15.20 \\
(+/-1.23)^{\#} \\
\end{array}$ & $\begin{array}{c}330.60 \\
(+/-8.37)^{* \#}\end{array}$ & $\begin{array}{c}0.045 \\
(+/-0.010)^{\#}\end{array}$ \\
\hline \multirow{2}{*}{ Testing day } & WT & $\begin{array}{c}16.80 \\
(+/-0.76) \\
\end{array}$ & $\begin{array}{c}306.90 \\
(+/-7.64) \\
\end{array}$ & $\begin{array}{c}0.055 \\
(+/-0.003) \\
\end{array}$ \\
\hline & $\mathrm{KO}$ & $\begin{array}{c}16.42 \\
(+/-0.96) \\
\end{array}$ & $\begin{array}{c}327.00 \\
(+/-7.07) \\
\end{array}$ & $\begin{array}{c}0.050 \\
(+/-0.003) \\
\end{array}$ \\
\hline
\end{tabular}

$*=$ significantly different from other strain on the same day $(\mathrm{p}<0.05)$

${ }^{*}=$ significantly different from day 1 of food restriction $(\mathrm{p}<0.05)$ 
ICH: A series of 2-way factorial ANOVAs with genotype and cookie dough exposure condition as between subject factors revealed several main effects of exposure condition on c-fos activation in the areas and cell populations processed for ICH (Figures 5 and 6). Relative to rats in other groups, rats that ate cookie dough had a greater percentage of $c$-fos activated orexin $B$ cells in the PFA $[F(2,32)=66.642, p<0.001]$ and LHA $[\mathrm{F}(2,32)=21.465, \mathrm{p}<0.001]$, as well as greater c-fos activation in other cell populations within these areas that did not express orexin $\mathrm{B}(\mathrm{PFA}:[\mathrm{F}(2,32)=7.340, \mathrm{p}=$ 0.002]; LHA: $[\mathrm{F}(2,32)=12.286, \mathrm{p}<0.001])$ or orexin A $(\operatorname{PFA}:[F(2,32)=0.1 .156, \mathrm{p}=$ $0.327, \mathrm{NS}]$; LHA: $[\mathrm{F}(2,32)=3.768, \mathrm{p}=0.034])$. In addition, rats that ate cookie dough had a greater percentage of c-fos activated TH-positive cells of the VTA $[F(2,32)=$ 26.213, $\mathrm{p}<0.001$ ], but reduced c-fos activation in VTA cells that did not express TH $[F(2,32)=3.884, p=0.034]$ than rats who did not (whether they had indirect exposure to it or not). Rats in this group also had greater c-fos activation in the arcuate nucleus $[F(2,32)=8.477, p=0.001]$ and the core $[F(2,32)=40.555, p<0.001]$ and shell $[F(2,32)$ $=15.175, \mathrm{p}<0.001]$ of the nucleus accumbens than rats in the other 2 groups.

Rats that were exposed to cookie dough cues but had no direct access to it had a greater percentage of c-fos activated orexin A cells in the PFA than rats who either ate cookie dough before sacrifice or had no exposure to it $[\mathrm{F}(2,32)=3.276, \mathrm{p}=0.051]$. Finally, compared to rats that had no exposure to cookie dough before sacrifice, rats in the other 2 groups had greater $\mathrm{c}-$ fos activation in the central $[(2,32)=6.452, \mathrm{p}=0.004]$ and basolateral $[\mathrm{F}(2,32)=3.386, \mathrm{p}=0.046]$ amygdala . 


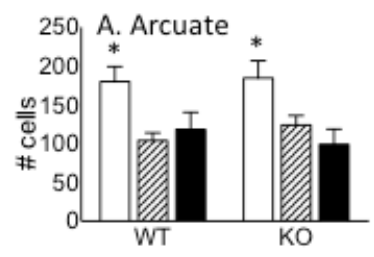

$\square$ Ingestion

西 Sight/Smell

- No Exposure
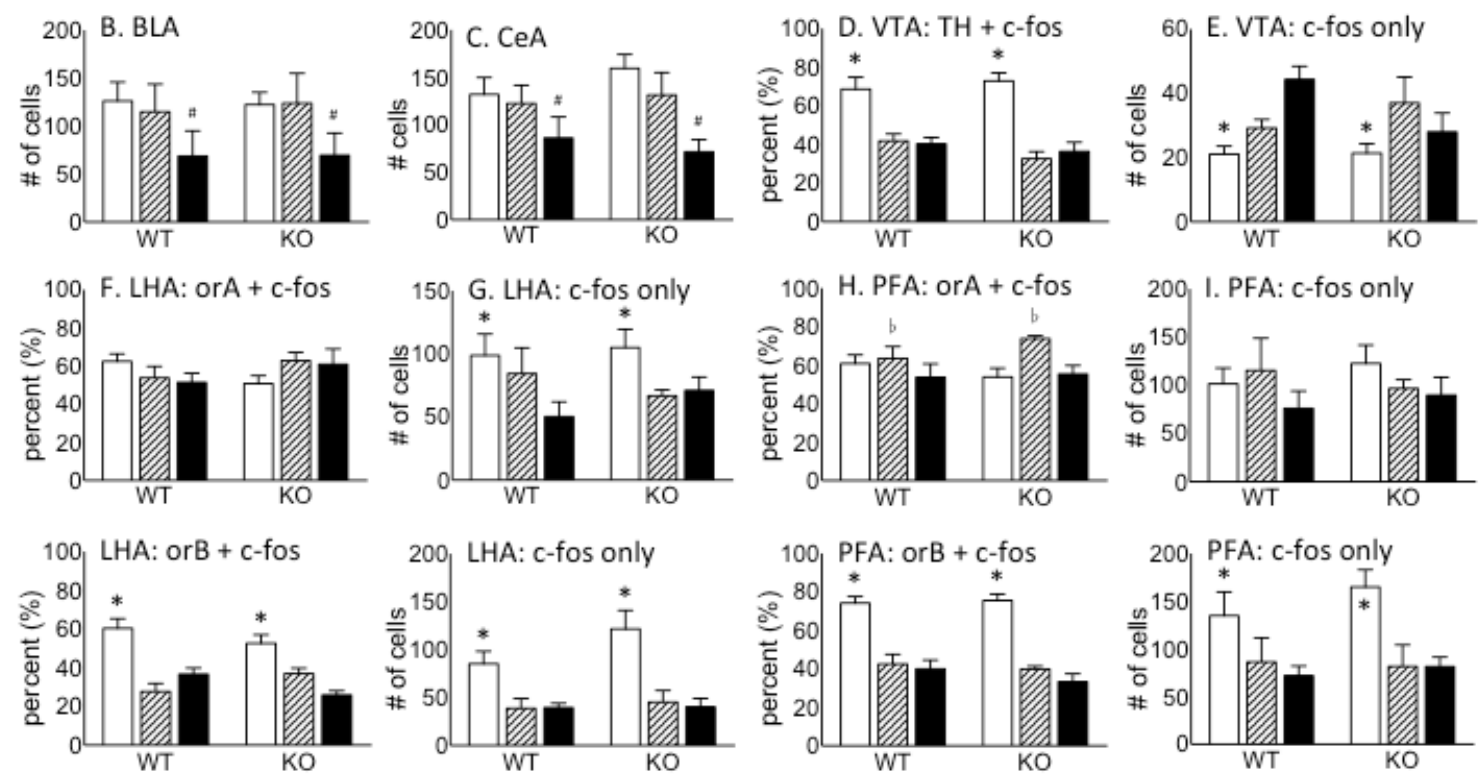

Figure 5. Number of c-fos positive cells in the arcuate (A), amygdala (B, C), VTA (E), LHA (G, K), PFA (I, M) and percentage of c-fos activated TH-positive cells (VTA: D), orexin A cells (LHA: F; PFA: H), and orexin B cells (LHA: J; PFA: L) in GHSR-KO and WT rats that ate cookie dough, saw/smelled cookie dough, or had no exposure to cookie dough in Experiments 1A and 1B. * = main effect of cookie dough exposure, with ingestion grp $\neq$ other groups $(p<0.05) ; b=$ main effect of cookie dough exposure, with sight/smell group $\neq$ other groups $(\mathrm{p}<0.05)$; $\#=$ main effect of cookie dough exposure, with the nothing grp $\neq$ other groups $(\mathrm{p}<0.05)$. 


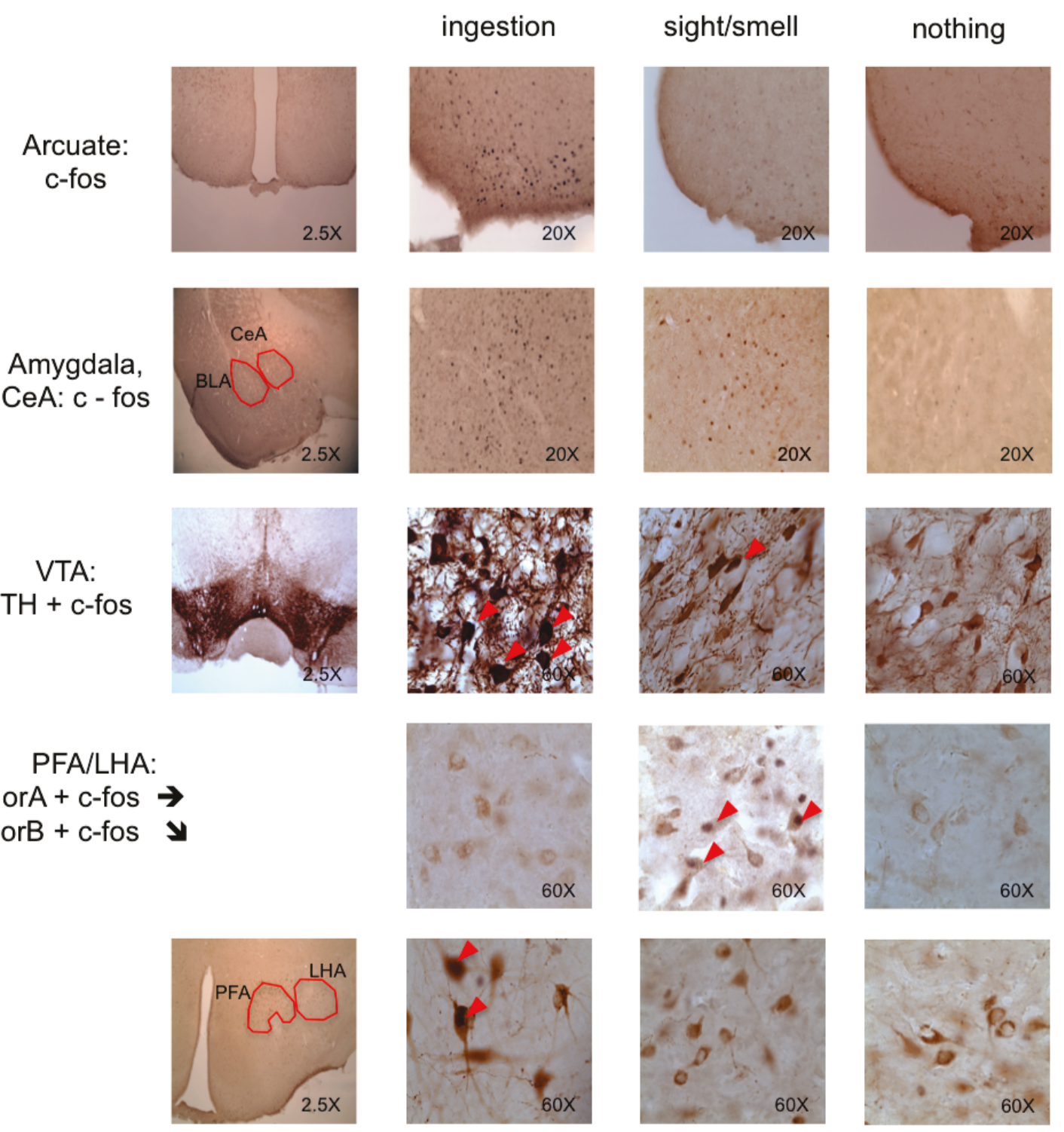

Figure 6. Representative stains in the arcuate nucleus (c-fos), amygdala (c-fos), VTA $(\mathrm{TH}+\mathrm{c}$-fos), LHA and PFA (orexin A $+\mathrm{c}-$ fos; orexin $\mathrm{B}+\mathrm{c}-$ fos) in GHSR-KO and WT rats that ate cookie dough, saw/smelled cookie dough, or had no exposure to cookie dough in Experiments 1A and 1B. Red arrows represent double-stained cells. 
All of the aforementioned c-fos effects were independent of genotype. In contrast, a significant interaction effect indicated that exposure to cookie dough cues was associated with greater c-fos activation in the NAC core of WT, but not GHSR-KO rats (2-way ANOVA: $[\mathrm{F}(2,32)=5.376, \mathrm{p}=0.010]$; independent t-test comparing WT and KO rats in that group: $[\mathrm{t}(7)=-6.270, \mathrm{p}<0.001])$ (Figure 7). 

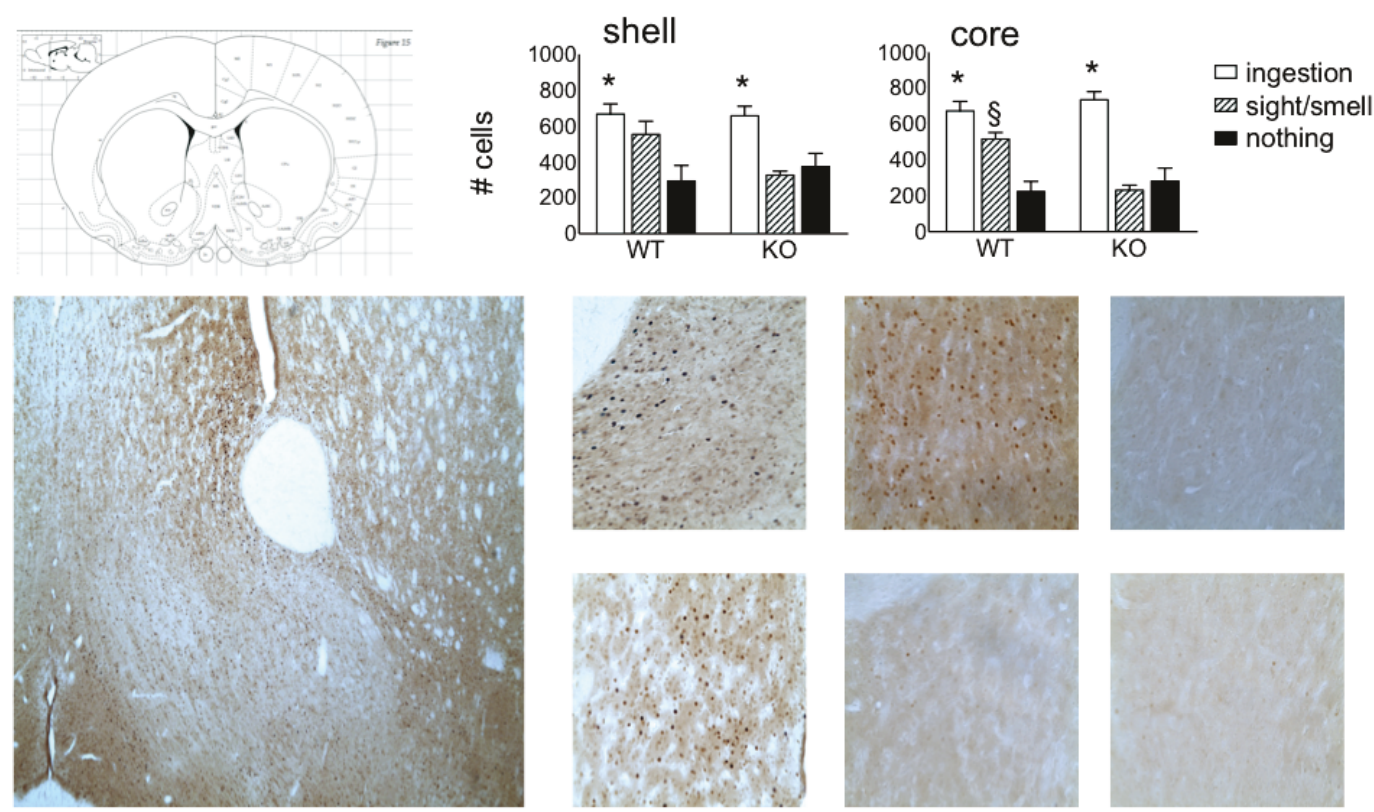

WT (core)

ingestion

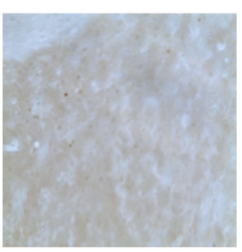

sight/smell

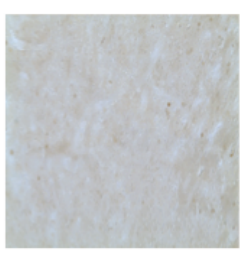

$\mathrm{KO}$ (core)

Figure 7. Number of c-fos activated cells in the nucleus accumbens shell and core in GHSR-KO and WT rats that ate cookie dough, saw/smelled cookie dough, or had no exposure to cookie dough in Experiments 1A and 1B (top) and representative stains (bottom). ${ }^{*}=$ main effect of cookie dough exposure, with ingestion grp $\neq$ other groups $(\mathrm{p}<0.05) ; \S=$ strain by exposure condition interaction $(\mathrm{p}<0.05)$. 
Finally, a series of Pearson correlations was performed to examine the relationship between c-fos activation in all areas and cell populations processed with $\mathrm{ICH}$ and the amount of cookie dough eaten at the end of a meal relative to body weight (rats in group 1). The analyses revealed that c-fos activation in the BLA was positively correlated with grams of cookie dough ingested by grams of body weight in WT, but not GHSR-KO rats (WT rats: $[r=0.706, p=0.022]$; KO rats: $[r=-0.106, p=0.787])$.

\section{Discussion}

Experiment $1 \mathrm{~A}$ used a paradigm designed to model dessert consumption in humans and found that GHSR-KO rats eat less of a tasty treat following a meal than their WT counterparts. Although the GHSR-KO rat strain is a relatively new model, the current data are consistent with previous reports of attenuated reward processing in these rats (Cahill, Hatchard, Abizaid, \& Holahan, 2014; Clifford et al., 2012; Wellman et al., 2012). Importantly, the fact that GHSR-KO rats still consumed a substantial amount of cookie dough after a regular chow meal indicates that although reward-related behaviours are attenuated in these rats, they are not absent. This is also consistent with previous studies using these rats (Clifford et al., 2012; Wellman et al., 2012). Beyond the GHSR$\mathrm{KO}$ rat model, our findings are consistent with a number of studies showing that mice genetically engineered to lack ghrelin or GHSR signalling are less responsive to both natural and artificial rewards (Abizaid et al., 2006; Bahi et al., 2013; Egecioglu et al., 2010; Jerlhag, Landgren, Egecioglu, Dickson, \& Engel, 2011; Jerlhag et al., 2009). This includes a study using a mouse KO for the enzyme GOAT (which octanoylates and activates ghrelin): upon exposure to a high fat diet in the last hour of a meal, GOAT-KO mice consumed less than WT mice (Davis et al., 2012). Our data also corroborate a 
growing body of literature showing that exogenous ghrelin enhances (and GHSR antagonists attenuate) the behavioural and physiological effects of rewards and that ghrelin can, on its own, act as a reinforcer (Perelló \& Zigman, 2012). Finally, the current findings support claims that in the presence of palatable foods, ghrelin signalling can make sated animals behave like hungry ones (King et al., 2011; Perello et al., 2010; Skibicka et al., 2013).

Meal anticipation helps animals prepare for the ingestion, absorption and metabolism of nutrients (Woods, 1991). In many species, meal anticipation is comprised of concurrent increases in locomotor activity and circulating ghrelin (Cummings et al., 2001; Drazen et al., 2006; Mistlberger, 1994; Sugino et al., 2002). In nocturnal rodents, shifting mealtime to a few hours during the light period causes shifts in food anticipatory activity (FAA) and ghrelin secretion (LeSauter et al., 2009; Stephan, 2002; Verbaeys et al., 2011). In the current study, such food restriction caused a drastic reduction in WT rats' dark phase activity. Conversely, their locomotor activity 1 hour before meal presentation increased such that it was significantly higher than that of KO rats, whereas no such strain differences were observed at that time during baseline. These findings are consistent with previous reports that ghrelin enhances (and GHSR antagonists reduce) FAA (LeSauter et al., 2009; Merkestein et al., 2012; Verhagen et al., 2011) and that FAA is attenuated in mice lacking the gene for either ghrelin or the GHSR [(Blum et al., 2009; Davis, Choi, Clegg, \& Benoit, 2011; LeSauter et al., 2009; Verhagen et al., 2011) - but see (Patton \& Mistlberger, 2013)]. As previously reported, FAA was not completely abolished in GHSR-KO rats (Blum et al., 2009; LeSauter et al., 2009; Verhagen et al., 2011). GHSR-KO rats' attenuated FAA did not translate into a compromised ability to 
adjust food intake when food restricted. Although some ghrelin/GHSR KO animals exhibit poorer rebound feeding in response to an acute fast (Abizaid et al., 2006), some have been shown to adapt to more prolonged food restriction regimens as efficiently as their WT counterparts (Blum et al., 2009; Davis et al., 2011).

In Experiment 1B, GHSR-KO rats were heavier than WT rats at baseline and during a food restriction regimen, despite eating comparable amounts of chow in both conditions. Strain differences in body weight might result from different locomotion patterns since in Experiment 1A, GHSR-KO rats moved less than WT rats, especially at night. These results were unexpected since other ghrelin and GHSR KO models are leaner than WT animals (Davis et al., 2011; Patterson et al., 2010; Zigman et al., 2005). It should be noted however that several ghrelin and GHSR KO models do not display clear feeding and body weight phenotypes (Sun et al., 2003; Wortley et al., 2004; Zhao et al., 2010). Similarly, studies examining the effects of ghrelin signalling on locomotion have yielded inconsistent results (Jászberényi, Bujdosó, Bagosi, \& Telegdy, 2006; Jerlhag et al., 2007; Nisembaum, de Pedro, Delgado, \& Isorna, 2014; Pfluger et al., 2011; TangChristensen et al., 2004) and ghrelin and GHSR KO models were reported to be more (Pfluger et al., 2008; Waddington Lamont, Bruton, Blum, \& Abizaid, 2014; Wortley et al., 2005), less (Zigman et al., 2005), and as active (Blum et al., 2009; Esposito, Pellinen, Kapás, \& Szentirmai, 2012; Verhagen et al., 2011) relative to WTs. Inconsistencies across genetic models may partly be explained by differences in dietary history, food availability and the genetic background of the animals (Sun, Butte, Garcia, \& Smith, 2008; Wortley et al., 2005). Nevertheless, the current data should be replicated as an increase in body weight was not reported in previous studies using GHSR-KO rats 
(Bülbül et al., 2011; Cahill et al., 2014; Clifford et al., 2011) and was also not observed in Experiment 1A (testing day).

Immunohistochemical analyses compared c-fos activation in brain areas relevant to food reward in GHSR-KO and WT rats that received direct exposure (with possibility of consumption), indirect exposure (no possibility of consumption), or no exposure to cookie dough at the end of their last meal. We found that ingesting cookie dough increased neuronal activation in the arcuate nucleus of the hypothalamus in both GHSR$\mathrm{KO}$ and WT rats. As mentioned in chapter 1, arcuate neurons respond to a number of peripheral hormones and metabolites that signal changes in energy balance and are responsible for their transduction into neural signals. These neurons activate orexigenic and anorexigenic pathways via their projections to other hypothalamic nuclei, including the lateral hypothalamus area (LHA). The latter can in turn modulate the activation of the mesolimbic reward system (reviewed in Schneeberger, Gomis, \& Claret, 2014).

Not surprisingly, cookie dough ingestion also led to increased neuronal activation in a number of the highly interconnected structures that form the mesolimbic reward system in both strains. These structures included the VTA (tyrosine hydroxylase (TH)positive neurons), nucleus accumbens (NAc), amygdala and the LHA (and adjacent perifornical area-PFA); natural and artificial rewards (including a high fat diet) have been shown to induce c-fos in all of these areas (Park \& Carr, 1998; Rorabaugh, Stratford, \& Zahniser, 2014; Valdivia, Patrone, Reynaldo, \& Perello, 2014).

TH-positive neurons in the VTA secrete the dopamine (DA) central to the functioning of the reward system. A large body of literature links the activity of these cells to reward processing (Wise, 2004). Primary reinforcers engage them, particularly 
when they are unexpected (as in the current study) (van Zessen et al 2012). Cookie dough ingestion also decreased the activity of non-TH VTA cells and increased activity in the NAc, a primary target of VTA DA cells. Given that $65 \%$ of VTA cells are dopaminergic, $30 \%$ GABAergic and only 5\% glutaminergic (van Zessen et al 2012), it is likely that the former effect reflects a reduced activation of GABAergic cells. These neurons exert a tonic inhibition on VTA DA cells, preventing DA release in the NAc (Jhou, Fields, Baxter, Saper, \& Holland, 2009; B. Johnson \& North, 1992; Westerink \& Kwint, 1996). Therefore, our findings are consistent with a scenario in which a palatable food reward both excites VTA DA cells and relieves them from tonic inhibition of local GABAergic cells, ultimately promoting the release of DA in the NAc and subsequent NAc activation.

Cookie dough ingestion increased c-fos activation in both the shell and the core of the NAc. The NAc is often described as the interface between the affective component of reward processing and the behavioural sequences required for reward seeking (i.e. turning motivation into action) (reviewed in Kelley, 2004). Eating cookie dough also increased c-fos activation in the basolateral (BLA) and central (CeA) parts of the amygdala, a structure well known for its role in associative learning (Gallagher \& Holland, 1994). As mentioned in chapter 1, the BLA receives sensory information about reinforcers and is important for the association with the neutral events that predict them. This information is sent to the CeA, which has a more direct role in guiding behaviour (reviewed in Everitt, Cardinal, Parkinson, \& Robbins, 2003).

Cookie dough ingestion increased c-fos activation in orexin B cells in the PFA and LHA. These areas have reciprocal connections with all of the structures mentioned above. Together with these structures, cells in the LHA and PFA regulate the response to 
rewards and drive food seeking (reviewed in Kenny, 2011). Whereas orexin A has affinity for both orexin receptors (OX1R and OX2R), orexin B only binds to the orexin receptor 2 (Sakurai et al., 1998). Some evidence suggests that orexin B (and OX2R signaling) plays an important role in arousal and the maintenance of wakeful states, and that orexin A (and OX1R signaling) is involved in reward processing and the response to environmental stimuli (Akanmu \& Honda, 2005; Baimel et al., 2014; Plaza-Zabala, Maldonado, \& Berrendero, 2012; Sakurai \& Mieda, 2011; Smith, See, \& Aston-Jones, 2009; Wang, You, \& Wise, 2009). Nevertheless, VTA DA cells express both orexin receptors (Narita et al., 2006) and both orexin isoforms can increase their firing rate (Korotkova, Sergeeva, Eriksson, Haas, \& Brown, 2003). Further, OX2R antagonists can decrease the behavioural response to some reinforcers (Brown, Khoo, \& Lawrence, 2013; Di Sebastiano, Wilson-Pérez, Lehman, \& Coolen, 2011). Under the current experimental conditions, eating cookie dough may have activated both a reward processing network and one that promotes alertness.

Eating cookie dough did not induce c-fos in orexin A cells. Although some studies show orexin A cell activation in response to rewards (Valdivia et al., 2014), Harris and colleagues (Harris, Wimmer, Randall-Thompson, \& Aston-Jones, 2007) report that similar effects only occur when the reward is presented in a novel environment. Finally, cookie dough ingestion increased c-fos in LHA and PFA cells that did not express any orexin. This might reflect an increase in the activation of other cell types, such as melanin-concentrating hormone containing neurons, or GABAergic leptinresponsive neurons (reviewed in Fulton, 2010). 
Rats that were allowed to see and smell, but not eat, cookie dough showed an increase in c-fos activation in the amygdala that was similar to that seen after cookie dough ingestion. The amygdala is highly sensitive to emotionally relevant stimuli (Baxter $\&$ Murray, 2002). In humans, it contains neurons that respond to the sight of food (Arana et al., 2003; Killgore et al., 2003). In rats, the BLA and CeA mediate aspects of cuepotentiated feeding and the conditioned response to reward cues (Holland \& Gallagher, 2003; Petrovich \& Gallagher, 2003; Petrovich, Holland, \& Gallagher, 2005). Indirect cookie dough exposure also induced c-fos activation in orexin A cells in the PFA. This is consistent with previous reports linking reward cues/expectation with the activity of these cells (Choi et al., 2010; Harris et al., 2005). Although it was previously suggested that orexin A cells in the LHA and PFA mediate the preference for reward-related cues and arousal respectively (Harris \& Aston-Jones, 2006), the current results do not support this view. Similarly, Choi and colleagues (2010) found that orexin A cells in the PFA, but not the LHA, are activated in anticipation of a chocolate treat. PFA-orexin is also important for sex and cocaine reinforcement (Di Sebastiano et al., 2011; Hamlin, Clemens, \& McNally, 2008).

All of the c-fos effects mentioned so far were consistent across GHSR-KO and WT rats. Interestingly, indirect exposure to cookie dough increased c-fos activation in the NAc core of WT but not GHSR-KO rats. As mentioned in chapter 1, the NAc core and shell differ in their connections and functions. The shell is intimately connected with a number of limbic structures (ex: VTA, LHA, hippocampus, and amygdala) and is important for the rewarding properties of drugs and foods, as well as the motivation to obtain them (Kelley, 2004; McFarland \& Kalivas, 2001). The core is more closely related 
to motor structures (ex: substantia nigra, subthalamic nucleus, dorsolateral ventral pallidum) and has overlapping functions with the rest of the striatum (reviewed in Kelley, 2004). It is involved with the execution of instrumental action, and importantly, the behavioural response to cues that signal rewards (Floresco, McLaughlin, \& Haluk, 2008; Fuchs et al., 2004; Kelley, 2004). The fact that there was a strain difference in the core, but not the shell of the NAc, may indicate that GHSR-KO and WT rats have similar emotional response to palatable food cues, but that a stronger neuronal response in the NAc core in WT rats is what pushes them to overeat a treat when it is readily available. Further augmentation of the core's involvement post-ingestion may have masked such strain differences in rats that were allowed to eat the cookie dough. Although this hypothesis remains to be tested directly, the idea that ghrelin is primarily involved in behavioural activation in response to reward cues is in line with a number of observations. For instance, ghrelin administration enhances (and GHRS antagonism decreases) cue-potentiated feeding (Kanoski, Fortin, Ricks, \& Grill, 2013; Walker, Ibia, \& Zigman, 2012). Endogenous ghrelin levels are correlated with reinstatement of cocaine seeking that is induced by cocaine cues (Tessari et al., 2007). In humans, pictures of palatable foods increase endogenous ghrelin levels (Schüssler et al., 2012), and ghrelin administration increases the fMRI response to such pictures (Malik, McGlone, Bedrossian, \& Dagher, 2008).

Given that ghrelin's effects on feeding and some reward behaviour require an intact orexin system (Cone, McCutcheon, \& Roitman, 2014; Miura et al., 2007; Perello et al., 2010; Toshinai et al., 2003), we initially predicted that strain differences in cookie dough ingestion would be paralleled by corresponding strain differences in orexin cell 
activation; this was not the case. It is possible that GHSR KO rats' reduced propensity towards dessert consumption is independent of the orexin system. An alternative, and perhaps more plausible explanation is that differential responses to orexin in areas downstream from the PFA and LHA (due to, for example, different orexin receptor numbers) underlie strain differences in cookie dough consumption. Consistent with this hypothesis is the fact that GOAT-KO mice have the same number of orexin cells and receptors in the hypothalamus as their WT counterparts, but fewer orexin receptors in the nucleus accumbens (Davis et al., 2012).

\section{Conclusion}

In conclusion, the current study used a novel rat GHSR-KO model to examine the effects of GHSR signalling on food anticipation and reward-based overeating, as well as the neuronal response to eating versus smelling/seeing a treat. The results support claims that ghrelin facilitates, but is not absolutely necessary for food anticipatory activity. Importantly, the data support claims that ghrelin facilitates food reward behaviours, and extends these findings by demonstrating that it is also involved in dessert consumption in sated rats. Again, absence of ghrelin signalling did not completely abolish cookie dough intake, suggesting that ghrelin is not the sole mediator of this behaviour. Finally, a preliminary attempt to shed light on the neural bases of ghrelin-facilitated dessert behaviour suggested that neurons in the NAc core might be involved; GHSR-KO rats had altered neuronal response to food cues (sight and smell) in this area, but not in the NAc shell, VTA, LHA/PFA, arcuate, or amygdala. Further, the lack of ghrelin signalling did not alter the neuronal response to cookie dough ingestion/anticipation in any of these areas. Future studies should further explore the neural bases of dessert consumption; 
particular focus should be given to elucidating ghrelin's effects on orexin receptor binding in the VTA and NAc. 


\section{Chapter 4: The role of ghrelin in reinstatement of food seeking - operant conditioning}

Hypothesis 2: ghrelin facilitates the reinstatement of palatable food seeking that is triggered by food priming and food associated cues.

Aim 3: to compare GHSR-KO and WT rats in the operant conditioning version of the reinstatement test.

\section{Introduction}

Relapse to preferred foods and unhealthy eating habits is a primary concern for people undergoing weight reduction diets (Elfhag \& Rössner, 2005; Kramer et al., 1989). As reviewed in chapter 1, such relapse is most frequently studied using an operant conditioning model that is also used in drug addiction research. Over the last decade, the use of this model has facilitated the identification of a number of peptides and neurotransmitters that act centrally to modulate the reinstatement of food seeking, many of which also impact reinstatement of drug seeking (Nair, Adams-Deutsch, Epstein, et al., 2009). These include, but are not limited to, orexigenic peptides such as orexin and melanin-concentrating hormone (Boutrel et al., 2005; Cippitelli et al., 2010; James et al., 2011; Nair, Adams-Deutsch, Epstein, et al., 2009; Nair et al., 2008; Richards et al., 2008; Wang et al., 2009). In this context, the role of ghrelin, the only circulating orexigenic hormone currently known, is relatively under-studied.

A role for ghrelin in the reinstatement of food seeking is suggested by the fact that ghrelin activates the HPA stress response system, and that stress is intimately linked with 
food seeking relapse in dieting individuals and rats tested in the reinstatement model (Asakawa et al., 2001; Ghitza et al., 2006; Greeno \& Wing, 1994; Grilo et al., 1989; Tassone et al., 2003). In addition, caloric restriction, which increases plasma ghrelin levels, facilitates reinstatement of both food and drug seeking (Nair, Adams-Deutsch, Epstein, et al., 2009). Elevated ghrelin levels are also associated with alcohol cravings in people who try to abstain from drinking, and such cravings often precede relapse episodes (Koob \& Volkow, 2010; Koopmann et al., 2012). Finally, endogenous ghrelin levels are positively correlated to the reinstatement of cocaine seeking in rats (Tessari et al., 2007).

In the current study, we sought to determine if ghrelin signalling was involved in the relapse to food reward seeking following priming (i.e. re-exposure to the food reward) and exposure to a cue previously associated with the reward by comparing GHSR-KO and WT rats in the reinstatement model. We chose sucrose and high fat chocolate flavoured pellets as the rewards because preferred foods are often sweet and rich in fat and ghrelin preferentially increases intake of sweet and high-fat foods (Disse et al., 2010; Shimbara et al., 2004).

\section{Methods: Experiment 2 (A \& B)}

\section{Subjects:}

Twenty-four rats (12 GHSR-KO and $12 \mathrm{WT}$ ) were used in experiment $2 \mathrm{~A}$ and 20 (10 GHSR-KO and $10 \mathrm{WT}$ ) were used in experiment 2B. Baseline food intake was measured for 3 days before the start of the experiments. The rats were subsequently food restricted as to maintain $85 \%$ of their baseline body weight. Testing began 5 days after 
the start of food restriction. All testing took place in the dark phase (reverse 12 hours dark/light cycle, lights off at 8:00AM).

Experiment 2A: Operant conditioning using a sweet, high fat reward

Operant conditioning: All animals were subjected to a single 6-hour autoshaping session during which high-fat chocolate-flavoured pellets were delivered every 5 minutes. Each pellet delivery was accompanied by a 2 second light/tone stimulus. Starting on the next day, rats were trained for 6 hours $(2 \times 3$ hours: from 9:00AM to 12:00PM and from 1:00PM to 4:00PM) every second day, for 22 days (11 training sessions). At the start of the first 5 sessions, 3 pellets were placed on the active lever in order to facilitate acquisition. During training, pressing on the active lever resulted in the delivery of a pellet and exposure to the food cue (light/tone stimulus). Each pellet delivery was followed by a 20 second timeout period, during which pressing on the lever had no consequence. Similarly, pressing on the inactive lever at any point had no programmed consequence. On training days, rats were fed their maintenance food ration 1 hour after the conditioning session. On "off" days, rats were fed 1 hour after the start of the dark phase.

One day after the completion of the training phase, rats were exposed to a series of 3-hour extinction sessions (starting at 9:00AM or 1:00PM, on alternating days), during which lever pressing (active or inactive) either had no programmed consequence. Rats received daily extinction sessions until they reached the extinction criterion, defined as pressing on the active lever less than 10 times over 3 hours on 3 consecutive days, with a minimum of 6 extinction sessions. 
Food seeking (i.e. active lever pressing) was reinstated by either pre-exposure to the food pellets (food priming) or contingent presentations of the light-tone food cue upon lever pressing. Reinstatement testing began 24 hours after rats reached the extinction criterion. Each rat was tested for both kinds of reinstatement (food primingand cue-induced) in four 3-hour sessions over the course of 5 days. Specifically, each rat was subjected to 2 sets of 2 sessions separated by 1 regular extinction session, with half of the rats receiving food priming-induced reinstatement session first and the other half receiving cue-induced reinstatement first. In the food priming reinstatement condition, 5 pellets were delivered in a non-contingent way at the start of the session. In the cue reinstatement session, rats were presented with the light/tone stimulus previously associated with pellet delivery 3 times at the start of the session, and upon each active lever press during the session. With the exception of tone/light stimulus presentations in the cue-induced reinstatement conditions, the reinstatement sessions themselves were identical to extinction ones.

\section{Experiment 2B: Operant conditioning using a sucrose reward}

Operant conditioning: Rats were trained to lever press to obtain sucrose pellets. Due to previous reports that 3,6 , and 9 hours of daily conditioning produce similar results in a paradigm similar to that used in the current study (Nair, Adams-Deutsch, Pickens, et al., 2009), training sessions were reduced to 3 hours per day on 12 consecutive days (autoshaping session was also 3 hours). Training sessions were conducted in the morning (9:00AM-12:00PM) or the afternoon (1:00PM-4:00PM), and this alternated each day. Apart from these modifications, training, extinction, and reinstatement proceeded as described for Experiment 2A. 
Brain monoamine analyses: Rats trained with sucrose pellets were left undisturbed and under ad lib feeding conditions for 2 to 3 weeks following reinstatement testing. On the last experimental day, half of the rats ( $5 \mathrm{KO}$ and $5 \mathrm{WT})$ were placed in the operant conditioning box for 5 minutes with only the house light on (i.e. lever pressing did not have any programmed consequences) and euthanized by rapid decapitation. The other rats were also euthanized by rapid decapitation but were not previously exposed to the operant conditioning box.

Tissue punches from the basolateral amygdala, prefrontal cortex, arcuate nucleus and accumbens nucleus were flash frozen and stored in homogenizing solution $(14.17 \mathrm{~g}$ monochloroacetic acid, 0.0168g EDTA, $5.0 \mathrm{ml}$ methanol, $500 \mathrm{ml}$ HPLC-grade water). Levels of serotonin (5HT), dopamine (DA), noradrenalin (NA), and their metabolites (5HT metabolite $=5$-hydroxyindolacetic acid (5-HIAA); DA metabolites $=3,4-$ dihydroxy-phenylacetic acid (DOPAC), 3-methoxytyramine (3MT), and homovanillic acid (HVA); NA metabolite = 3-methoxy-4-hydroxyphenylglycol (MHPG)) were assessed in each brain region using high-performance liquid chromatography (HPLC) as described in Anisman and Zacharko (Anisman \& Zacharko, 1990).

\section{Results: Experiment 2 (A \& B)}

\section{Experiment 2A:}

Operant conditioning: The number of pellets earned and the number of active lever time out responses increased significantly over the training phase (main effect of day: pellets earned: $[\mathrm{F}(10,220)=32.563, \mathrm{p}<0.001]$; time out responses: $[\mathrm{F}(10,220)=$ $16.887,<0.001])$, but remained similar across genotypes $(\mathrm{p}>0.05$ for both main effects of strain and strain $\mathrm{x}$ day interactions) (figure 8A). The number of reinforced and non- 
reinforced (i.e. timeout) active lever presses also remained similar throughout the training phase (day 1: $[\mathrm{t}(23)=0.399, \mathrm{p}=0.694]$; day 11: $\mathrm{t}(23)=-1.223, \mathrm{p}=0.234])$. The number of inactive lever presses did not increase over time and did not differ between GHSR-KO and WT rats (data not shown). During the extinction phase, there was a significant decrease in active lever presses $[\mathrm{F}(5,110)=53.743, \mathrm{p}<0.001]$, and no strain differences or strain by day interaction ( $\mathrm{p}>0.05$ in both cases) (figure $8 \mathrm{~B})$. GHSR-KO and WT rats took an average of 5.92 and 5.42 days respectively to reach the extinction criterion $(\mathrm{p}>$ 0.05) (figure $8 \mathrm{C}$ ).

A repeated measure ANOVA for active lever pressing with the reinstatement stimulus as the within factor and strain as the between factor was used to assess reinstatement of food seeking. The three levels of the reinstatement stimulus factor were: cue-induced reinstatement (average of 2 sessions), food priming-induced reinstatement (average of 2 sessions) and extinction. The analysis revealed a significant main effect of reinstatement stimulus $[\mathrm{F}(2,44)=9.904, \mathrm{p}<0.001]$, but no main effect of strain $[\mathrm{F}(1,22)$ $=0.036, \mathrm{p}=0.851]$ and no interaction of the 2 factors $[\mathrm{F}(2,44)=0.647, \mathrm{p}=0.528]$. A series of paired t-tests revealed that rats pressed on the active lever significantly more times during cue-induced reinstatement than during food priming-induced reinstatement $[\mathrm{t}(23)=5.820, \mathrm{p}=0.010]$ and an extinction session $[\mathrm{t}(23)=3.977, \mathrm{p}=0.001]$. There were no differences between food priming-induced reinstatement and an extinction session $[\mathrm{t}(23)=1.314, \mathrm{p}=0.202]$ (figure $8 \mathrm{D}$ ). 
Training

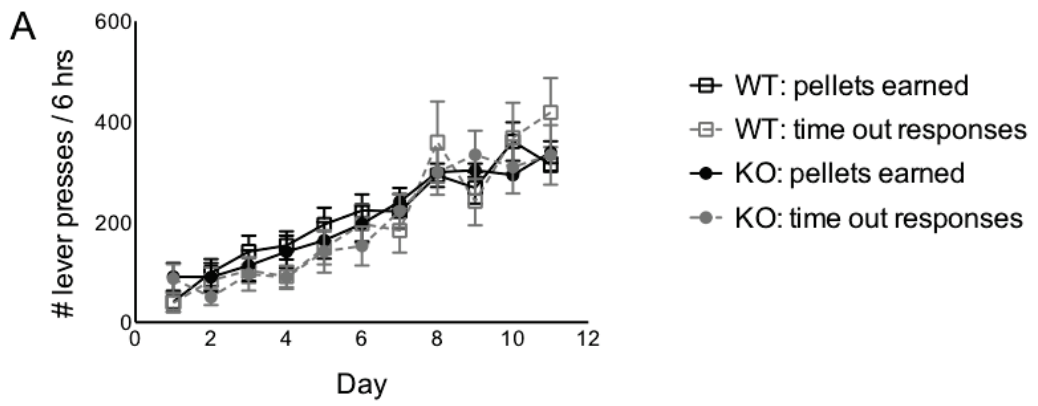

Extinction

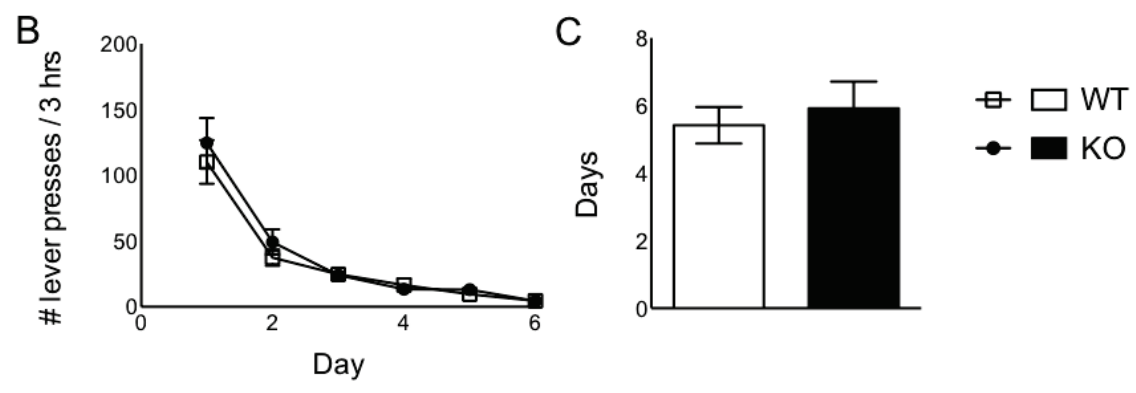

\section{Reinstatement}

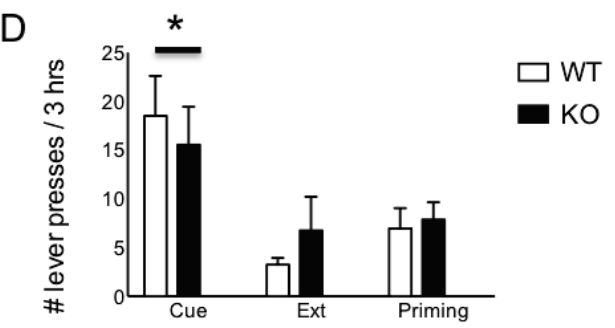

Figure 8. Operant conditioning in GHSR-KO and WT rats using high fat chocolateflavoured pellets as a reinforcer (Exp. 2A). A. Training: Reinforced (pellets earned) and non-reinforced (timeout responses) active lever presses. B. Extinction: Active lever presses. C. Number of days required to reach extinction criterion. D. Active lever pressing during cue- food priming-induced reinstatement and an extinction session (during the reinstatement phase). $*=$ main effect of reinstatement condition $(p<0.05)$. 
Experiment 2B:

Operant conditioning: A total of 4 rats $(3 \mathrm{KO}+1 \mathrm{WT})$ did not acquire the lever pressing response and were excluded from the analyses. Training for sucrose responding in Experiment 2B yielded similar results as training in Experiment 2A. The number of pellets earned and the number of active lever timeout responses increased significantly over time (main effect of day: pellets earned: $[\mathrm{F}(11,154)=36.665, \mathrm{p}<0.001]$; time out responses: $[\mathrm{F}(11,154)=14.493, \mathrm{p}<0.001])$, but remained similar across genotypes $(\mathrm{p}>$ 0.05 for both main effects of strain and strain $x$ day interactions) (figure 9A). The number of reinforced and non-reinforced active lever presses remained similar during the training phase (day 1: $[\mathrm{t}(15)=1.270, \mathrm{p}=0.223]$; day $12:[\mathrm{t}(15)=-1.824, \mathrm{p}=0.088])$. The number of inactive lever presses did not increase over time and did not differ between the strains (data not shown). During the extinction phase, there was a significant decrease in active lever presses over time $[F(5,70)=9.829, p<0.001]$, and no strain differences or strain by day interaction ( $p>0.05$ in both cases) (figure 9B). GHSR-KO rats took significantly longer to reach the extinction criterion than WT rats $[\mathrm{t}(14)=3.995, \mathrm{p}=0.001]$. The average numbers of days required by GHSR-KO and WT rats to reach the criterion were 15.86 and 10.56, respectively (figure 9C).

As in Experiment 2A, a repeated measure ANOVA for active lever pressing with reinstatement stimulus as the within factor and strain as the between factor was used to assess reinstatement. The three levels of the reinstatement stimulus factor were also the same as in Experiment 2A. Again, the analysis revealed a significant main effect of reinstatement stimulus $[\mathrm{F}(2,28)=5.362, \mathrm{p}=0.011]$, but no main effect of strain $[\mathrm{F}(1,14)$ $=1.693, \mathrm{p}=0.214]$ and no interaction of the 2 factors $[\mathrm{F}(2,28)=0.158, \mathrm{p}=0.855]$. A 
series of paired t-tests revealed that in this case, rats pressed on the active lever significantly more during the cue-induced reinstatement and the food priming-induced reinstatement sessions than during the extinction session (cue vs extinction: $[\mathrm{t}(15)=$ $2.409, \mathrm{p}=0.029]$; food priming vs extinction: $[\mathrm{t}(15)=4.809, \mathrm{p}<0.001])$. There was no difference between cue-induced reinstatement and food priming-induced reinstatement $[\mathrm{t}(15)=-0.390, \mathrm{p}=0.702]$ (figure 9D). 
Training

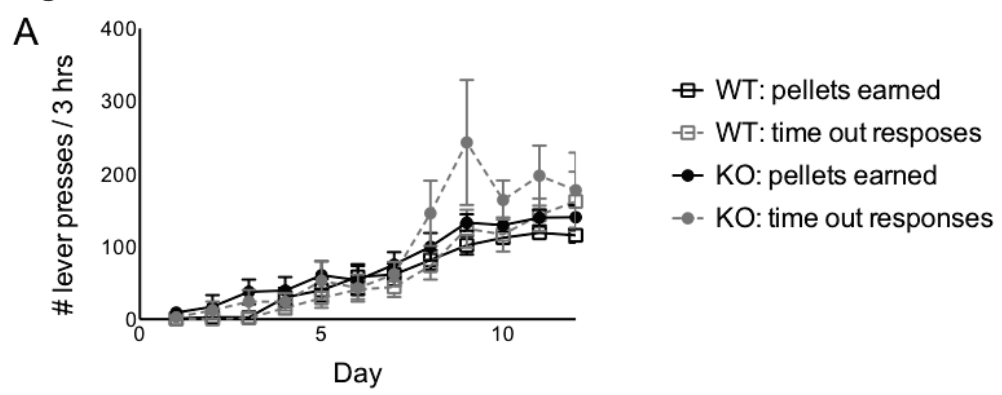

\section{Extinction}
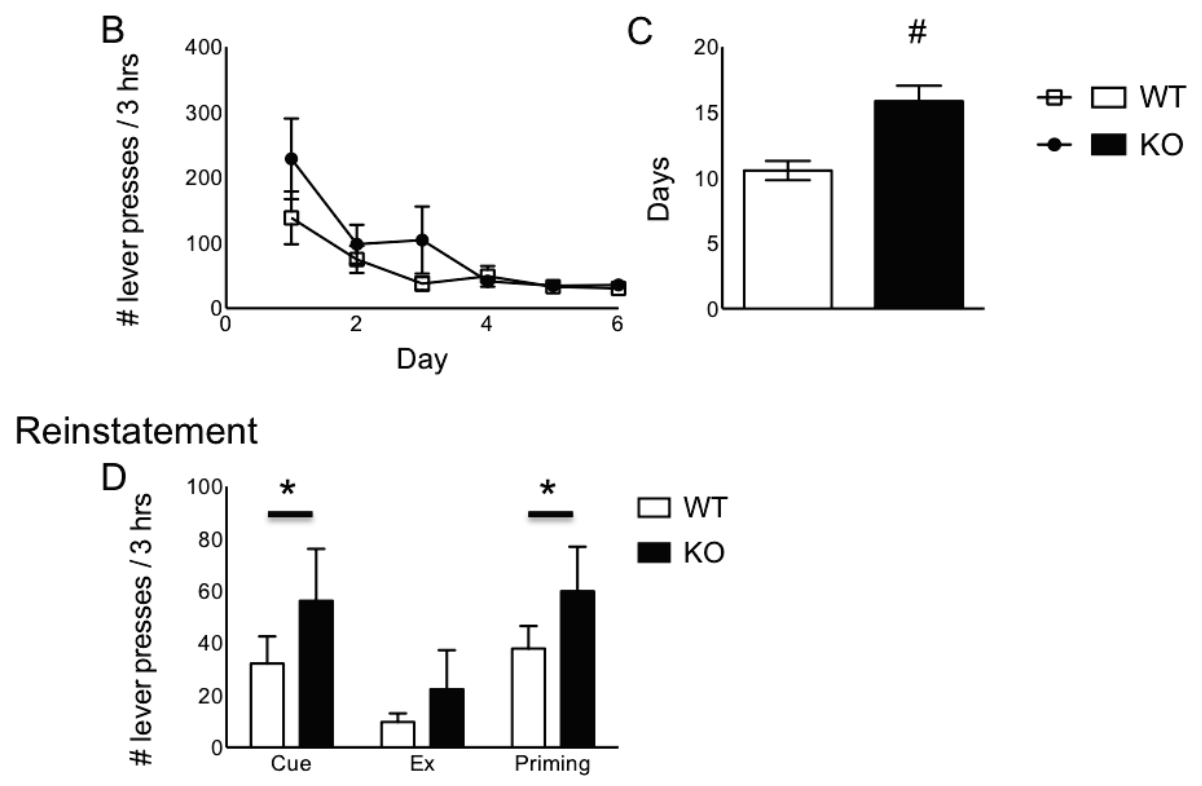

Figure 9. Operant conditioning in GHSR-KO and WT rats using sucrose pellets as a reinforcer (Experiment 2B). A. Reinforced (pellets earned) and non-reinforced (time out responses) active lever presses during training. B. Active lever presses during extinction. C. Number of days required to reach an extinction criterion. D. Active lever pressing during cue-induced reinstatement, food priming-induced reinstatement and an extinction session (during the reinstatement phase). $\#=$ strain difference $(\mathrm{p}<$ 0.05). ${ }^{*}=$ main effect of reinstatement condition $(\mathrm{p}<0.05)$. 
Brain monoamine analyses: Brain monoamine levels (as well as the ratios of monoamine neurotransmitters to their metabolites) were examined using a series of 2way ANOVAs with strain and operant box exposure (whether the rats were exposed to operant box before decapitation) as between subjects factors (Figures 10-13). Independent t-tests comparing exposure levels in GHSR-KO and WT rats separately were used as follow up analyses for significant main effects. The analyses revealed a number of significant strain differences. Relative to WT rats, GHSR-KO rats had reduced levels of prefrontal cortex HVA $(F 1,16)=12.066, p=0.003], 5 \mathrm{HT}[\mathrm{F}(1,16)=7.192, \mathrm{p}=0.016]$ and $\mathrm{NE}[\mathrm{F}(1,16)=10.570, \mathrm{p}=0.005]$. They also had lower $3 \mathrm{MT} / \mathrm{DA}$ ratios in the arcuate nucleus $[\mathrm{F}(1,14)=4.674, \mathrm{p}=0.048]$ and HVA/DA ratios in the nucleus accumbens $[F(1,16)=1.177, p=0.294]$. The analyses also revealed 2 significant main effects of operant box exposure: operant box exposure decreased 5HIAA levels $[F(1,14)=11.330$, $\mathrm{p}=0.005]$ and increased MHPG levels $[\mathrm{F}(1,14)=5.360, \mathrm{p}=0.036]$ in the amygdala of GHSR-KO and WT rats. Finally, operant box exposure decreased amygdala HVA and 5HT levels in GHSR-KO rats, but not WT rats (HVA interaction: $[\mathrm{F}(1,14)=10.273, \mathrm{p}=$ 0.006], KO rats: $[\mathrm{t}(6)=-3.355, \mathrm{p}=0.015]$, WT rats: $[\mathrm{t}(8)=0.601, \mathrm{p}=0.565] ; 5 \mathrm{HT}$ interaction: $[\mathrm{F}(1,14)=6.922, \mathrm{p}=0.020], \mathrm{KO}$ rats: $[\mathrm{t}(6)=-2.719, \mathrm{p}=0.035]$, WT rats: $[\mathrm{t}(8)=0.810, \mathrm{p}=0.441])$. 

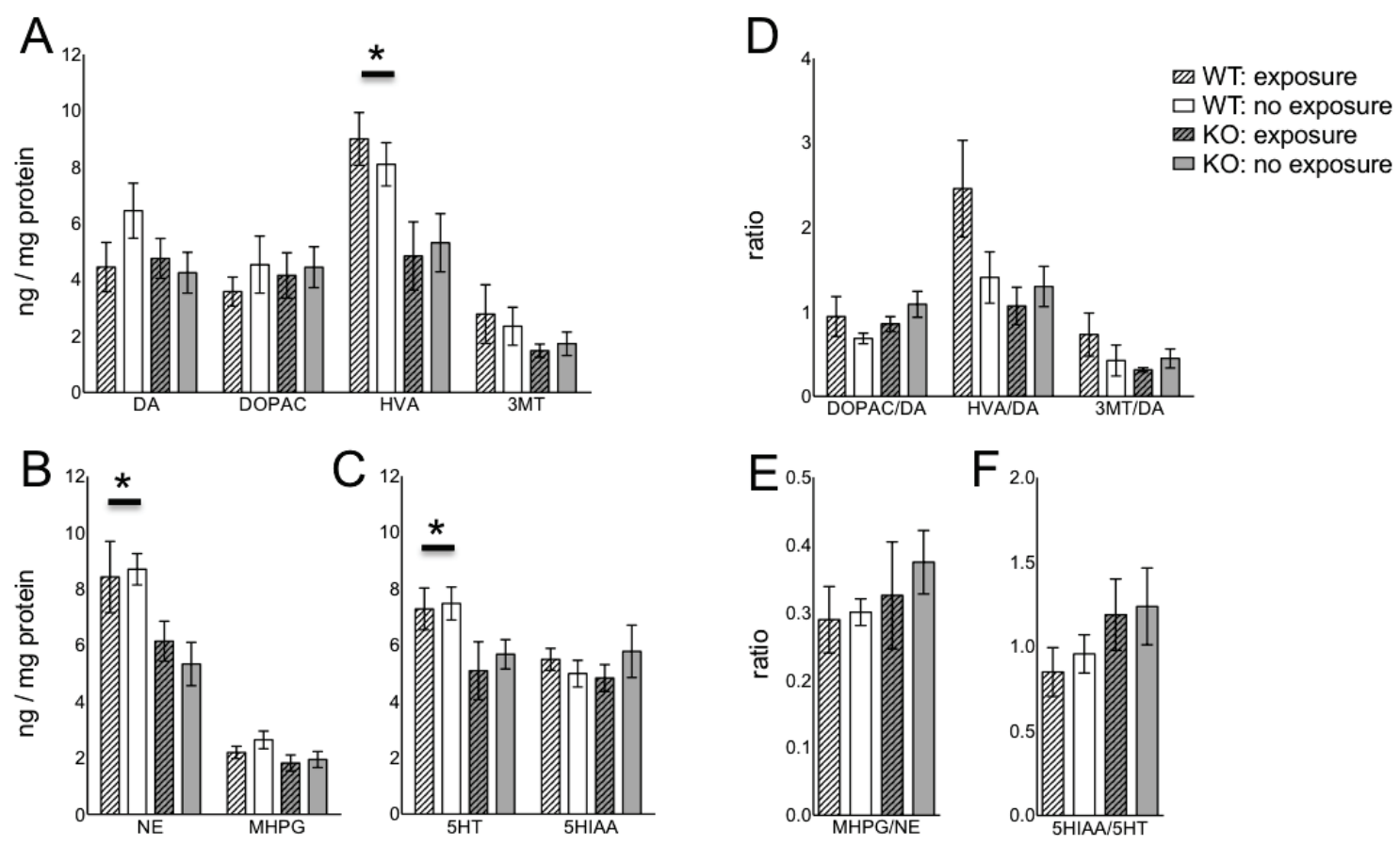

Figure 10. Mean levels (+/- SEM) of DA, NE, 5HT and their metabolites (A-C) and ratio of each monoamine to its metabolites (D-F) in the prefrontal cortex of GHSR-KO and WT rats in Experiment 2B. * denotes main effects of strain $(\mathrm{p}<0.05)$. 

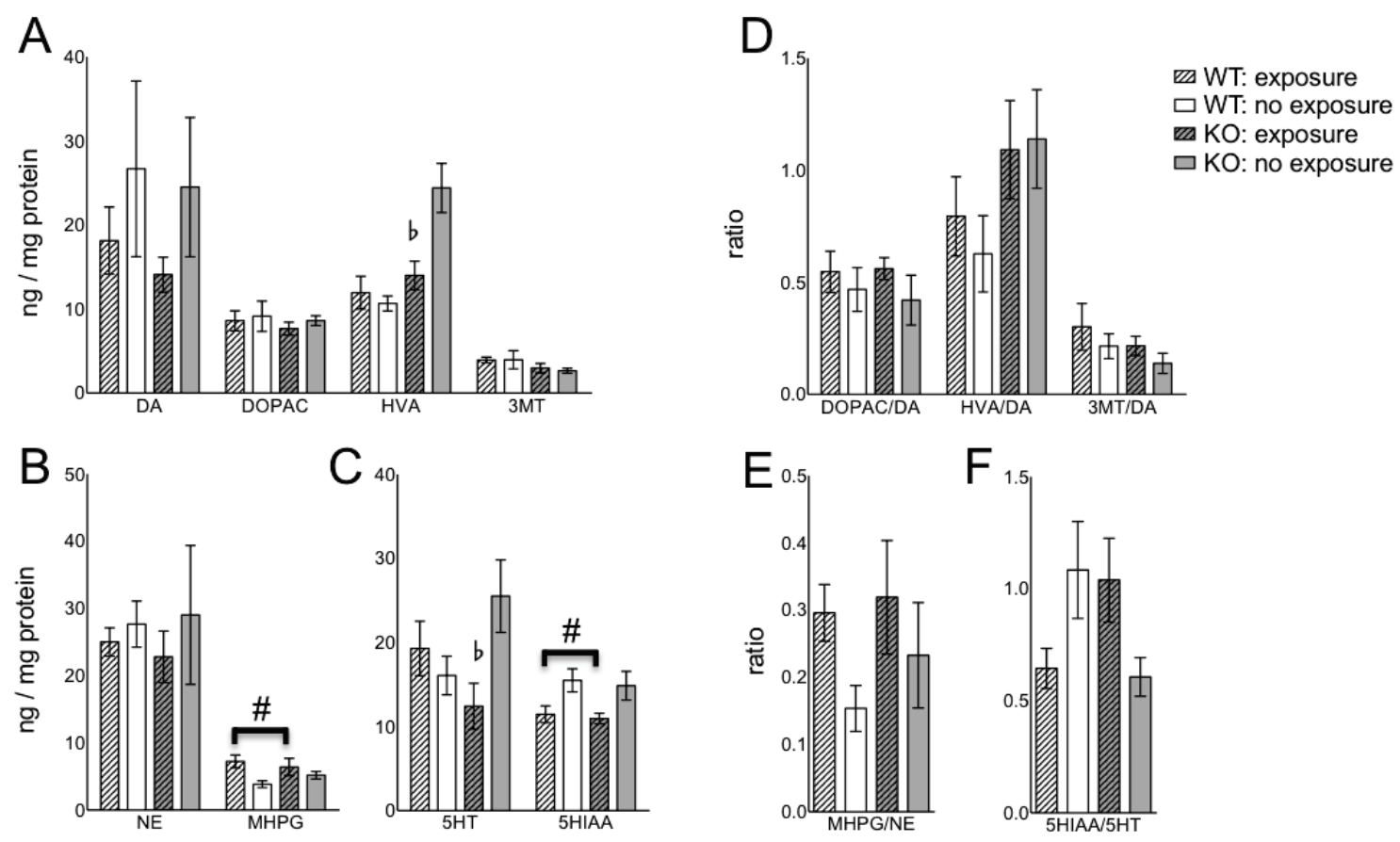

Figure 11. Mean levels (+/- SEM) of DA, NE, 5HT and their metabolites (A-C) and ratio of each monoamine to its metabolites (D-F) in the amygdala in GHSR-KO and WT rats in Experiment 2B. * denotes a significant strain effect $(\mathrm{p}<0.05)$. \# denotes a significant effect of operant box exposure $(\mathrm{p}<0.05)$. bdenotes a significant operant box exposure by strain effect $(p<0.05)$. 

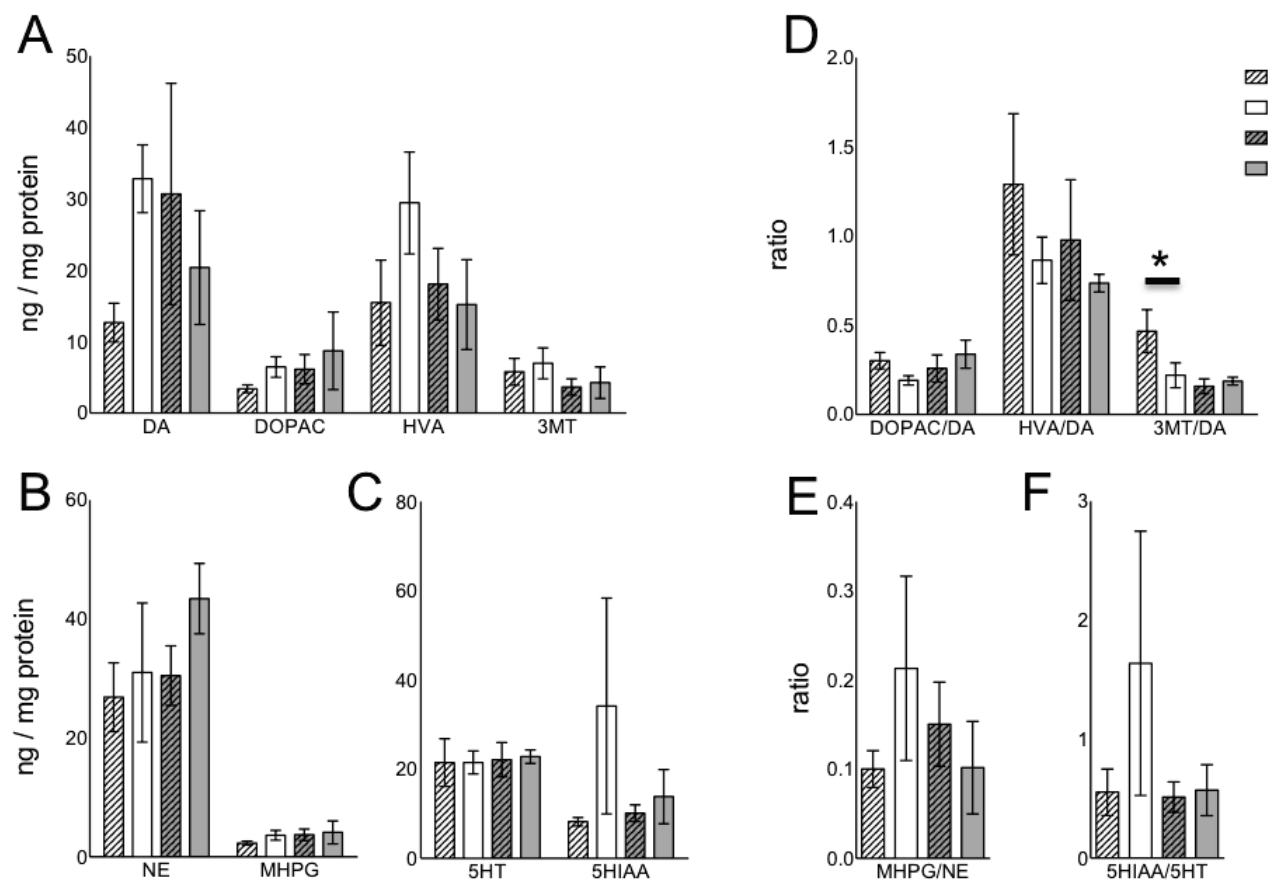

Figure 12. Mean levels (+/- SEM) of DA, NE, 5HT and their metabolites (A-C) and ratio of each monoamine to its metabolites (D-F) in the arcuate nucleus of GHSR-KO and WT rats in Experiment 2B. * denotes a significant strain difference $(\mathrm{p}<0.05)$. 

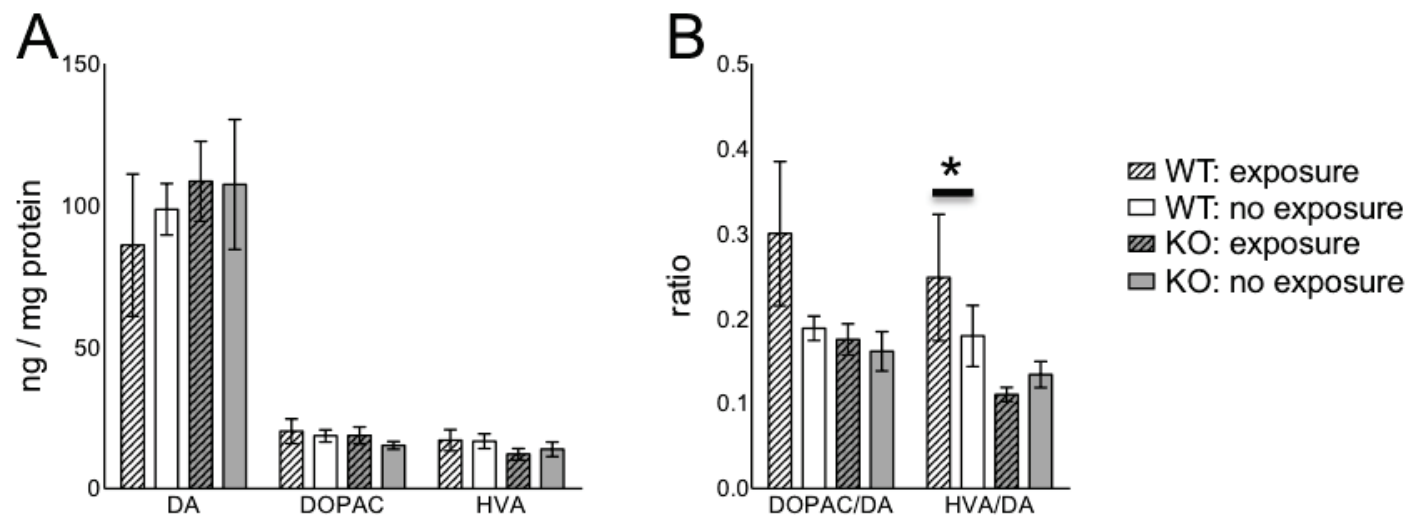

Figure 13. Mean levels (+/- SEM) of DA, NE, 5HT and their metabolites (A-C) and ratio of each monoamine to its metabolites (D-F) in the nucleus accumbens in GHSRKO and WT rats. * denotes a significant strain difference $(\mathrm{p}<0.05)$. 


\section{Discussion}

Operant testing

Experiments 2A and 2B compared WT and GHSR-KO rats' responding in an operant conditioning paradigm using high fat chocolate and sucrose pellets respectively. In both experiments, all rats quickly learned to press the active lever to receive a reward, as evidenced by the rapid escalation in active but not inactive lever presses in the first few days of training. There was no difference between the rats' reinforced and nonreinforced active lever presses (i.e. between the number of pellets earned and the number of responses performed during the time out period) at any point during training. This is different from previous studies using Long Evans rats. Indeed, using a similar reinforcement schedule (fixed ratio of 1, with a 20 or 40 second time out period), Ghitza and colleagues (Ghitza et al., 2007, 2006), as well as Nair and colleagues (Nair, AdamsDeutsch, Pickens, et al., 2009; Nair et al., 2008) found that LE rats show a greater progressive escalation in timeout responses versus reinforced responses, resulting in significant differences between the 2 measures early during training. This pattern of responding is considered to be analogous to the development of compulsive food seeking (Ghitza et al., 2006), and is absent in the FHH WT and GHSR-KO rats. WT and GHSR$\mathrm{KO}$ rats did not differ in terms of active lever presses (both reinforced and nonreinforced) for either high fat chocolate pellets or sucrose pellets.

In the next phase of the experiments, rats were considered to have reached an "extinction criterion" when they pressed the active lever fewer than 11 times over a three hour session during 3 consecutive sessions. GHSR-KO and WT rats trained with high fat chocolate pellets reached this criterion in a comparable amount of time. In contrast, 
GHSR-KO rats trained with sucrose pellets took longer to reach the extinction criterion than WT rats trained in the same conditions. A possible explanation for this perseveration could be that GHSR-KO rats show less behavioural flexibility and inhibitory control than WT rats. Alternatively, strain differences in this measure could reflect strain differences in stress reactivity. Recent evidence suggests that ghrelin acts as a protective factor in times of prolonged stress (reviewed in Chuang \& Zigman, 2010) and that GHSR-KO mice show enhanced depressive and anxiety behaviours in response to stress (Lutter et al., 2008). Stress has also been shown to increase the resistance to extinction in operant paradigms (Highfield et al., 2000; Kupferschmidt, Tribe, \& Erb, 2009). Thus, if rats tested in the current study were under stress during the extinction phase of the paradigm (which could, for instance, originate from the greater level of food restriction as pellets are discontinued - Piazza \& Moal, 1998), and GHSR-KO rats showed a heightened response to this stress, this could manifest as a delay in reaching the extinction criterion.

Food cues and food priming were both effective in reinstating sucrose pellet seeking. In contrast, only food cues were effective in reinstating high fat chocolate pellet seeking. Previous studies suggest that food type can influence reinstatement (Nair, Adams-Deutsch, Epstein, et al., 2009). There is also evidence that cues are most effective at reinstating food seeking than pellet priming, independently of food type (MartínGarcía et al., 2011). Finally, as was seen during training, there were no strain differences during reinstatement for either sucrose or high fat chocolate pellet seeking.

The fact that there were no strain differences in food seeking during training and reinstatement for sucrose and high fat chocolate pellets was an unexpected result. This finding may indicate that ghrelin is not involved in operant responding for palatable 
foods or the reinstatement of food seeking. This, however, is unlikely, as previous studies have shown that peripheral (i.p.) and central (i.c.v., intra-hippocampal, and intra-VTA) ghrelin administration increases breakpoints (i.e. the maximum number of responses an animal will perform to receive a single reward when the requirements for each reward are progressively increased) and overall lever pressing for heroin, as well as sucrose, chocolate, banana and high fat pellets (Kanoski et al., 2013; King et al., 2011; Maric et al., 2012; Overduin et al., 2012; Perello et al., 2010; Skibicka et al., 2011; Skibicka, Hansson, et al., 2012; Skibicka, Shirazi, et al., 2012; Weinberg et al., 2011). Similarly, peripheral (i.p.) and central (i.c.v. and intra-VTA) administration of GHSR antagonists reduce operant breakpoints and overall lever pressing for heroin, ethanol, sucrose solution as well as chocolate and sucrose pellets (King et al., 2011; Landgren et al., 2012; Landgren, Simms, et al., 2011; Maric et al., 2012; Skibicka et al., 2011; Skibicka, Hansson, et al., 2012). Notably however, continuous schedules of reinforcement such as the one used in this experiment are less sensitive to motivational factors than progressive ratio schedules (Sanchis-Segura \& Spanagel, 2006). In addition, although there is currently no direct evidence linking ghrelin and the reinstatement of food seeking, Tessari and colleagues (2007) found that endogenous ghrelin levels are positively correlated with the reinstatement of cocaine seeking elicited by cocaine-associated cues. An alternate explanation for the current findings is that, over their lifetime, GSHR-KO rats developed compensatory mechanisms that limit the effects of absent ghrelin signalling. Such mechanisms are suggested by the fact that several studies report discrepancies between pharmacological and genetic models of ghrelin signalling disruptions (i.e. differences between adult mice treated with a GHSR antagonist and age- 
matched mice lacking the gene for ghrelin or GHSR) (Abizaid et al., 2006; Egecioglu et al., 2010; Jerlhag, Egecioglu, et al., 2011; Perello et al., 2010; Walker et al., 2012). Finally, it is important to note that response rates observed during training in the current study are substantially lower than those observed in studies using Long Evans rats (Ghitza et al., 2007, 2006; Nair, Adams-Deutsch, Pickens, et al., 2009; Nair et al., 2008). It is possible that lower responding in the FHH strain contributed to masking the effects of ghrelin signalling in this paradigm (floor effect).

Brain monoamines

The monoamines play an important modulatory role in structures of the reward system innervated by the VTA. Compared to WT rats, GHSR-KO rats had reduced monoamine levels (HVA, 5HT and NE) in the prefrontal cortex (PFC) and lower dopamine utilization in the arcuate and accumbens nuclei. The PFC supports many higher order functions including inhibitory response control. Rats with PFC lesions show perseverations in operant conditioning tasks when the responses are no longer reinforced, i.e. they take longer to show extinction (reviewed in Kolb, 1984). The monoamines affect the expression of PFC-mediated behaviours. For instances, drugs that increase NE and DA in the PFC improve performance on inhibitory response control tasks and decrease the incidence of impulsive behaviours (Bari et al., 2011; Robbins \& Arnsten, 2010). 5HT receptor antagonists and 5HT depletion in the PFC increase perseverations in reversal tasks, i.e. learning tasks that require behavioural changes in response to changes in stimulus-reward contingencies (Boulougouris, Glennon, \& Robbins, 2008; Clarke, Dalley, Crofts, Robbins, \& Roberts, 2004; reviewed in Eagle \& Baunez, 2010). Therefore, it is possible that lower PFC monoamine levels in the GHSR-KO rats relative 
to the WT rats contributed to their delay in reaching the extinction criterion in the sucrose operant responding paradigm. Reduced DA utilization in the GHSR-KO rats relative to WT rats is consistent with the fact that systemic ghrelin administration increases the ratio of DOPAC to DA in outbred rats (Abizaid et al., 2006).

Exposure to the operant conditioning box increased NE metabolism and decreased 5HT metabolism in the amygdala of WT and GHSR-KO rats. The amygdala is sensitive to emotionally relevant stimuli and plays a role in associative learning (reviewed in Gallagher \& Holland, 1994). Specifically, the BLA receives sensory information about reinforcers and is important for their association with neutral events that predict them such as, in the current study, the contextual cues of the operant conditioning box (reviewed in Everitt et al., 2003). The current findings are consistent with an increase in BLA activity following the presentation of reward cues as 5HT was previously shown to have an inhibitory action in the amygdala (Stutzmann, McEwen, \& LeDoux, 1998), and unexpected positive events increase NE release in the BLA (Young \& Williams, 2010).

Exposure to the operant conditioning box reduced amygdala 5HT and HVA levels in GHSR-KO but not WT rats. Although the functional significance of these findings is unclear, it is interesting that the BLA is necessary for the association between objects and their current reinforcing value. For instance, rats with BLA lesions are able to form associations between palatable foods and their conditioned stimuli (CS), but cannot adapt to changes in the predictive value of the CS (i.e. if the CS is subsequently "re-paired" with a new outcome) (Baxter \& Murray, 2002). In Experiment 2B, GHSR-KO rats were slow to adapt to a change in reinforcement schedule during extinction. It is possible that altered amygdala functioning contributed to this effect. 


\section{Conclusion}

In this experiment, food cues, but not food priming, elicited the reinstatement of high fat chocolate pellet seeking whereas both stimuli elicited the reinstatement of sucrose pellet seeking. This suggests that food cues are more effective reinstatement stimuli than food priming. GHSR-KO and WT rats performed similarly during training and reinstatement using both types of reinforcers. In contrast, GHSR-KO took longer to reach an extinction criterion when sucrose pellets were discontinued. This finding may partly be explained by strain differences in prefrontal monoamine levels. 


\section{Chapter 5: The role of ghrelin in reinstatement -}

\section{conditioned place preference}

Hypothesis 2: ghrelin facilitates the reinstatement of palatable food seeking that is triggered by food priming and food associated cues.

Aim 4: to compare GHSR-KO and WT rats in the conditioned place preference version of the reinstatement test.

\section{Introduction}

The experiments performed in chapter 4 revealed that WT and GHSR-KO rats display comparable levels of food seeking reinstatement after an extinction period in an operant conditioning model. This was observed independently of food type (sucrose vs. high fat chocolate pellets) and reinstatement stimulus (cue-induced vs. food priminginduced). Although it is possible that ghrelin signalling is not involved in food reinstatement, a number of observations suggest that this is not necessarily the case. For instance, the experiments found no strain differences during training - this is inconsistent with several studies that implicate ghrelin in operant responding for palatable foods (Davis et al., 2012; Kanoski et al., 2013; King et al., 2011; Overduin et al., 2012; Perello et al., 2010; Skibicka et al., 2011; Weinberg et al., 2011). In addition, WT and GHSR-KO rats had response rates that were considerably lower than those reported in other rat strains, using similar pellets and reinforcement schedules (Ghitza et al., 2007, 2006; Nair, Adams-Deutsch, Pickens, et al., 2009; Nair et al., 2008). These observations may indicate that compared to other rat strains, the FHH rats used for the generation of the WT and 
GHSR-KO strains are less responsive to the rewarding effects of food and/or less willing to work for it. Under such circumstances, any strain differences due to ghrelin signalling may be undetectable. For these reasons, the next experiment compared GHSR-KO and WT rats' reinstatement in a different experimental paradigm, the conditioned place preference $(\mathrm{CPP})$ reinstatement test.

As mentioned in chapter 1, the CPP test is used to infer the rewarding properties of substances based on the amount of time an animal spends in a context previously associated with the substance (Tzschentke, 2007). As in the operant conditioning model, an established response (i.e. place preference) can be extinguished via repeated presentations of the context in the absence of the substance and subsequently reinstated. Effective reinstatement stimuli in the CPP model are the same as in the operant conditioning model: re-exposure to the substance, exposure to conditioned stimuli associated with the substance, and stress (Tzschentke, 2007).

The CPP reinstatement model offers 2 major advantages over the operant conditioning reinstatement model that make it particularly attractive for the study of FHH GHSR-KO and WT rats. First, the CPP task does not have a strong motor component. If FHH rats are generally less motivated to perform motor responses to obtain rewards than other rat strains, reducing the motoric demands of the task may help uncover differences in other types of reward behaviours between GHSR-KO and WT rats. In addition, the CPP model allows the use of a wide variety of rewards as opposed to only those who fit the size and shape specifications of the operant conditioning apparatus.

In the current study, we assessed the role of ghrelin in a CPP paradigm using chocolate chip cookie dough as a reward. Specifically, we planned to compare 1) GHSR- 
KO and WT rats' propensity to develop a place preference to this reward, 2) the rate at which their place preferences extinguished upon reward discontinuation, and 3) whether or not food priming could reinstate their place preference following extinction. Unexpectedly, repeated presentations of the testing apparatus in the absence of cookie dough did not result in the extinction of a conditioned place preference in the rats, which made it impossible to look at reinstatement. We therefore adapted our experimental protocol as to examine the strength of GHSR-KO and WT rats' place preference following manipulations of the testing environment expected to oppose the expression of the place preference.

\section{Methods: Experiment 3}

Baseline measures: Nineteen rats $(9 \mathrm{KO}+10 \mathrm{WT})$ were used in experiment 3. Baseline food intake was measured daily for 4 days before the start of the study. All sessions began approximately 2 hours after the start of the light phase. Prior to place preference conditioning, rats underwent a 20 -minutes pre-conditioning session during which they had access to both sides. The number of seconds spent in each compartment was recorded. Rats were also given cookie dough overnight on 2 occasions on nonconsecutive nights ( 25 grams the first night and 50 grams the second night).

Place preference conditioning and CPP testing: Throughout the course of the experiment, rats underwent 3 conditioning rounds and 5 CPP tests. During conditioning, the CPP chambers were separated and rats were placed in the white and dark chambers for 30-minutes sessions on alternating days. One minute after being placed there, a small ceramic plate containing 5 grams of either cookie dough (chocolate chips, Pillsbury) or regular chow was put in the chamber. We used a biased CPP protocol - i.e. the rewarding 
food (cookie dough) and regular chow were consistently paired with the least and most preferred chamber (as determined by the preconditioning session), respectively.

During the first conditioning round (18 sessions), rats were maintained on an adlib feeding schedule and conditioned in bright light. During the second round (20 sessions), rats were maintained on a restricted feeding schedule (access to food limited to 4 hours per day, starting after the conditioning session) and conditioned in bright light. During the third round ( 8 sessions), rats were maintained on a restricted feeding schedule and conditioned in dim light. CPP tests took place at the following time points: in the middle and at the end of the $1^{\text {st }}$ conditioning round, in the middle and at the end of the $2^{\text {nd }}$ conditioning round, and at the end of the $3^{\text {rd }}$ conditioning round (see figure 14). The first 4 tests were done in bright light conditions, and the $5^{\text {th }}$ test was done in dim light conditions. During the tests, rats were placed in the CPP boxes without any food and had access to both chambers for 20 minutes. The number of seconds spent in each chamber was recorded.

Extinction and post-extinction CPP tests: Rats were returned to an adlib-feeding regimen immediately after the last CPP tests. Extinction began 3 days following this. During this phase, rats were placed in the CPP boxes with no food and with access to both chambers for 20 minutes every day until they reached an extinction criterion (defined as less than $15 \%$ of the time spent in the cookie dough-paired chamber on 3 consecutive days) or for a maximum of 20 days (whichever came first).

One week after the end of extinction, each rat received an additional 2 CPP tests, separated by 48 hours. In one version of the test, rats were tested in dim light, 20 minutes after receiving 2 grams of cookie dough ("priming" condition). In the other version, rats 
were tested in bright light conditions ("lights" condition). Half of the rats received the priming condition first, and the other half received the lights condition first. In both cases, the number of seconds spent in each compartment was recorded. 


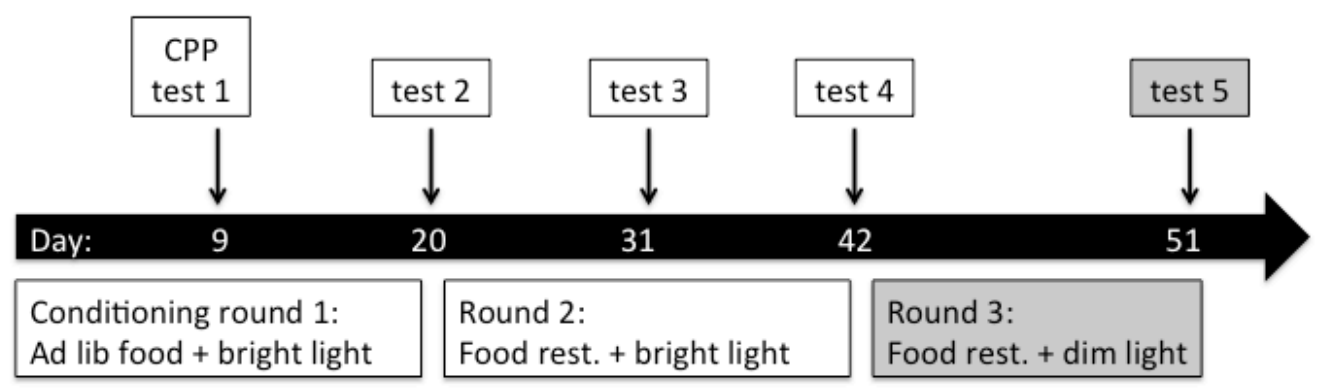

Figure 14. Timeline of CPP tests in Experiment 3. 


\section{Results: Experiment 3}

Baseline measures: GHSR-KO and WT rats ate comparable amounts of chow at baseline (average of 4 days: $[\mathrm{t}(17)=1.686, \mathrm{p}=0.110]$ ) and of cookie dough when it was given over night (Figure 15). When 25 grams of cookie dough were given, the majority of rats ate it all; when 50 grams were given, GHSR-KO and WT rats ate an average of 28.67 and 28.20 grams respectively $[\mathrm{t}(17)=2.0259, \mathrm{p}=0.799]$. 

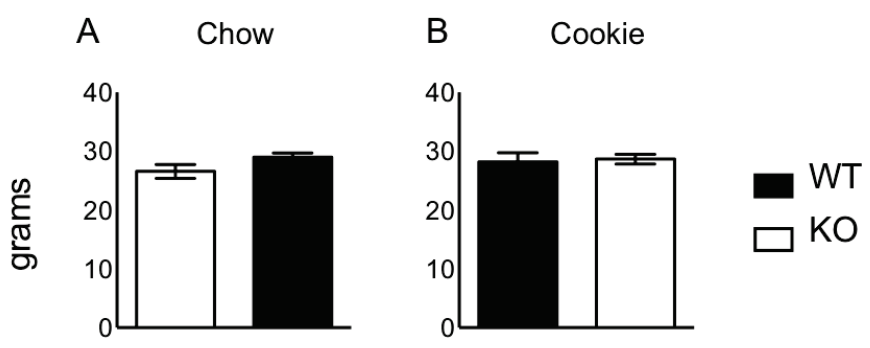

Figure 15. Baseline daily chow intake (average of 4 days) and overnight cookie dough intake ( ${ }^{\text {nd }}$ night) in GHSR-KO and WT rats in Experiment 3 (NS). 
Conditioning and CPP testing: A repeated measure ANOVA revealed that prior to conditioning, rats spent significantly more time in the dark compartment than the white one $[F(1,17)=390.452, p<0.001]$ (Figure 16A). Since this pattern was clearly apparent for each rat, cookie dough exposure during conditioning was paired to the white compartment exclusively. We used a series of repeated measure ANOVAs to compare time spent in the white compartment during CPP tests versus the pre-conditioning sessions in GHSR-KO and WT rats. The analyses revealed no effects of strain or session and no interaction of the 2 factors for the $1^{\text {st }}, 2^{\text {nd }}$ and $4^{\text {th }} \mathrm{CPP}$ tests (all $\mathrm{p}$ values $>0.05$ ). In contrast, rats spent more time in the white compartment during the $3^{\text {rd }} \mathrm{CPP}$ test than during the preconditioning session $[\mathrm{F}(1,17)=11.480, \mathrm{p}=0.003]$. This pattern was even more pronounced in the $5^{\text {th }} \mathrm{CPP}$ test $[\mathrm{F}(1,17)=68.626, \mathrm{p}<0.001]$, indicating that rats had developed a place preference for the cookie dough-paired compartment (Figure 16B); this preference was similar in GHSR-KO and WT rats (all $\mathrm{p}$ values $>0.05$ ). 

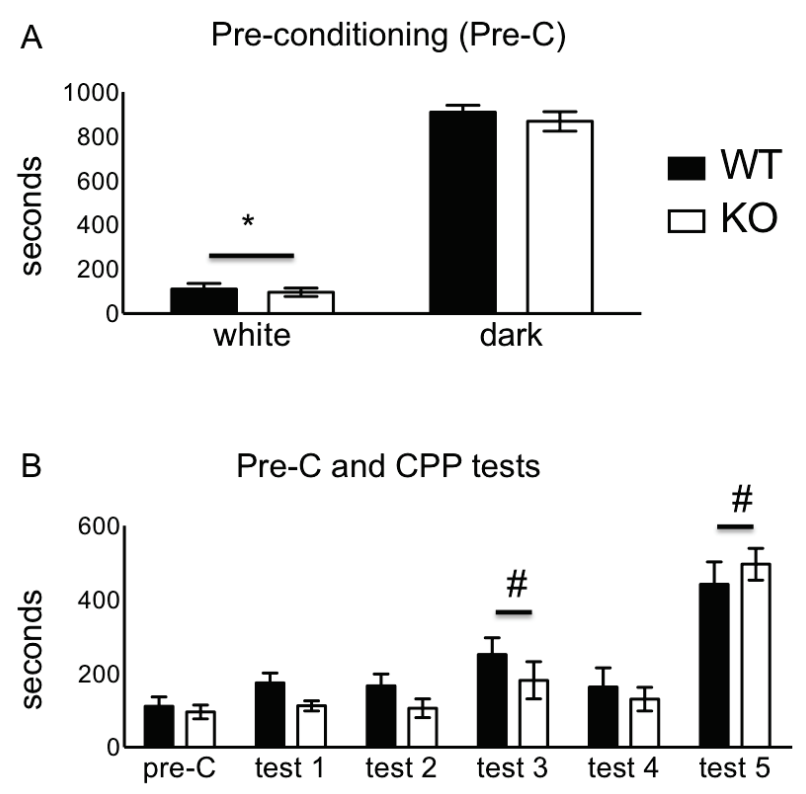

Figure 16. A. Time spent in the white and dark compartments during pre-conditioning in GHSR-KO and WT rats in Experiment 3. * denotes a significant difference between the time spent in each compartment $(p<0.05)$ B. Time spent in white compartment during pre-conditioning and $5 \mathrm{CPP}$ tests in GHSR-KO and WT rats. \# denotes a significant difference between the time spent in the white compartment during CPP testing relative to pre-conditioning levels $(\mathrm{p}<0.05)$. 
Extinction sessions: A repeated measure ANOVA compared the time spent in the white compartment during an "extinction" phase, consisting of repeated exposures to the entire CPP apparatus with no reward on either side. We predicted that over time, rats' preference for the white compartment would extinguish and that they would spend less time in it. The analysis revealed a significant main effect of test session $[F(1,17) 1.851, p$ $=0.017]$. A follow up paired $\mathrm{t}$ test comparing the first and last sessions revealed that, surprisingly, rats spent more time in the white compartment as time went by $[\mathrm{t}(18)=$ $3.050, \mathrm{p}=0.007]$. There was also a significant strain by session interaction $[\mathrm{F}(1,17)=$ 1.699, $\mathrm{p}=0.035]$. Paired $\mathrm{t}$ tests comparing the first and last sessions for each strain separately revealed that only WT rats significantly increased the time they spent in the white chamber between these 2 time points (WT rats: $[t(9)=-5.241, \mathrm{p}=0.001]$; KO rats: $[\mathrm{t}(8)=-0.758, \mathrm{p}=0.470])($ Figure 17A)

Post-extinction tests: An additional 2 CPP tests were performed at the end of the experiment: one was performed under bright light conditions (as during the preconditioning session and the first 2 conditioning rounds), and the other after cookie dough priming. A series of paired $t$ tests revealed that cookie dough priming did not affect the amount of time rats spent in the white compartment, relative to the last CPP test and last "extinction" session ( $\mathrm{p}>0.05$ in both cases). In contrast, performing the test in bright light reduced the time rats spent in the white compartment to pre-conditioning levels ( $p<0.05$ relative to last CPP test and last extinction session; $p>0.05$ relative to pre-conditioning session). Whereas GHSR-KO and WT rats were indistinguishable in the cookie dough priming condition $[\mathrm{t}(17)=-1.256, \mathrm{p}=0.266]$, WT rats spent significantly 
more time in the white compartment than GHSR-KO rats when bright lights were on $[\mathrm{t}(17)=-2.249, \mathrm{p}=0.038]($ Figure 17B $)$

Repeated measure ANOVAs comparing place preference during the last extinction day and each additional testing day were performed to determine if strain differences during the final tests were indeed due to the bright lights and/or cookie dough or if these effects carried over from the extinction phase. The analysis performed for the cookie dough priming condition revealed a significant strain effect $[\mathrm{F}(1,17)=6.435, \mathrm{p}=$ $0.021]$, but no effect of session or session by strain interaction $(p>0.05)$. In light of the fact that there was a strain difference in place preference on the last day of extinction but not after cookie dough priming, it is likely that this effect was driven by the last extinction session. Finally, the analysis performed for the bright lights condition revealed a significant strain effect $[\mathrm{F}(1,17)=6.621, \mathrm{p}=0.020]$, a significant session effect $[\mathrm{F}(1,17)=144.704, \mathrm{p}<0.001]$, but no strain by session interaction $(\mathrm{p}>0.05)$. Taken together, these results indicate that WT rats displayed a stronger food-induced place preference than GHSR-KO rats, bright lights diminished the amount of time rats spent in the white compartment, but bright lights did not differentially affect GHSR-KO and WT rats. 

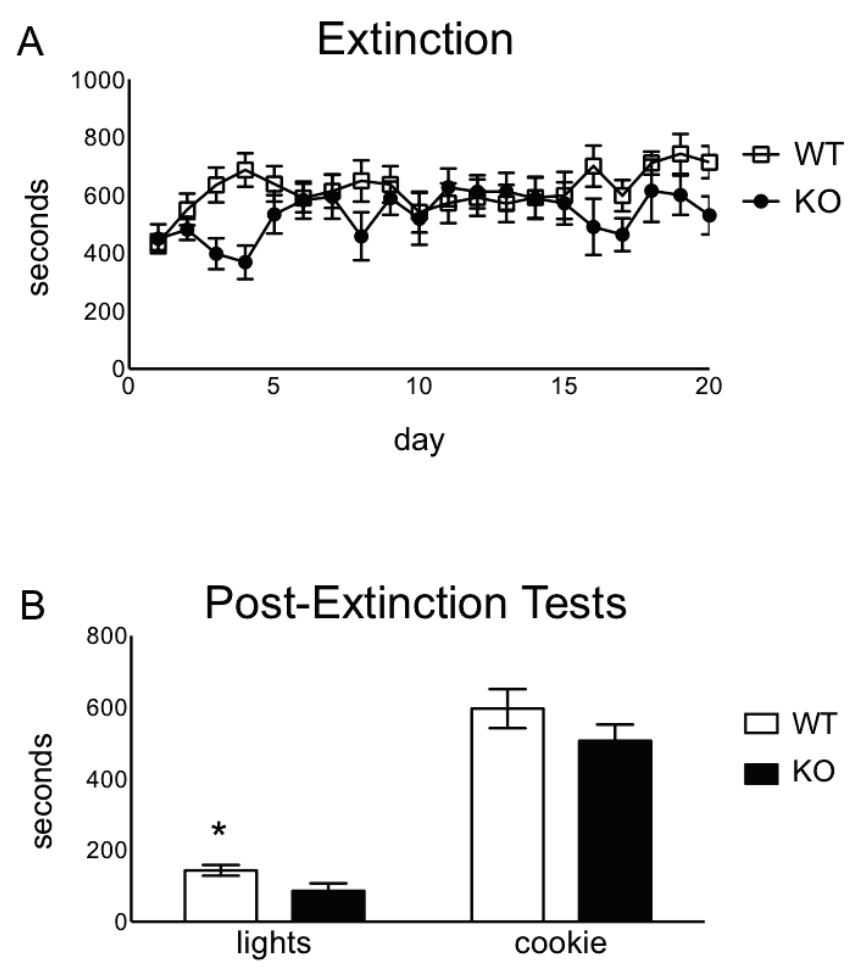

Figure 17. Experiment 3: A. Time spent in the white compartment over 20 nonreinforced CPP tests. WT rats increased the time they spent in the white compartment during this period $(p=0.001)$ whereas GHSR-KO rats did not $(p=0.470)$. B. Time spent in the white compartment during 2 CPP tests at the end of Experiment 3.* denotes $\mathrm{p}<0.05$. 


\section{Discussion}

The main finding of the current study is that GHSR-KO and WT rats differentially responded to the discontinuation of cookie dough in the CPP test. Repeated CPP testing (in the absence of cookie dough) did not result in the extinction of the established place preference in either strain. WT rats' CPP scores, but not KO's CPP scores, increased over the course of this "extinction" phase. Some operant and CPP studies examining extinction also report an increase in drug seeking (lever pressing or CPP expression) over time. This phenomenon, termed incubation of drug craving, is thought to be the natural outcome of withdrawal (Grimm, Hope, Wise, \& Shaham, 2001; Li et al., 2008). If incubation took place in the current experiment, then it may be inferred from the present data that the absence of ghrelin signaling is protective against this phenomenon. Consistent with this hypothesis are the findings that 1) the prefrontal cortex is involved in the incubation of cocaine craving (Koya et al., 2009) and is functionally different in GHSR-KO and WT rats (Experiment 2B), and 2) ghrelin levels are positively correlated with alcohol craving in alcoholics (Koopmann et al., 2012; Leggio et al., 2014).

Nevertheless, the lack of extinction in this study is inconsistent with the extinction results in the operant conditioning paradigm (Experiments $2 \mathrm{~A}$ and $2 \mathrm{~B}$ ). It is important, however, that operant conditioning and CPP examine different types of reward behaviors; whereas operant conditioning assesses the primary reinforcing properties of a substance, CPP assesses the incentive value of conditioned stimuli associated with that substance (reviewed in Aguilar, Rodríguez-Arias, \& Miñarro, 2009). It is therefore possible that the processes sustaining extinction in these 2 paradigms also differ. In addition, it is possible 
that the active component inherent to operant responding makes this behavior easier to extinguish.

Further, different extinction paradigms in the CPP test have different outcomes. In general, CPP extinction can be conducted in 2 ways. In explicit extinction training, the newly preferred compartment is repeatedly paired with a neutral stimulus. After a few exposures (and pairings) to that chamber, a last CPP test is performed. In the second version of extinction, animals receive unrewarded CPP tests (i.e. with full access to both compartments, in the absence of the reinforcer) daily until the place preference is no longer apparent. In the current study, the latter approach was selected to permit the detection of strain differences in the number of days required to reach an extinction criterion. Although both techniques have previously been shown to result in the extinction of place preference, explicit extinction training accomplishes this in a shorter time period (Mueller \& Stewart, 2000). Indeed, some studies suggest that in the absence of explicit extinction training, conditioned stimuli maintain their ability to direct behavior in the CPP paradigm (Mueller, Perdikaris, \& Stewart, 2002), and higher numbers of pairings during the conditioning phase result in more persistent preferences (SanchisSegura \& Spanagel, 2006).

As observed in study 1, GHSR-KO and WT rats ate similar amounts of chow at baseline. GHSR-KO and WT rats also ate similar amounts of cookie dough when it was given over night. While this finding may appear inconsistent with the results of Experiment 1A, there is an important difference between the 2 experiments. Here, the rats ate cookie dough during the dark phase (i.e. at their usual meal time) and presumably, although this was not measured, instead of their regular chow. This differs from 
Experiment $1 \mathrm{~A}$, where rats ate cookie dough after a meal during which they consumed the majority of their baseline daily intake. In fact, similar meal sizes in GHSR-KO and WT rats are consistent with previous observations from experiments conducted to date (Experiments 1A and 1B; Bülbül et al., 2011).

All rats displayed an initial preference for the dark compartment of the CPP apparatus. This is likely due to the fact that brightly lit environments are unpleasant for rodents and reduce exploratory behaviors (Costall, Jones, Kelly, Naylor, \& Tomkins, 1989). The first 2 conditioning rounds, performed in a brightly lit room under adlib (round 1) and food restriction (round 2) conditions did not result in a conditioned place preference for the cookie dough-paired white chamber in 3 out of 4 CPP tests. In contrast, the third conditioning round, performed in a dimly lit environment resulted in a marked preference for the white chamber. While it cannot be ruled out that the development of CPP after the $3^{\text {rd }}$ conditioning round is simply due to more conditioning, a facilitatory effect of dim light conditions has previously been observed in similar studies (Roma \& Riley, 2005) and is likely at play in the current experiment as well.

GHSR-KO and WT rats performed similarly in all 5 CPP tests. This is inconsistent with a number of studies demonstrating reduced CPP to ethanol and a high fat diet in GHSR-KO and ghrelin-KO mice relative to their WT counterparts (Bahi et al., 2013; Jerlhag et al., 2009; Perello et al., 2010). The results also contrast with studies that show enhanced CPP to cocaine and a high fat diet in mice and rats following systemic ghrelin administration (Davis et al., 2007; Perello et al., 2010) and reduced CPP to chocolate, ethanol, cocaine, amphetamines and nicotine after systemic and central GHSR antagonist treatments (Bahi et al., 2013; Jerlhag et al., 2009, 2010; Jerlhag \& Engel, 
2011; Skibicka, Hansson, et al., 2012). As in the operant conditioning paradigm, a possible explanation for the discrepancies between our results and others' may lie in the background strain used to generate the current model. If CPP scores were generally low in GHSR-KO and WT rats, any strain differences due to ghrelin signaling may have been difficult to detect. Alternatively, compensatory mechanisms in the GHSR-KO strain may have diminished differences between the 2 strains in the CPP task.

The lack of extinction upon cookie dough discontinuation prevented the examination of reinstatement in GHSR-KO and WT rats. We therefore adapted our protocol to include 2 additional and final manipulations. The first manipulation sought to determine if the use of a classic reinstatement stimulus would further increase CPP expression. The results indicated that cookie dough priming prior to an extinction session/CPP test did not affect CPP scores. In the second manipulation, we examined the effect of bright light conditions on place preference. The choice of this manipulation was based on two observations: 1) bright lights act as a stressor in rodents (Strekalova, Spanagel, Dolgov, \& Bartsch, 2005) and may interfere with the development of a conditioned place preference (current study), and 2) ghrelin signaling affects the behavioral response to stress and may act as a protective factor in stressful situations (Chuang \& Zigman, 2010). Thus, the bright light condition tested the hypothesis that the final expression of CPP is affected by both stress and motivational factors and that ghrelin impacts this relationship. The results did not support the hypothesis. Although bright lights reduced the time spent in the white chamber in GHSR-KO rats more than in WT rats, a more detailed analysis revealed that the strain differences preempted this condition and that the lights did not differentially affect the 2 strains. 


\section{Conclusion}

In this experiment, GHSR-KO and WT rats performed similarly in a CPP task using cookie dough as a reinforcer. As observed in chapter 4, strain differences were only noticed when the reinforcer was discontinued. Notably, whereas reinforcer discontinuation led to extinction of the lever pressing response in operant conditioning, it did not extinguish a place preference in the current experiment. In fact, WT rats' preference for the cookie dough-paired compartment increased during the course of an “extinction” period. GHSR-KO rats appeared protected from this sensitization. 


\section{Chapter 6: The role of intra-VTA ghrelin in}

\section{reinstatement}

Hypothesis 3: ghrelin facilitates the reinstatement to palatable food seeking that is triggered by food priming, food associated cues and stress by acting at the level of the VTA.

Aim 5: to assess operant conditioning reinstatement in Long Evans rats receiving either ghrelin or a GHSR antagonist chronically into the VTA.

\section{Introduction}

Data obtained so far suggest that the GHSR-KO rats are a useful model to study some reward-related behaviours but not others. Consequently, the experiments described in Chapter 6 were performed using ghrelin- and GHSR antagonist-treated Long Evans (LE) rats in order to characterize ghrelin's role in the reinstatement of palatable food seeking. As in Chapter 4, we used an operant conditioning model consisting of 3 phases: training, extinction, and reinstatement. Rats were implanted sub-cutaneous mini-pumps delivering ghrelin, a GHSR antagonist or vehicle solution continuously into the VTA between the extinction and the reinstatement phases. The choice of the pharmacological treatment administration route was based on the presence of GHSRs within this node of the reward system and evidence suggesting the VTA plays a role in the reinstatement of both cocaine and heroin seeking (Abizaid et al., 2006; Bossert, Liu, Lu, \& Shaham, 2004; Guan et al., 1997; Stewart, 1984; Wang, You, Rice, \& Wise, 2007; Wang et al., 2009; Zigman et al., 2006). 
Using this model, the following experiments examined the effects of VTA GHSR manipulations on reinstatement of food seeking (sucrose and high fat chocolate pellets) elicited by food priming, food associated cues, a mild stressor (an overnight fast), and the pharmacological stressor yohimbine. Yohimbine is frequently used in reinstatement studies because it induces stress- and anxiety-like behaviours in humans and rodents alike and reliably triggers reinstatement in both drug and food seeking studies (reviewed in See \& Waters, 2011).

The use of LE rats as opposed to GHSR-KO and WT rats required one additional modification to the experimental protocol described in Chapter 4. In order to limit the effects of endogenous ghrelin levels, which naturally rise in times of negative energy balance, at GHSRs located outside of the mesolimbic system, reinstatement was tested in animals that recently had unlimited access to regular food.

\section{Methods: Experiment 4 (A \& B)}

Experiment 4A:

Autoshaping, training \& Extinction: Thirty-six male Long Evans rats (216-375 grams, average $=283$ grams) were used in Experiment 4A. Testing began 5 days after the start of food restriction. All animals were subjected to a single 3-hour long autoshaping session during which high-fat chocolate pellets were delivered every 5 minutes. Each pellet delivery was accompanied by a 2 second light/tone stimulus. Starting on the next day, rats were trained for 3 hours every second day, for 24 days (12 training sessions). During training, pressing on the active lever resulted in the delivery of a pellet and exposure to the food cue (light/tone stimulus). Each pellet delivery was followed by a 20 second timeout period, during which pressing on the lever had no consequence. 
Similarly, pressing on the inactive lever at any point had no programmed consequence. Training sessions were conducted during the morning (9:00AM-12:00PM) or afternoon (1:00PM-4:00PM), which alternated for each rat. At the start of the first 5 sessions, 3 pellets were placed on the active lever in order to facilitate acquisition. On training days, rats were fed their maintenance food ration 1 hour after the conditioning session. On "off" days, rats were fed 1 hour after the start of the dark phase.

One day after the completion of the training phase, rats were exposed to a series of 3-hour extinction sessions, during which lever pressing (active or inactive) had no programmed consequence. Rats received daily extinction sessions until they reached the extinction criterion, defined as pressing on the active lever less than 30 times over 3 hours on 3 consecutive days. Rats were returned to an adlib-feeding schedule until the day of surgery.

Surgery and recovery: Stereotaxic surgery was performed 1 to 6 days after the end of the extinction period. Isoflurane and oxygen were used to anesthetise rats for surgery. A cannula (Plastics One, 3300P-SPC), connected to a mini-pump (Alzet, model 2002) by a $4 \mathrm{~cm}$ long vinyl tube (Plastics One, C312-VT) was then implanted into the VTA. Coordinates for implantation were $5.3 \mathrm{~mm}$ posterior to bregma, $2 \mathrm{~mm}$ lateral to the midline, and 7.6mm below the skull surface (Paxinos \& Watson, 1998). The cannula was anchored into position by dental acrylic placed around 3 stainless steel screws embedded within the skull. The mini-pumps, implanted subcutaneously between the shoulder blades, delivered saline, ghrelin (10nmol; Pi Protemics, PI-G-01), or a ghrelin receptor antagonist, [D-Lys-3]-GHRP-6 (200nmol; Peptides International, OGH-3656-PI) into the VTA at a rate of $0.5 \mu \mathrm{L} / \mathrm{hr}$ for 14 days. The wound was closed with sutures. Following 
surgery, rats received a subcutaneous injection of meloxicam (Metacam ${ }^{\circledR}, 0.1 \mathrm{~mL}$ ) to minimize pain and discomfort and were placed in a recovery cage until normal behavioural activity was observed.

Recovering rats were fed mashed food (chow and water mixture) for 1 day following the surgery, and then returned to an ad lib feeding schedule for a period of 4 days, during which food intake was recorded daily. Rats were then returned to a restricted feeding regimen for 2 days, and tested for reinstatement of food seeking.

Reinstatement: Food seeking (i.e. active lever pressing) was reinstated by 1) preexposure to the food pellets (food priming), 2) contingent presentations of the light-tone food cue upon lever pressing, or 3) an overnight fast. Rats also received an additional extinction session, during which no reinstatement stimulus was used. All rats were exposed to the 4 conditions in a counterbalanced order. In the food priming condition, 5 pellets were delivered in a non-contingent fashion at the start of the session. In the food cue condition, rats were presented with the tone/light cue 3 times at the start of the session, and upon all active lever presses during the session. In the overnight fast condition, food was removed from the home cage approximately 18 hours before the session. Forty-eight hours separated each reinstatement session. With the exception of the tone/light stimulus presentations in the food cue condition, the reinstatement sessions themselves were identical to extinction sessions. Locomotion during these sessions was recorded using an infrared activity monitor mounted on the ceiling of the cage (Coulbourn Instruments ${ }^{\circledR}$ ). 
Rats were euthanized 24 hours following the last reinstatement session. Brain sections containing the cannula tract were then mounted on gelatin-coated slides and stained with cresyl violet to ensure accurate placement of the cannula tip within the VTA. Experiment 4B:

Sixteen Long Evans rats (334-381 grams, average = 357 grams) were used in experiment 4B. Acclimation, autoshaping, training, extinction and surgery proceeded as described in experiment 4A, with the exception that sucrose pellets were used instead of high fat chocolate pellets. During surgery, 8 rats were implanted [D-Lys-3]-GHRP-6filled cannulae and 8 rats were implanted saline-filled cannulae. The cannulae delivered [D-Lys-3]-GHRP-6 or saline into the VTA at a rate of $0.5 \mathrm{uL} / \mathrm{hr}$ for 14 days. Recovery occurred as in experiment $4 \mathrm{~A}$.

Reinstatement of food seeking was assessed following a single yohimbine (2 $\mathrm{mg} / \mathrm{kg}$ in $0.5 \mathrm{mg} / \mathrm{kg}$ distilled water; yohimbine hydrochloride, Sigma, Y-3125) or vehicle $(0.5 \mathrm{mg} / \mathrm{kg}$ distilled water) intra-peritoneal injection. All rats were exposed to the 2 conditions in a counterbalanced order and 48 hours separated each reinstatement session. Injections took place 30 minutes prior to the start of the sessions, which were identical to extinction sessions. Locomotor activity was recorded throughout each session.

\section{Results: Experiment 4 (A \& B)}

Experiment 4A:

Pre and post surgery weights: Rats' weights over the course of the experiment fluctuated as a function of chow and pellet availability. A series of paired t tests revealed that, at the outset of the study, rats' weights decreased after 5 days of food restriction $[\mathrm{t}(35)=7.436, \mathrm{p}<0.001, \mathrm{~d}=0.167]$. Weights then increased over the course of training 
$[\mathrm{t}(35)=-7.014, \mathrm{p}<0.001,=0.390]$ and decreased over the course of extinction $[\mathrm{t}(35)=$ $5.769, \mathrm{p}<0.001, \mathrm{~d}=0.167]$, presumably as a result of pellet availability during operant conditioning. This was also the case when only looking at the 18 rats subsequently tested for reinstatement (see below; $\mathrm{p}<0.05$ in all cases) (Figure 18). In these rats, body weights were significantly higher at the start and end of the reinstatement phase than at baseline and after extinction ( $p<0.05$ in both cases), presumably as a result of unlimited access to regular chow between extinction and reinstatement. 


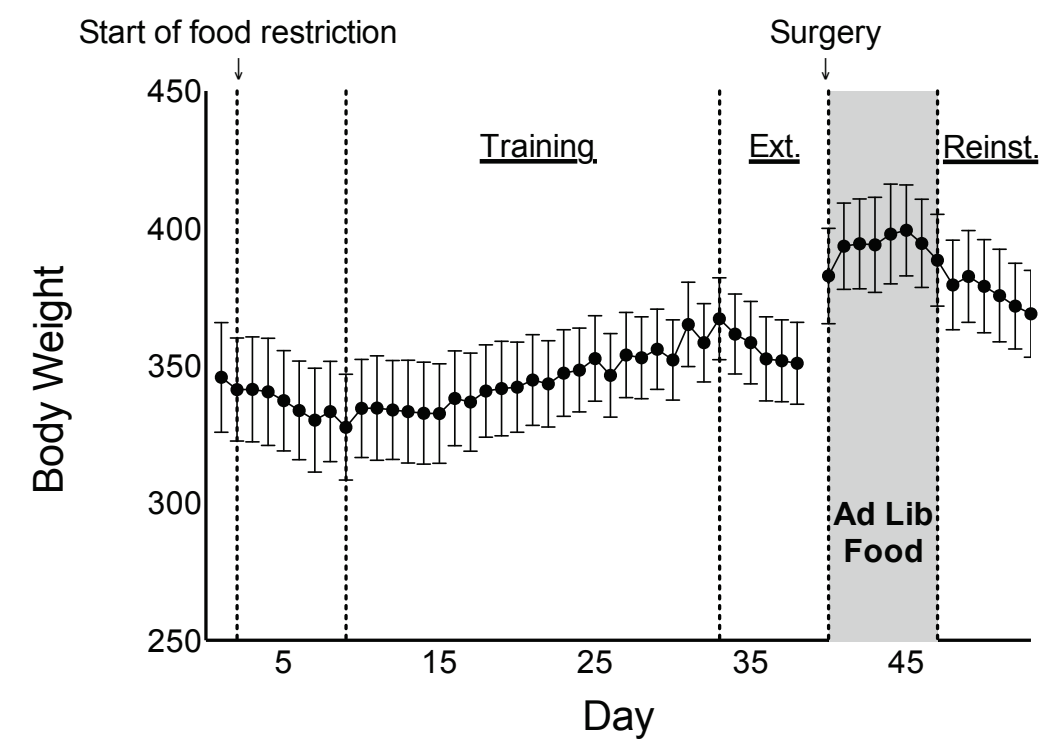

Figure 18. Mean (+/- SEM) body weight in grams of LE rats throughout Experiment $4 A(n=18$ rats $)$. 
Training and Extinction: The number of pellets earned and the number of active lever time out responses increased significantly over the training phase $(\mathrm{p}<0.001$ in both cases) (Figure 19A). As previously reported (Ghitza et al., 2007, 2006; Nair, AdamsDeutsch, Pickens, et al., 2009; Nair et al., 2008), the progressive escalation of time out responses was greater than that of pellets earned (i.e., active lever responding that did not occur during time out periods), such that there were over 4 times as many time out responses than rewarded ones on the last day of training $(p<0.001)$. The number of inactive lever presses did not increase over time $(p=0.627)$. During the extinction phase, the analyses revealed significant decreases in both active lever pressing and inactive lever pressing over time $(\mathrm{p}<0.001)$ (Figure 19B). 
A

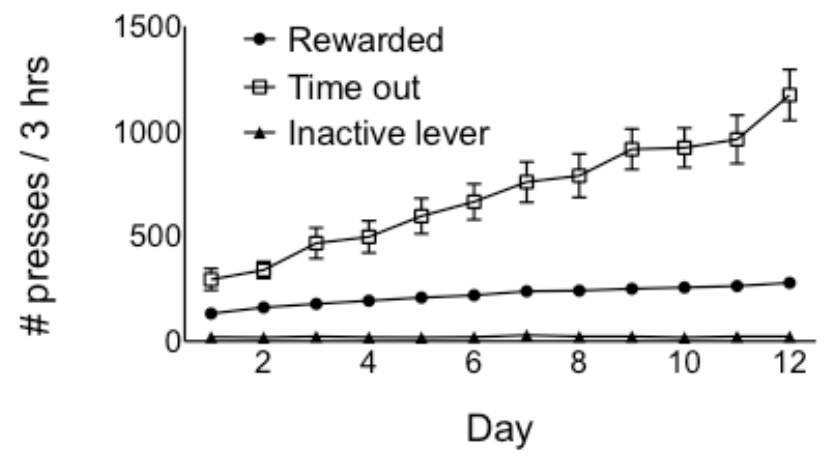

B

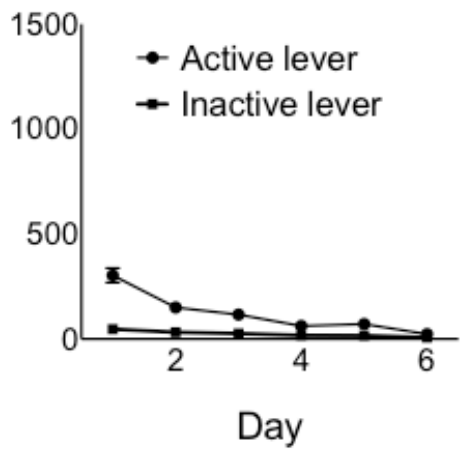

Figure 19. Experiment 4A: Mean (+/- SEM) number of pellets earned, time out responses, and inactive lever presses during training (A) and mean (+/- SEM) number of active and inactive lever presses during extinction (B). 
Post-surgery analyses: A total of 7 rats were excluded from the remaining analyses due to post-surgical complications. Histological examination revealed inaccurate cannula placements in 10 of the remaining rats. One rat was excluded for failing to reach the extinction criterion. The final numbers of rats in the saline, ghrelin, and [D-Lys-3]GHRP-6 groups were 5, 6 and 7, respectively. Cannula placements are shown in Figure 20. A cresyl violet stain revealed normal cell morphology around the cannula tract, indicating minimal histological damage (Figure 21). 

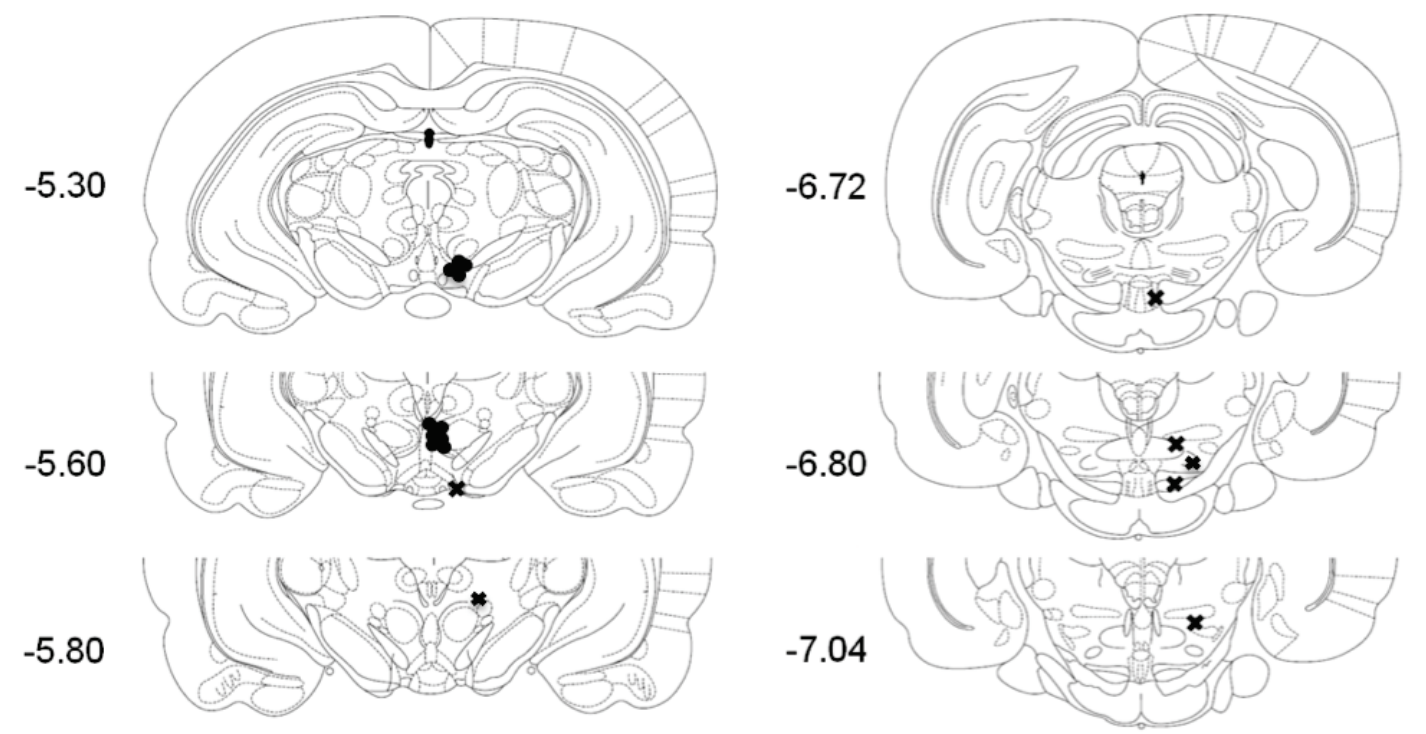

$-6.04$
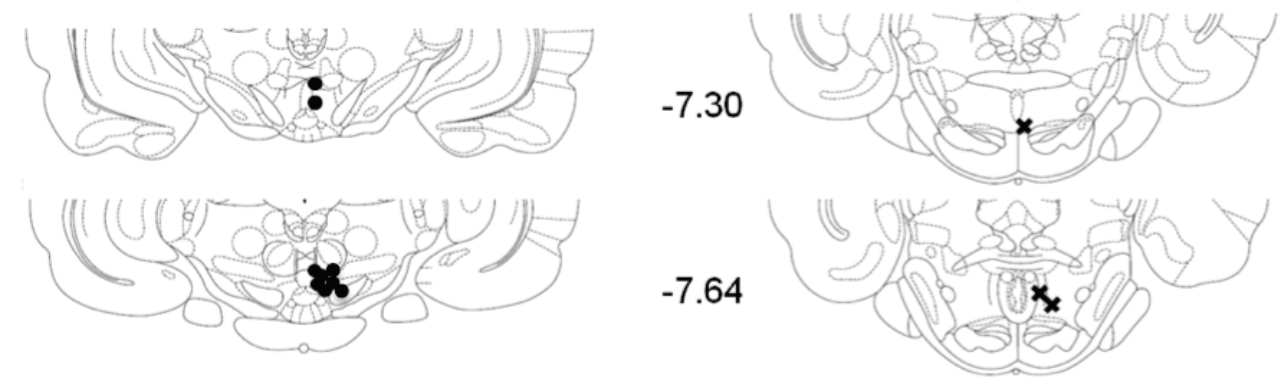

$-6.30$

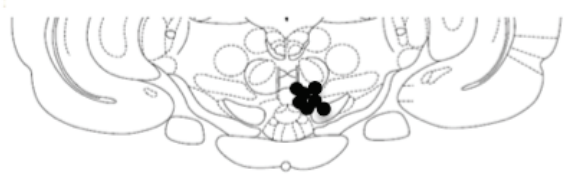

$-7.64$

Figure 20. Accurate $(\bullet)$ and inaccurate $(\boldsymbol{x})$ cannula placements for each level on the rostro-caudal axis (Paxinos and Watson, 1998) in Experiment 4A. 

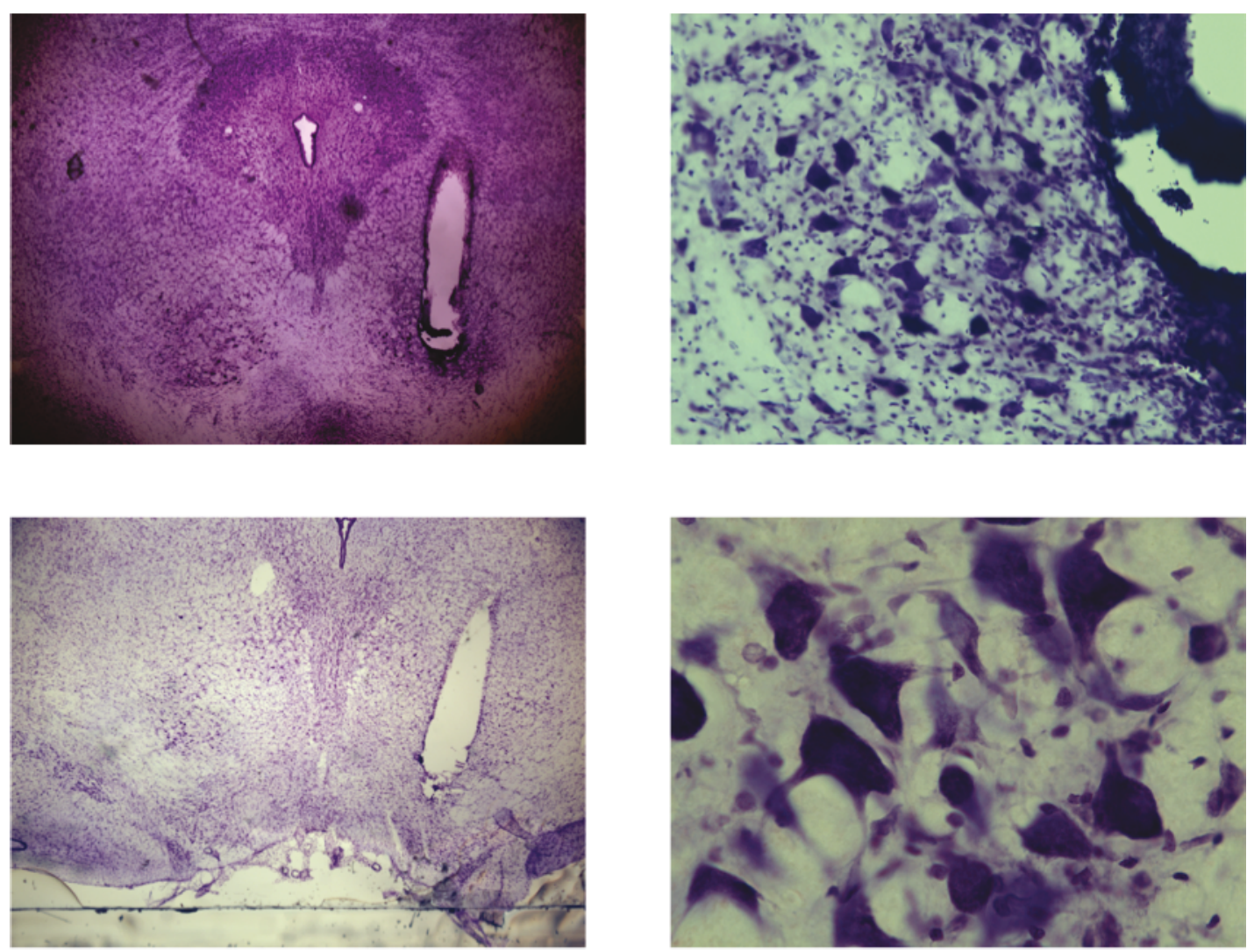

Figure 21. Experiment 4A: Top and bottom left: representative photograph of cannula placements in the ventral tegmental area (VTA) at 2.5 magnification. Cells around the cannula tract at 10 magnification (top right) and 60 magnification (bottom right) 
Post-surgery food intake: Chow intake following surgery did not differ between drug treatment groups $[\mathrm{F}(2,14)=0.964, \mathrm{p}=0.405$; partial eta squared $=0.121]$ (Figure 22A). [D-Lys-3]-GHRP-6-treated rats gained less weight during this time than saline- and ghrelin-treated rats $([\mathrm{F}(2,17)=6.702, \mathrm{p}=0.008$, partial eta squared $=0.472]$; LSD post hoc values: $\mathrm{p}<0.01$ in both cases) (Figure 22B). Importantly, absolute body weights went up after the surgery $[\mathrm{t}(17)=-2.397, \mathrm{p}=0.028, \mathrm{~d}=-1.102]$, indicating a healthy recovery in all animals. Table 4 displays each group's relative weights (as a percent of baseline weight) at the beginning and end of the reinstatement phase. 


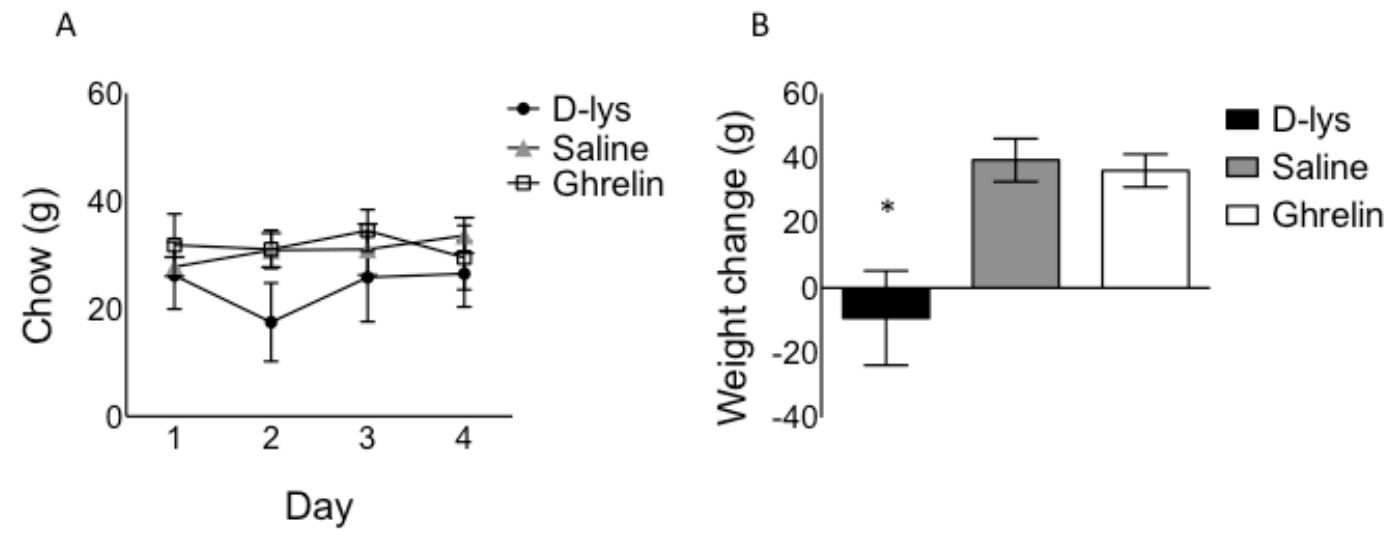

Figure 22. Experiment 4A: Mean (+/- SEM) chow intake (A) and weight gain (B) following surgery. $(*)$ denotes $\mathrm{p}<0.01$. 
Table 4. Relative body weights (in percentage of baseline) at the start and end of reinstatement period (Experiment 4A)

\begin{tabular}{|c|c|c|c|c|c|c|}
\hline & \multicolumn{2}{|c|}{ Saline $(n=5)$} & \multicolumn{2}{|c|}{ Ghrelin $(n=6)$} & \multicolumn{2}{|c|}{ D-Lys $(n=7)$} \\
\hline Time point & $\begin{array}{c}\text { Mean } \\
\pm \text { SEM }\end{array}$ & $\mathbf{p}$ & $\begin{array}{c}\text { Mean } \\
\pm \text { SEM }\end{array}$ & $\mathbf{p}$ & $\begin{array}{c}\text { Mean } \\
\pm \text { SEM }\end{array}$ & $\mathbf{p}$ \\
\hline Baseline & $\begin{array}{l}100 \\
\pm 0\end{array}$ & $\mathrm{n} / \mathrm{a}$ & $\begin{array}{l}100 \\
\pm 0\end{array}$ & $\mathrm{n} / \mathrm{a}$ & $\begin{array}{l}100 \\
\pm 0\end{array}$ & $\mathrm{n} / \mathrm{a}$ \\
\hline First reinstatement day & $\begin{array}{r}119.59 \\
\pm 2.99 \\
\end{array}$ & $0.003 *$ & $\begin{array}{r}116.03 \\
\pm 5.78 \\
\end{array}$ & $0.039 *$ & $\begin{array}{l}108.38 \\
\pm 4.55 \\
\end{array}$ & 0.115 \\
\hline Last reinstatement day & $\begin{array}{r}115.07 \\
\pm 3.02 \\
\end{array}$ & $0.008 *$ & $\begin{array}{l}109.73 \\
\pm 4.93\end{array}$ & 0.105 & $\begin{array}{l}102.29 \\
\pm 4.30 \\
\end{array}$ & 0.614 \\
\hline
\end{tabular}


Reinstatement: A repeated measure ANOVA for active lever pressing with reinstatement stimulus as the within subject factor and drug treatment condition as the between subject factor revealed a significant interaction effect $[\mathrm{F}(6,45)=3.026, \mathrm{p}=$ 0.014, partial eta squared $=0.474]$. A series of ANOVAs revealed that drug treatment did not affect active lever pressing in the food priming- and fasting-induced reinstatement conditions, and during the extinction session (no reinstatement stimulus), as indexed by comparable responding across treatment groups (food priming: $[\mathrm{F}(2,17)=0.908, \mathrm{p}=$ 0.424]; fasting: $[\mathrm{F}(2,17)=1.069, \mathrm{p}=0.368]$; extinction: $[\mathrm{F}(2,17)=0.120, \mathrm{p}=0.887]) . \mathrm{A}$ significant effect of drug treatment on active lever pressing was found in the cue-induced reinstatement condition $[\mathrm{F}(2,17)=5.625, \mathrm{p}=0.015$, partial eta squared $=0.429]$. A follow up LSD test revealed that under this condition, ghrelin-treated rats had greater responding than [D-Lys-3]-GHRP-6-treated rats $(\mathrm{p}=0.005)$ and saline-treated rats, although this was only a marginal effect $(\mathrm{p}=0.067)$ (Figure 23). Inactive lever pressing and locomotor activity did not differ between the treatment groups in any of the reinstatement conditions and during extinction $(\mathrm{p}>0.05)$ (data not shown). 


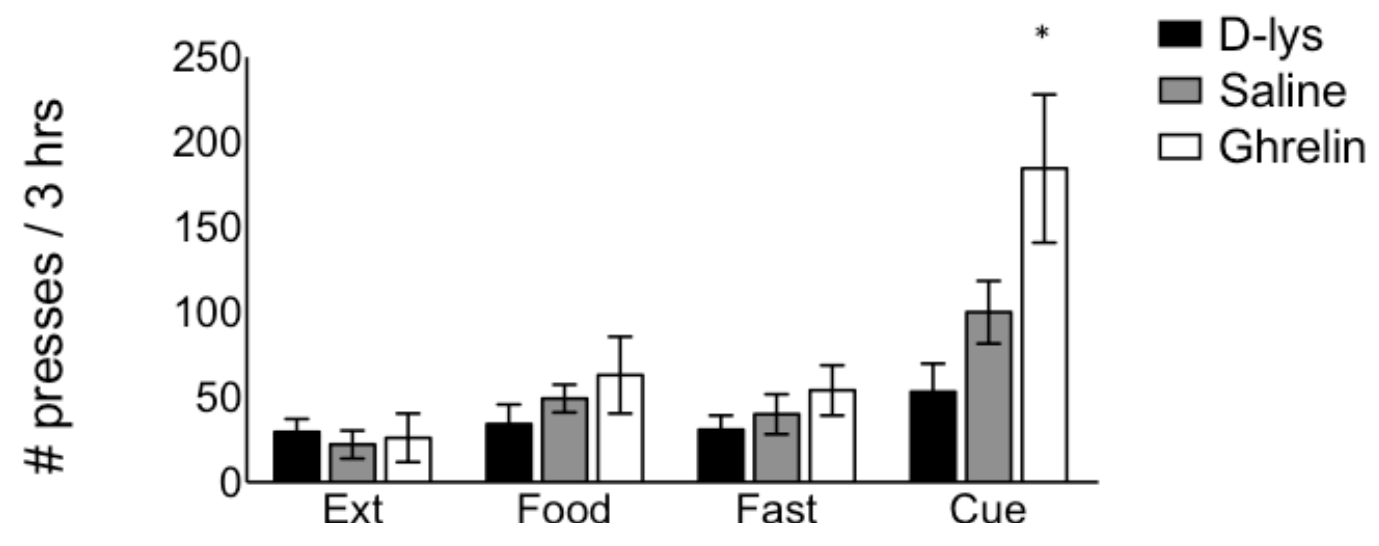

Figure 23. Experiment 4A: Mean (+/- SEM) number of active lever presses during food-priming-, fast-, and cue-induced reinstatement and during an extinction session (no reinstatement stimulus). (*) denotes higher responding in ghrelin-treated rats versus d-lys-treated rats (significant, $\mathrm{p}=0.005$ ) and saline-treated rats (approaching significance, $\mathrm{p}=0.067$ ) in the cue-induced reinstatement condition. 
Finally, a series of paired t tests were conducted to assess the efficacy of the reinstatement stimuli in saline-treated rats (i.e. difference between each reinstatement condition and the extinction session in GHSR-manipulation free rats). The analyses revealed that in saline-treated (control) rats, food associated cues were effective in reinstating the active lever pressing response (as indicated by higher responding in this condition than during extinction), but this effect did not reach significance for the food priming condition (cue: $\mathrm{p}<0.012, \mathrm{~d}=-2.436$; food priming: $\mathrm{p}=0.101$ ). As expected, similar analyses revealed that ghrelin-treated rats had higher active lever responding in the cue-induced reinstatement session relative to extinction, but this effect did not reach statistical significance in food priming-induced reinstatement (cue: $p=0.029, d=-3.429$; food priming: $p=0.073$ ). An overnight fast did not induce reinstatement in either group (saline: $p=0.290$, ghrelin: $0=0.249$ ). Finally, [D-Lys-3]-GHRP-6-treated rats had comparable active lever responding in all reinstatement sessions relative to the extinction session (cue: $\mathrm{p}=0.199$; food priming: $\mathrm{p}=0.662$; fasting: $\mathrm{p}=0.851$ ).

\section{Experiment 4B:}

Pre and post surgery weights: Rats' weights over the course of the experiment fluctuated as a function of feeding regimen (food restricted vs. adlib). A series of paired t tests revealed that rats weights decreased over the course of initial food restriction $[\mathrm{t}(15)$ $=2.465, \mathrm{p}=0.026]$, training $[\mathrm{t}(15)=2.110, \mathrm{p}=0.052]$, and extinction $[\mathrm{t}(15)=3.818, \mathrm{p}=$ 0.003]. Weight loss throughout training and extinction was also observed when only examining the 13 rats tested for reinstatement (see below; $p<0.05$ ). When returned on an adlib feeding schedule, rats tested for reinstatement gained weight between the end of the extinction phase and the day of the surgery $[\mathrm{t}(12)=-5.748, \mathrm{p}=0.000]$ and during the days 
following surgery $[t(12)=-2.273, p=0.042]$. When food restricted for a second time, they lost weight prior to $[\mathrm{t}(12)=7.185, \mathrm{p}=0.000]$ and during the reinstatement phase $[\mathrm{t}(12)=2.517, \mathrm{p}=0.027]$ (Figure 24). In these rats, body weights were similar at the start and end of the reinstatement phase as they had been at baseline ( $p>0.05$ in both cases). 


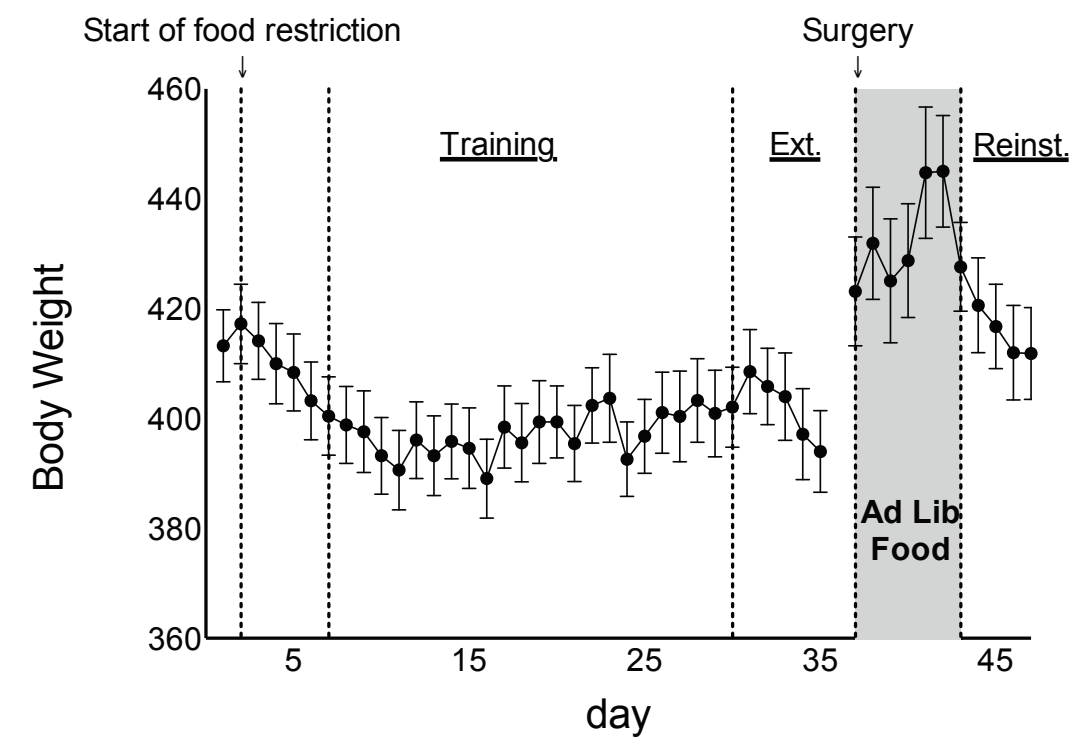

Figure 24. Mean (+/- SEM) body weight in grams of LE rats throughout experiment $4 B(n=13$ rats $)$. 
Training and Extinction: Rats' operant responding during training and extinction was similar as in Experiment 4A. The number of pellets earned and the number of active lever time out responses increased significantly over the course of training $(p<0.001$ in both cases). The progressive escalation of time out responses was greater than that of pellets earned, such that there were over 5 times as many time out responses as rewarded ones on the last day of training $(\mathrm{p}<0.001)$ (Figure 25A). The number of inactive lever presses did not change over time $(\mathrm{p}=0.559)$. During the extinction phase, the analyses revealed significant decreases in both active and inactive lever presses over time (active lever: $\mathrm{p}<0.001$; inactive lever: $\mathrm{p}=0.045$ ) (Figure 25B). 

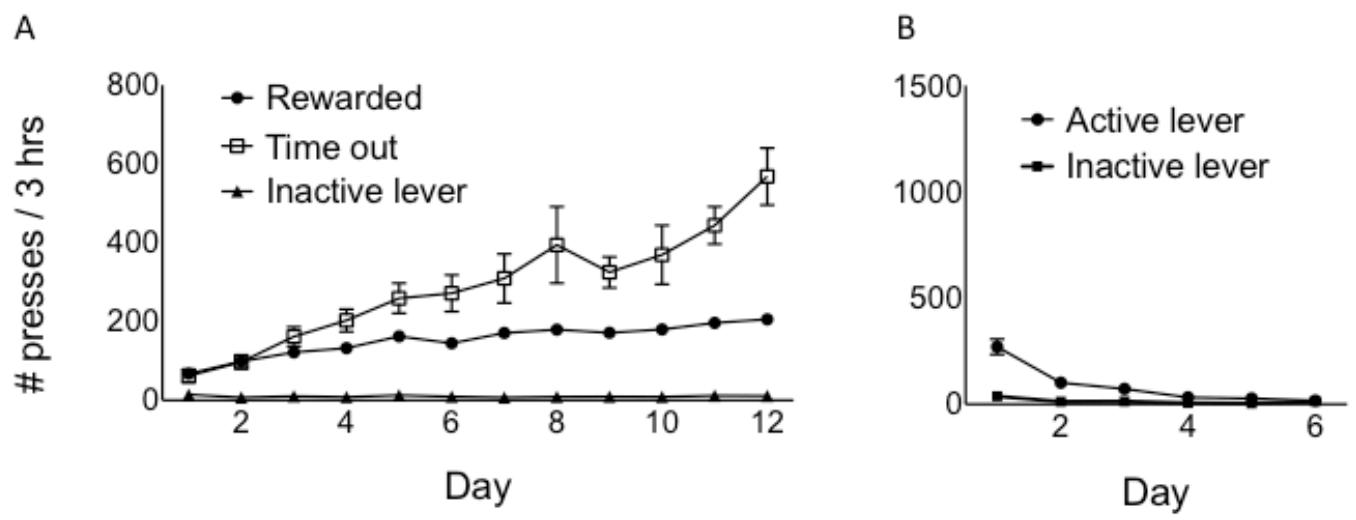

Figure 25. Experiment 4B: Mean ( $+/-$ SEM) number of pellets earned, time out responses, and inactive lever presses during training (A) and mean (+/- SEM) number of active and inactive lever presses during extinction (B). 
Post-surgery analyses: Two rats were excluded from the remaining analyses due to post-surgical complications. Histological examination revealed an inaccurate cannula placement in 1 additional rat. The final numbers of rats in the saline and [D-Lys-3]GHRP-6 groups were 7 and 6, respectively. Cannula placements are shown in Figure 26. 

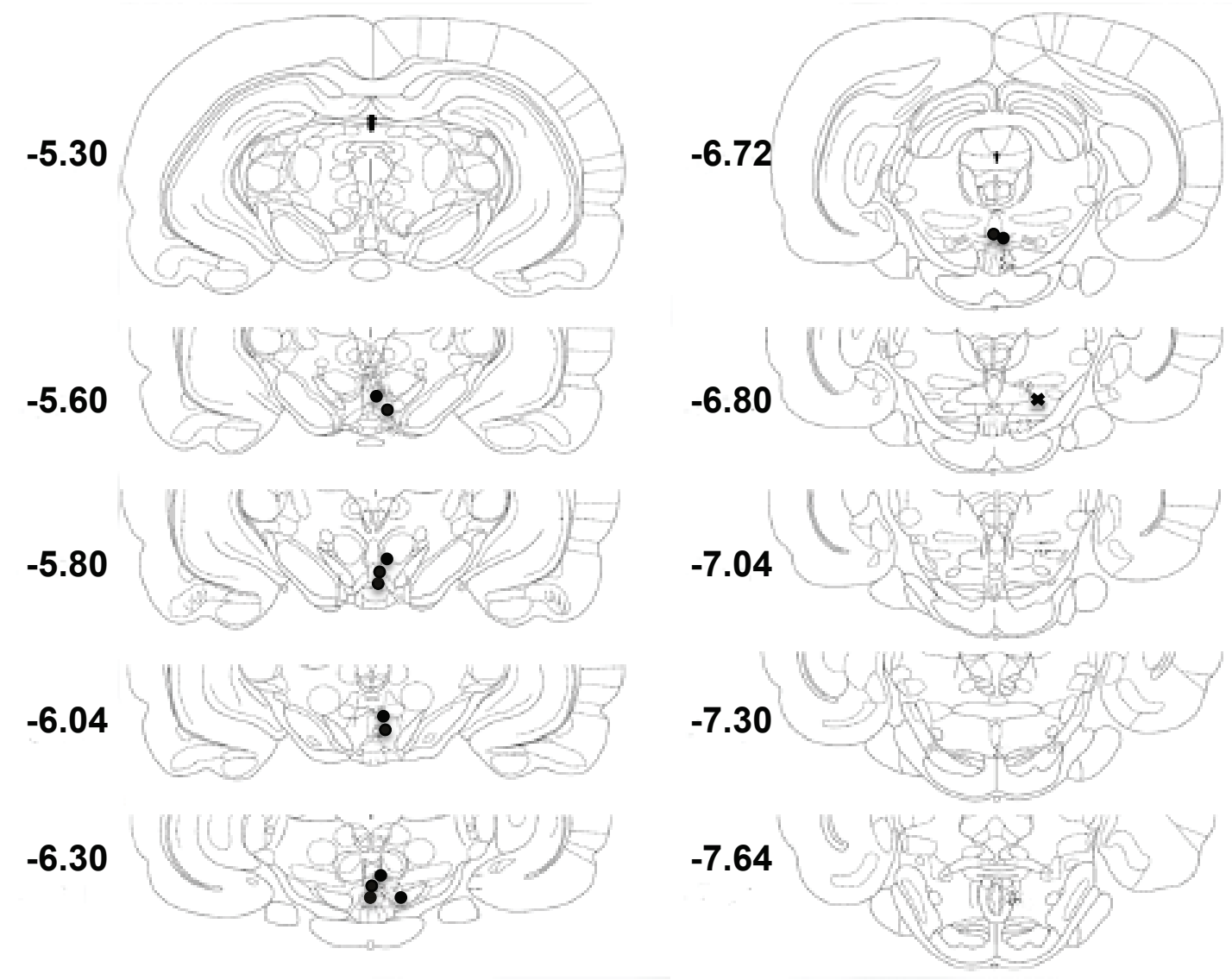

Figure 26. Accurate $(\bullet)$ and inaccurate $(\mathbf{x})$ cannula placements for each level on the rostro-caudal axis (Paxinos and Watson, 1998) in Experiment 4B. 
Post-surgery food intake: A repeated measure ANOVA revealed that [D-Lys-3]GHRP-6 rats ate significantly less than saline-treated rats in the days following surgery $([\mathrm{F}(1,11)=11.456, \mathrm{p}=0.006]$; there was no main effect of day or day by treatment group interactions, $p>0.05$ ) (Figure 27A). A second repeated measure ANOVA with postsurgery day as a within subjects factor and drug treatment group as a between subjects factor revealed a significant main effect of day $([\mathrm{F}(4,44)=4.010, \mathrm{p}=0.007]$; rats gained weight over time), a significant day by drug treatment group interaction $[F(4,44)=6.024$, $\mathrm{p}=0.001]$, but no main effect of drug treatment group $[\mathrm{F}(1,11)=1.660, \mathrm{p}=0.224]$. A series of paired $t$ tests comparing each day to the next within each individual drug treatment group was used to follow up on the interaction effect. The tests revealed that while saline-treated rats gained weight over time (significant differences between days 0 (i.e. surgery day) and 1, 1 and 2, and 3 and 4, p $<0.05$ ), [D-Lys-3]-GHRP-6-treated rats did not (all p values $>0.05$ ) (Figure 27B). 

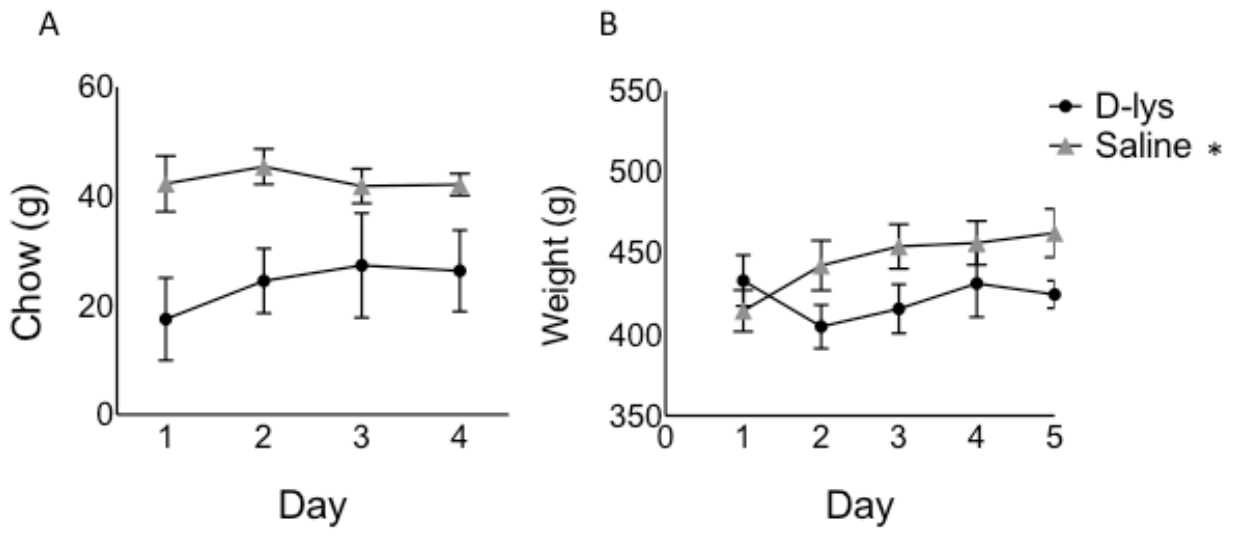

Figure 27. Experiment 4B. Mean (+/- SEM) chow intake (A) and weight gain (B) following surgery. 
Reinstatement: A repeated measure ANOVA for active lever pressing with reinstatement condition (yohimbine vs. vehicle) as the within subject and drug treatment condition as the between subjects factor revealed a significant main effect of reinstatement condition $[F(1,11)=6.068, p=0.031]$, but no drug treatment effect and no reinstatement condition by drug treatment interaction (both $p$ values $>0.05$ ). All rats displayed higher active lever responding after a yohimbine injection than after a vehicle injection (Figure 28). It is noteworthy that the observed power of the drug treatment condition by reinstatement condition interaction was only 0.246 . Inactive lever pressing and locomotor activity did not differ between the drug treatment conditions in either of the reinstatement conditions $(\mathrm{p}>0.05)$ (data not shown). 


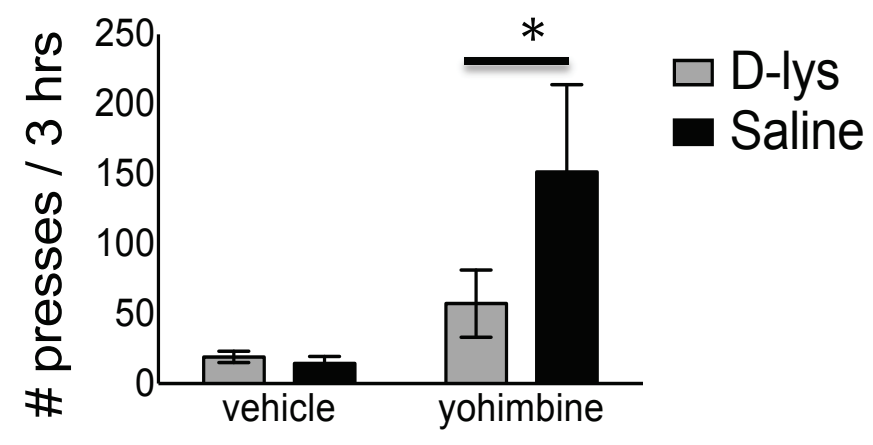

Figure 28. Experiment 4B. Mean (+/- SEM) number of active lever presses during yohimbine-induced reinstatement and a control session with vehicle only. ${ }^{*}$ denotes a main effect of reinstatement condition $(\mathrm{p}<0.05)$. 


\section{Discussion}

Experiment 4A: Cue-induced reinstatement of high fat food seeking

The main finding of experiment $4 \mathrm{~A}$ is that intra-VTA ghrelin administration enhances cue-induced reinstatement of high fat food seeking in food-restricted rats. In addition, cues failed to elicit reinstatement relative to an extinction session in [D-Lys-3]GHRP-6 treated rats, but not in ghrelin-treated rats or saline-treated controls. This suggests that ghrelin signalling in the VTA is necessary for the expression of cue-induced reinstatement of high fat food seeking.

It is unlikely that these findings resulted from group differences in hunger or unspecific arousal, since similar effects were not seen following other types of reinstatement stimuli or during an extinction session. In addition, drug treatment condition did not affect inactive lever responding or locomotion scores in any of the sessions. Thus, it appears that ghrelin specifically potentiated the response to cues associated with the high-fat food reward during training.

These results are consistent with those of Tessari et al. (2007), who showed a significant positive correlation between endogenous ghrelin levels and reinstatement of cocaine seeking following exposure to a cocaine-associated cue. Importantly, the implication that ghrelin signalling can potentiate the behavioural response to cues is in line with recent findings that ghrelin infusions increase the number of meals eaten by sated rats in response to conditioned stimuli (Kanoski et al., 2013). In addition, systemic blockade of the GHSR attenuates cue-potentiated feeding (Walker et al., 2012). It should be noted, however, that in a Pavlovian-to-Instrumental (PIT) model, systemic [D-Lys-3]GHRP-6 administration was found to enhance food consumption in response to 
conditioned stimuli (Johnson, Canter, Gallagher, \& Holland, 2009). This could be due to region-specific effects of [D-Lys-3]-GHRP-6, as dissociations in PIT performance and sucrose ingestion have previously been reported in lesions studies (Holland \& Gallagher, 2003).

Theoretically, ghrelin's effects on cue-potentiated feeding/food seeking in this study and others could be due to an enhanced association between the food and conditioned stimuli and/or memory retention. Several studies show that ghrelin facilitates memory acquisition and recall (Albarran-Zeckler, Brantley, \& Smith, 2012; Carlini et al., 2004; Davis et al., 2011; Diano et al., 2006) and promotes dendritic spine formation and long-term potentiation in the hippocampus (Diano et al., 2006). However, group differences in learning/memory are unlikely to account for the results of the current study as ghrelin and [D-Lys-3]-GHRP-6 treatments did not start until after training and extinction. Instead, it appears that intra-VTA ghrelin administration enhanced the incentive value of food cues and the motivation to act upon them, which in turn triggered reinstatement. It is likely that ghrelin's effects occurred via the activation of local DA cells that project to the nucleus accumbens (NAc). As previously mentioned, ghrelin binding in the VTA lowers the firing threshold of these neurons and increases DA turnover in the NAc (Abizaid et al., 2006). VTA DA cells respond to cues associated with food rewards (Mirenowicz \& Schultz, 1996; Schultz, Apicella, \& Ljungberg, 1993), and VTA-NAc connections have previously been implicated in sucrose seeking in response to conditioned stimuli (Yun, Wakabayashi, Fields, \& Nicola, 2004). The activation of VTA DA cells may be a direct result of ghrelin's action on these cells, or may be mediated by local GABAergic cells. Approximately $30 \%$ of VTA GABAergic cells express the GHSR 
(Abizaid et al., 2006), and activation of these cells has been observed during states of reward expectancy and reward seeking behaviours (Steffensen, Lee, Stobbs, \& Henriksen, 2001).

Previous studies suggest different mechanisms underlie stress-, priming-, and cueinduced reinstatement of food seeking (reviewed in Nair, Adams-Deutsch, Epstein, et al., 2009). Such differences could account for the fact that ghrelin's effects on cue-induced reinstatement did not generalize to food priming or an overnight fast. Further, available data suggest that food priming-induced food seeking reinstatement is particularly sensitive to feeding regimen, and that the effects of this manipulation are weaker in adlib rats than food restricted rats (Nair, Adams-Deutsch, Epstein, et al., 2009). In contrast with previous studies (in which rats undergoing this test were food restricted for the entire duration of the experiment, Ghitza et al., 2007, 2006; Nair, Adams-Deutsch, Pickens, et al., 2009), rats in experiment 4A had unlimited access to chow for at least 5 days prior to reinstatement. As a result, rats were heavier at the beginning and end of the reinstatement phase than at baseline, and likely under a lesser metabolic challenge at the time of testing than rats in previous studies. The fact that food priming did not significantly increase lever pressing relative to extinction in control rats in the current study provides additional support that metabolic status is a key determinant in the expression of this type of reinstatement. In this respect, it is interesting that ghrelintreated rats showed greater food seeking responses following food priming than during an extinction session. Although this effect did not reach statistical significance, it raises the possibility that manipulations that increase an animal's motivation to seek palatable rewards (King et al., 2011) may also decrease the impact of metabolic status in the 
expression of food priming-induced reinstatement; this however, remains to be tested directly.

Finally, an overnight fast failed to elicit reinstatement in any of our groups, including the control group. It should be noted, however, that in a previous study, [DLys-3]-GHRP-6 did not alter fasting-induced reinstatement of heroin seeking (Maric et al., 2012). Importantly, the effects of this manipulation have, to our knowledge, primarily been investigated in drug seeking reinstatement studies (e.g. Maric et al., 2012; Shalev, Marinelli, Baumann, Piazza, \& Shaham, 2003; Shalev, Yap, \& Shaham, 2001). It is therefore possible that food deprivation is simply not an effective reinstatement stimulus for food seeking. A prolonged food restriction paradigm may be more likely to produce a motivational state that allows for an overnight fast to produce reinstatement responses (Carr, 2007). Furthermore, a number of mild stressors known to induce reinstatement of drug seeking in the operant conditioning model do not reliably induce reinstatement of food seeking (Nair, Adams-Deutsch, Epstein, et al., 2009).

Experiment 4B: Yohimbine-induced reinstatement of sucrose pellets

Yohimbine is considered a more reliable reinstatement stimulus than a variety of other stressors (reviewed in Nair, Adams-Deutsch, Epstein, et al., 2009; See \& Waters, 2011). It has previously been suggested that this is because stronger stressors induce a flight or fight response that is incompatible with food seeking, and that at doses similar to that used in the current study, yohimbine's effects on appetitive behaviours are stronger than its effects on anorectic stress systems (Nair, Adams-Deutsch, Epstein, et al., 2009). Accordingly, yohimbine was effective in reinstating sucrose pellet seeking in LE rats in experiment 4B. The data do not support a role of ghrelin in this phenomenon. 
Nevertheless, it is important to note that the power to detect such an effect in the analyses was very low, possibly because of the small sample sizes. We recommend that future experiments examining the effects of ghrelin signalling in yohimbine-induced reinstatement of food seeking have larger samples.

Intra-VTA GHSR signalling and food intake

Several studies report changes in food intake following VTA GHSR activation or antagonism (Abizaid et al., 2006; King et al., 2011; Naleid et al., 2005; Skibicka et al., 2011). In the current study, intra-VTA ghrelin did not affect chow intake in the week following surgery, and intra-VTA [D-Lys-3]-GHRP-6 administration only significantly reduced chow intake in experiment 4B. Discrepancies between our findings and others' may be explained by differences in the administration protocol. Most studies examined the effects of acute ghrelin or GHSR antagonist infusions in awake animals, and observed effects lasting from 1 to 3 hours (Abizaid et al., 2006; Naleid et al., 2005; Skibicka et al., 2011). Only one study examined the effects of chronic intra-VTA ghrelin/[D-Lys-3]GHRP-6 treatment (King et al., 2011). The changes in food intake observed in that study took place over the entire infusion period, which lasted 14 days. Thus, it is conceivable that the window during which food intake was measured in the current study (4 days within the first week following surgery) did not allow for the detection of acute (immediately after surgery) or chronic effects of VTA GHSR manipulations on feeding. It is also possible that the rats' long history of food restriction (during the training and extinction phases, i.e. over 1 month) caused them to overeat during the week following surgery, thus making ghrelin and/or [D-Lys-3]-GHRP-6 effects difficult to detect (ceiling effect). Finally, it should be noted that in a free choice experiment, King et al. (2011) 
found that chronic intra-VTA [D-Lys-3]-GHRP-6 administration decreased consumption of a high fat diet, but had no effect on less preferred protein-rich and carbohydrates-rich diets. In addition, upon ghrelin administration, VTA-lesioned rats ate less peanut butter but similar amounts of regular chow compared to sham-lesioned rats (Egecioglu et al., 2010). Thus it is possible that ghrelin's orexigenic effects in the VTA are particularly relevant to palatable rewarding foods.

The fact that ghrelin affected cue-induced reinstatement of food seeking but not post-surgery food intake is in line with reports that intra-VTA ghrelin administration at doses that are insufficient to promote food intake increases operant responding for sucrose (Skibicka et al., 2011). This provides additional support that reward behaviors are more sensitive to intra-VTA ghrelin than feeding responses.

\section{Operant conditioning measures}

Response rates during training and extinction were consistent with a number of previous studies using similar rewards and reinforcement schedules (Ghitza et al., 2007, 2006; Nair, Adams-Deutsch, Pickens, et al., 2009; Nair et al., 2008). Notably, whereas the number of high fat chocolate pellets earned reached a plateau early on during training (around days 5-6), the number of non-reinforced active lever presses progressively increased until the end of the training phase. By the end of this phase, rats acquired an average of 282 pellets and performed an average of 1240 non-reinforced responses over the course of three hours. Ghitza and colleagues (2006) reported a similar pattern and suggested that this reflects the development of compulsive food seeking. Rats took an average of 7 days to reach the extinction criterion, defined as less than 30 active lever presses per 3-hour session on 3 consecutive days, and only one rat (experiment 4A) 
reached the maximum number of extinction sessions. Inactive lever pressing responses remained consistently low throughout training and extinction of both experiments.

\section{$\underline{\text { Limitations and conclusions }}$}

One potential caveat of the current study is that [D-Lys-3]-GHRP-6 has previously been shown to activate 5HT-2B receptors (Depoortere, Thijs, \& Peeters, 2006), raising the possibility that its action in the current study was not mediated by the GHSR. However, we believe that this is unlikely, given that ghrelin-treated rats exhibited greater cue-induced reinstatement relative to controls as well as [D-Lys-3]-GHRP-6 treated rats. Further, food reward studies using this GHSR antagonist and more selective ones typically yield similar results (King et al., 2011; Landgren, Simms, et al., 2011; Skibicka et al., 2011; Skibicka, Hansson, et al., 2012).

Another potential caveat of the current study is the possibility that the relatively large volume of the solutions infused may have caused leakage into surrounding tissues. However, previous studies have shown that ghrelin's acute orexigenic effects in the VTA are not present in rats when the cannula is placed in areas adjacent to the VTA (Abizaid et al., 2006). Similarly, ghrelin does not increase locomotion or accumbal DA levels when injected in close proximity to, but not directly in the VTA (Jerlhag et al., 2007). This indicates limited effects of ghrelin in areas surrounding the VTA and any effect of ghrelin or [D-Lys-3]-GHRP-6 following minipump infusion is therefore most likely attributable to their effects directly in the VTA. 


\section{Conclusion}

In conclusion, the findings in experiment 4 indicate that ghrelin facilitates the reinstatement of food seeking in response to cues, and that this effect can happen at the level of the VTA. To the extent that the reinstatement paradigm is considered a valid model of relapse in humans (Epstein et al., 2006; Nair, Adams-Deutsch, Epstein, et al., 2009), this suggests that ghrelin may, under some circumstances, trigger relapse to preferred foods in dieting individuals. Although the precise mechanisms underlying this effect are currently unknown, the findings of the present investigation fit in well with current theories that diet-induced ghrelin elevations may contribute to weight regain after the diet (Cummings et al., 2002). 


\section{Chapter 7: General discussion}

The overarching goal of the present thesis was to extend our current knowledge regarding the role of ghrelin in reward-based feeding. Specifically, the experiments described in chapters 3 to 6 were designed to test the hypotheses that ghrelin signalling contributes to dessert consumption in sated animals as well as relapse to food seeking following a period of (forced) abstinence. In addition, the experiments described in chapters 3 to 5 aimed to further characterize a relatively new genetic model of GHSR function, the GHSR-KO rat.

In Chapter 3, cookie dough consumption after a meal was studied in GHSR-KO and WT rats trained to consume the majority of their daily food intake within a set period during the day. This paradigm was designed to model dessert consumption in humans $-\mathrm{a}$ form of reward-based overeating that may result in obesity. Relative to WT rats, GHSRKO rats consumed significantly less of the cookie dough treat when sated. Although this finding was not paralleled by obvious differences in the activation of the reward system, a follow up experiment demonstrated that ghrelin signalling does affect the neuronal response to food cues that predict imminent access to a treat in the core of the nucleus accumbens. This region is sensitive to conditioned stimuli (CS) associated with rewards and is thought to support CS' ability to drive goal directed behaviours.

Using the same genetic model, experiments in chapters 4 and 5 did not support a role for ghrelin signalling in operant responding for sucrose and high fat chocolateflavoured pellets, reinstatement of food seeking after a period of extinction, or the development of a place preference for cookie dough. This may of course indicate that ghrelin is not involved in these behaviours. This however seems unlikely given that 
previous studies demonstrate that ghrelin administration increases (and GHSR antagonists decrease) operant responding for rewarding substances including palatable foods (heroin, alcohol, sucrose solution, as well as banana-flavored, sugar and high fat pellets) (Kanoski et al., 2013; King et al., 2011; Landgren et al., 2012; Landgren, Simms, et al., 2011; Maric et al., 2012; Overduin et al., 2012; Perello et al., 2010; Skibicka, Hansson, et al., 2012; Skibicka, Shirazi, et al., 2012; Skibicka et al., 2013, 2011; Weinberg et al., 2011) and facilitates CPP to cocaine, nicotine, chocolate, and a high fat diet (Davis et al., 2007; Egecioglu et al., 2010; Jerlhag \& Engel, 2011; Perello et al., 2010), and that other genetic models of ghrelin signalling disruptions are resistant to these effects (Bahi et al., 2013; Davis et al., 2012; Jerlhag et al., 2009, 2010). Alternatively, it may be the case that the present GHSR-KO rat model is better suited for some tasks than others. So far, available data concerning these animals indicate that they can be used to look at ghrelin's role in intra-cranial self-stimulation (ICSS) and locomotor sensitization paradigms (Clifford et al., 2012; Wellman et al., 2012) as well as dessert consumption and food anticipatory activity (FAA) (current thesis). However, given that both GHSR-KO and WT rats were much less responsive to a continuous schedule of reinforcement in an operant task than what is typically seen when using LE rats under the same conditions, it is possible that FHH rats are generally less willing to perform work for (or perform an approach response to) food rewards than other rat strains. If this is the case, then it may be harder to detect any effect of ghrelin signalling in the operant conditioning and CPP tests using this model (floor effects). The precise nature of the FHH rats' deficits in the operant conditioning task is currently unclear. As mentioned, it is possible that relative to LE rats, FHH rats are less motivated to obtain 
food rewards. Alternatively, FHH rats may exhibit impaired food reward learning. In either scenario, it is significant that the present thesis established an effect of the GHSR KO genotype in dessert consumption and FAA in spite of the limitations inherent to the model. This further strengthens the association between ghrelin signalling and these behaviours.

Notably, in every experiment where effects of ghrelin signalling on reward behaviours were observed by comparing GHSR-KO and WT rats, the phenotype of interest was not abolished in GHSR-KO rats, but simply attenuated. Sub-threshold doses of cocaine eventually increase locomotion in GHSR-KO rats (locomotor sensitization), but this effect is not as pronounced as in WT rats (Clifford et al., 2012). Similarly, GHSR-KO rats exhibit the same type of responding to ICSS as WT rats, but require higher stimulation intensities (Wellman et al., 2012). In the present thesis, GHSR-KO rats did consume dessert and show FAA, but this effect was attenuated compared to WT rats. These findings may indicate that ghrelin is not the sole mediator of reward behaviours. They may also suggest that rats developing in the absence of the GHSR gene acquire compensatory mechanisms that limit the effects of the genetic modification. Indeed, such compensatory mechanisms are thought to exist in several genetic model of ghrelin signalling disruptions, although they are less obvious in animals lacking the genes for both ghrelin and the GHSR (Davis et al., 2012; Egecioglu et al., 2010; Jerlhag, Egecioglu, et al., 2011; Pfluger et al., 2008).

Given the limitations of the GHSR-KO model in the operant conditioning paradigm, the experiments described in Chapter 6 used a pharmacological manipulation in outbred rats to examine ghrelin's role in the reinstatement of palatable food seeking 
triggered by food cues, a food prime, an overnight fast and the pharmacological stressor yohimbine. These experiments also tested the hypothesis that ghrelin contributed to this reinstatement by acting at the level of the VTA. Only cues and yohimbine were effective in triggering reinstatement in control rats. As mentioned in chapter 6, this may be related to the metabolic state of the animals at the time of testing. Importantly, the study confirmed that pharmacological action at the GHSR could enhance and attenuate the expression of cue-induced palatable food seeking reinstatement following administration of ghrelin and a GHSR antagonist to the VTA, respectively. The VTA is an area that was previously shown to be important in cue-induced reinstatement of drug seeking (Figure 2).

Together, the results described in Chapters 3 and 6 support the role of ghrelin in the 2 reward-based feeding phenomena investigated in this thesis: dessert consumption and relapse to food seeking. One hypothesis that may be generated from these results is that ghrelin promotes reward-based feeding partly via the direction of instrumental responses in response to conditioned stimuli. Such a scenario would be in line with a number of observations made in both rodents and humans. As mentioned in Chapter 3, pictures of palatable foods increase endogenous ghrelin levels in humans (Schüssler et al., 2012), and ghrelin administration increases the fMRI response to such pictures (Malik et al., 2008). In rats, ghrelin increases (and GHSR antagonism decreases) cue-potentiated feeding (Kanoski et al., 2013; Walker et al., 2012), and endogenous ghrelin levels are correlated with cue-induced cocaine seeking (Tessari et al., 2007). As suggested by Tessari and colleagues (2007), it may be that ghrelin induces a sensitization to reward associated cues, thereby increasing subjects' sensitivity to them. Intriguingly, in a 
Pavlovian-to-Instrumental study, Johnson et al (2009) showed that ghrelin antagonism increases operant responding for sucrose in the presence of food associated conditioned stimuli (Johnson et al., 2009). In the future, studies should focus on resolving ghrelin's exact role in the response to reward cues and characterize the brain areas that support ghrelin's action in this response.

Another area that requires further study involves the compensatory mechanisms that are thought to exist in genetic models of ghrelin signalling disruption. Studies conducted with NPY and AgRP KO models suggest that in the absence of 1 orexigenic system, other orexigenic systems can provide this sort of compensation to manage food intake and body weight. It is unclear however if this results from a greater number of cells that produce other orexigenic signals, or a greater connectivity between the orexigenic centres as well as when during development those changes occur (Grove \& Cowley, 2005). Finally, future studies should continue to characterise the FHH GHSRKO model. 


\section{Conclusion}

Easy access to fat and sugar causes overeating in both rodents and humans, which can lead to obesity (Cornell, Rodin, \& Weingarten, 1989; Valdivia et al., 2014). Food cues, which now dominate our visual landscape more than ever before, can also override internal satiety signals and trigger overeating. Behavioural modifications such as dieting are usually ineffective for sustained weight loss and this is partly due to the enormous rate of relapse to preferred foods in people who are dieting (Wing \& Phelan, 2005). Currently, the most effective weight reduction technique is the gastric bypass surgery, which reduces both circulating ghrelin levels and the desire to eat snacks and desserts (Cummings et al., 2002; le Roux et al., 2011). However, this is an invasive technique associated with some risk and does not represent a practical solution for the majority of the population. In the future, pharmacotherapies that target the ghrelin system to supress reward-based overeating and relapse to high-energy foods during diets may represent an attractive alternative for weight management. While previous attempts to treat obesity via systemic GHSR antagonism have largely been unsuccessful, our results suggest that the VTA may represent a potential pharmaceutical target for these treatments. 


\section{References}

Abbott, A. (2004). The renaissance rat. Nature, 428, 464-466.

Abizaid, A., Liu, Z., Andrews, Z., Shanabrough, M., Borok, E., Elsworth, J., ... Horvath, T. (2006). Ghrelin modulates the activity and synaptic input organization of midbrain dopamine neurons while promoting appetite. The Journal of Clinical Investigation, 116(12), 3229-3239. doi:10.1172/JCI29867

Abizaid, A., Mineur, Y., Roth, R., Elsworth, J., Sleeman, M., Picciotto, M., \& Horvath, T. (2011). Reduced locomotor responses to cocaine in ghrelin-deficient mice. Neuroscience, 192, 500-506. doi:10.1016/j.neuroscience.2011.06.001

Adam, T., \& Epel, E. (2007). Stress, eating and the reward system. Physiology \& Behavior, 91(4), 449-458. doi:10.1016/j.physbeh.2007.04.011

Aguilar, M., Rodríguez-Arias, M., \& Miñarro, J. (2009). Neurobiological mechanisms of the reinstatement of drug-conditioned place preference. Brain Research Reviews, 59(2), 253-277. doi:10.1016/j.brainresrev.2008.08.002

Akanmu, M., \& Honda, K. (2005). Selective stimulation of orexin receptor type 2 promotes wakefulness in freely behaving rats. Brain Research, 1048(1-2), 138-45. doi:10.1016/j.brainres.2005.04.064

Albarran-Zeckler, R., Brantley, A. F., \& Smith, R. (2012). Growth hormone secretagogue receptor (GHS-R1a) knockout mice exhibit improved spatial memory and deficits in contextual memory. Behavioural Brain Research, 232(1), 13-19. doi:10.1016/j.bbr.2012.03.012

Anisman, H., \& Zacharko, R. (1990). Multiple neurochemical and behavioral consequences of stressors: Implications for depression. Pharmacology \& Therapeutics, 46, 119-136.

Arana, F., Parkinson, J., Hinton, E., Holland, A., Owen, A., \& Roberts, A. (2003). Dissociable contributions of the human amygdala and orbitofrontal cortex to incentive motivation and goal selection. Journal of Neuroscience, 23(29), 96329638. Retrieved from http://www.ncbi.nlm.nih.gov/pubmed/14573543

Arase, K., York, D., Shimizu, H., Shargill, N., \& Bray, G. (1988). Effects of corticotropin-releasing factor on food intake and brown adipose tissue thermogenesis in rats. The American Journal of Physiology, 255, 255-259. Retrieved from http://www.ncbi.nlm.nih.gov/pubmed/3262311 
Arvat, E., Maccario, M., Di Vito, L., Broglio, F., Benso, A., Gottero, C., ... Ghigo, E. (2001). Endocrine activities of ghrelin, a natural growth hormone secretagogue (GHS), in humans: comparison and interactions with hexarelin, a nonnatural peptidyl GHS, and GH-releasing hormone. The Journal of Clinical Endocrinology and Metabolism, 86(3), 1169-1174. doi:10.1210/jcem.86.3.7314

Asakawa, A., Inui, A., Kaga, T., Yuzuriha, H., Nagata, T., Fujimiya, M., ... Kasuga, M. (2001). A role of ghrelin in neuroendocrine and behavioral responses to stress in mice. Neuroendocrinology, 74(3), 143-147. doi:54680

Avena, N. M., Rada, P., \& Hoebel, B. G. (2008). Evidence for sugar addiction: behavioral and neurochemical effects of intermittent, excessive sugar intake. Neuroscience and Biobehavioral Reviews, 32(1), 20-39. doi:10.1016/j.neubiorev.2007.04.019

Bagnasco, M., Tulipano, G., Melis, M., Argiolas, A., Cochi, D., \& Muller, E. (2003). Endogenous ghrelin is an orexigenic peptide acting in the arcuate nucleus in response to fasting. Regulatory Peptides, 111(1-3), 161-167. doi:10.1016/S01670115(02)00283-5

Bahi, A., Tolle, V., Fehrentz, J., Brunel, L., Martinez, J., Tomasetto, C., \& Karam, S. (2013). Ghrelin knockout mice show decreased voluntary alcohol consumption and reduced ethanol-induced conditioned place preference. Peptides, 43, 48-55. doi:10.1016/j.peptides.2013.02.008

Baimel, C., Bartlett, S., Chiou, L., Lawrence, A., Muschamp, J., Patkar, O., ... Borgland, S. (2014). Orexin/hypocretin role in reward: implications for opioid and other addictions. British Journal of Pharmacology, in press. doi:10.1111/bph.12639

Banks, W., Tschop, M., Robinson, S., \& Heiman, M. (2002). Extent and Direction of Ghrelin Transport Across the Blood- Brain Barrier Is Determined by Its Unique Primary Structure. Journal of Pharmacology and Experimental Therapeutics, 302(2), 381-388. doi:10.1124/jpet.102.034827.Other

Bari, A., Mar, A., Theobald, D., Elands, S., Oganya, K., Eagle, D., \& Robbins, T. (2011). Prefrontal and monoaminergic contributions to stop-signal task performance in rats. Journal of Neuroscience, 31(25), 9254-9263. doi:10.1523/JNEUROSCI.154311.2011

Bassareo, V., \& Di Chiara, G. (1997). Differential influence of associative and nonassociative learning mechanisms on the responsiveness of prefrontal and accumbal dopamine transmission to food stimuli in rats fed ad libitum. The Journal of Neuroscience, 17(2), 851-861. Retrieved from http://www.ncbi.nlm.nih.gov/pubmed/8987806 
Baxter, M., \& Murray, E. (2002). The amygdala and reward. Nature Reviews: Neuroscience, 3(7), 563-573. doi:10.1038/nrn875

Berridge, K., \& Robinson, T. (1998). What is the role of dopamine in reward: hedonic impact, reward learning, or incentive salience? Brain Research: Brain Research Reviews, 28(3), 309-369. Retrieved from http://www.ncbi.nlm.nih.gov/pubmed/9858756

Berthoud, H. (2004). Mind versus metabolism in the control of food intake and energy balance. Physiology \& Behavior, 81(5), 781-93. doi:10.1016/j.physbeh.2004.04.034

Blum, I., Patterson, Z., Khazall, R., Lamont, E., Sleeman, M., Horvath, T., \& Abizaid, A. (2009). Reduced anticipatory locomotor responses to scheduled meals in ghrelin receptor deficient mice. Neuroscience, 164(2), 351-359.

doi:10.1016/j.neuroscience.2009.08.009

Borgland, S., Chang, S., Bowers, M., Thompson, J., Vittoz, N., Floresco, S., ... Bonci, A. (2009). Orexin A/hypocretin-1 selectively promotes motivation for positive reinforcers. The Journal of Neuroscience, 29(36), 11215-11225. doi:10.1523/JNEUROSCI.6096-08.2009

Borgland, S., Malenka, R., \& Bonci, A. (2004). Acute and chronic cocaine-induced potentiation of synaptic strength in the ventral tegmental area: electrophysiological and behavioral correlates in individual rats. The Journal of Neuroscience, 24(34), 7482-7490. doi:10.1523/JNEUROSCI.1312-04.2004

Bossert, J., Liu, S., Lu, L., \& Shaham, Y. (2004). A role of ventral tegmental area glutamate in contextual cue-induced relapse to heroin seeking. Journal of Neuroscience, 24(47), 10726-10730. doi:10.1523/JNEUROSCI.3207-04.2004

Boulougouris, V., Glennon, J., \& Robbins, T. (2008). Dissociable effects of selective 5HT2A and 5-HT2C receptor antagonists on serial spatial reversal learning in rats. Neuropsychopharmacology, 33(8), 2007-2019. doi:10.1038/sj.npp.1301584

Boutrel, B., Kenny, P., Specio, S., Martin-Fardon, R., Markou, A., Koob, G., \& de Lecea, L. (2005). Role for hypocretin in mediating stress-induced reinstatement of cocaineseeking behavior. PNAS, 102(52), 19168-19173. doi:10.1073/pnas.0507480102

Bray, G., Nielsen, S., \& Popkin, B. (2004). Consumption of high-fructose corn syrup in beverages may play a role in the epidemic of obesity. The American Journal of Clinical Nutrition, 79(4), 537-43. Retrieved from http://www.ncbi.nlm.nih.gov/pubmed/15051594

Brown, R., Khoo, S., \& Lawrence, A. (2013). Central orexin (hypocretin) 2 receptor antagonism reduces ethanol self-administration, but not cue-conditioned ethanol- 
seeking, in ethanol-preferring rats. The International Journal of Neuropsychopharmacology, 16(9), 2067-2079. doi:10.1017/S1461145713000333

Bülbül, M., Babygirija, R., Zheng, J., Ludwig, K., Xu, H., Lazar, J., \& Takahashi, T. (2011). Food intake and interdigestive gastrointestinal motility in ghrelin receptor mutant rats. Journal of Gastroenterology, 46(4), 469-478. doi:10.1007/s00535-0100366-6

Cahill, S., Hatchard, T., Abizaid, A., \& Holahan, M. (2014). An examination of early neural and cognitive alterations in hippocampal-spatial function of ghrelin receptordeficient rats. Behavioural Brain Research, 264, 105-115.

doi:10.1016/j.bbr.2014.02.004

Cardinal, R., Parkinson, J., Hall, J., \& Everitt, B. (2002). Emotion and motivation: the role of the amygdala, ventral striatum, and prefrontal cortex. Neuroscience and Biobehavioral Reviews, 26(3), 321-352. Retrieved from http://www.ncbi.nlm.nih.gov/pubmed/12034134

Carlini, V., Monzón, M., Varas, M., Cragnolini, A., Schiöth, H., Scimonelli, T., \& de Barioglio, S. (2002). Ghrelin increases anxiety-like behavior and memory retention in rats. Biochemical and Biophysical Research Communications, 299(5), 739-743. Retrieved from http://www.ncbi.nlm.nih.gov/pubmed/12470640

Carlini, V., Varas, M., Cragnolini, A., Schiöth, H., Scimonelli, T., \& de Barioglio, S. (2004). Differential role of the hippocampus, amygdala, and dorsal raphe nucleus in regulating feeding, memory, and anxiety-like behavioral responses to ghrelin. Biochemical and Biophysical Research Communications, 313(3), 635-641. doi:10.1016/j.bbrc.2003.11.150

Carr, K. (2007). Chronic food restriction: enhancing effects on drug reward and striatal cell signaling. Physiology \& Behavior, 91(5), 459-472.

doi:10.1016/j.physbeh.2006.09.021

Carvajal, P., Carlini, V., Schiöth, H., de Barioglio, S., \& Salvatierra, N. (2009). Central ghrelin increases anxiety in the Open Field test and impairs retention memory in a passive avoidance task in neonatal chicks. Neurobiology of Learning and Memory, 91(4), 402-407. doi:10.1016/j.nlm.2008.12.008

Choi, D., Davis, J., Fitzgerald, M., \& Benoit, S. (2010). The role of orexin-A in food motivation, reward-based feeding behavior and food-induced neuronal activation in rats. Neuroscience, 167(1), 11-20. doi:10.1016/j.neuroscience.2010.02.002

Chuang, J., Perello, M., Sakata, I., Osborne-Lawrence, S., Savitt, J., Lutter, M., \& Zigman, J. (2011). Ghrelin mediates stress-induced food-reward behavior in mice. The Journal of Clinical Investigation, 121(7), 2684-2692. doi:10.1172/JCI57660.2684 
Chuang, J., \& Zigman, J. (2010). Ghrelin's Roles in Stress, Mood, and Anxiety Regulation. International Journal of Peptides, 2010, 460549.

doi:10.1155/2010/460549

Cippitelli, A., Karlsson, C., Shaw, J., Thorsell, A., Gehlert, D., \& Heilig, M. (2010).

Suppression of alcohol self-administration and reinstatement of alcohol seeking by melanin-concentrating hormone receptor $1(\mathrm{MCH} 1-\mathrm{R})$ antagonism in Wistar rats. Psychopharmacology, 211(4), 367-75. doi:10.1007/s00213-010-1891-y

Clarke, H., Dalley, J., Crofts, H., Robbins, T., \& Roberts, A. (2004). Cognitive inflexibility after prefrontal serotonin depletion. Science, 304(5672), 878-880. doi:10.1126/science. 1094987

Clifford, P., Rodriguez, J., Schul, D., Hughes, S., Kniffin, T., Hart, N., ... Wellman, P. (2012). Attenuation of cocaine-induced locomotor sensitization in rats sustaining genetic or pharmacologic antagonism of ghrelin receptors. Addiction Biology, 17(6), 956-963. doi:10.1111/j.1369-1600.2011.00339.x

Clifford, S., Zeckler, R., Buckman, S., Thompson, J., Hart, N., Wellman, P., \& Smith, R. (2011). Impact of food restriction and cocaine on locomotion in ghrelin- and ghrelin-receptor knockout mice. Addiction Biology, 16(3), 386-392. doi:10.1111/j.1369-1600.2010.00253.x

Cone, J., McCutcheon, J., \& Roitman, M. (2014). Ghrelin acts as an interface between physiological state and phasic dopamine signaling. Journal of Neuroscience, 34(14), 4905-4913. doi:10.1523/JNEUROSCI.4404-13.2014

Connor, D., Jones, F., Conner, M., Mcmillan, B., \& Ferguson, E. (2008). Effects of Daily Hassles and Eating Style on Eating Behavior. Health Psychology, 27(S1), 20-31. doi:10.1037/0278-6133.27.1(Suppl.).S20

Contarino, A., Dellu, F., Koob, G., Smith, G., Lee, K., Vale, W., \& Gold, L. (2000). Dissociation of locomotor activation and suppression of food intake induced by CRF in CRFR1-deficient mice. Endocrinology, 141(7), 2698-2702.

Cornell, C., Rodin, J., \& Weingarten, H. (1989). Stimulus-induced eating when satiated. Physiology \& Behavior, 45, 695-704.

Corwin, R., \& Grigson, P. (2009). Symposium Overview — Food Addiction : Fact or Fiction? The Journal of Nutrition, 139(4), 617-619. doi:10.3945/jn.108.097691.Is

Costall, B., Jones, B., Kelly, M., Naylor, R., \& Tomkins, D. (1989). Exploration of mice in a black and white test box: validation as a model of anxiety. Pharmacology Biochemistry and Behavior, 32, 777-785. 
Cowley, M., Smith, R., Diano, S., Tschöp, M., Pronchuk, N., Grove, K., ... Horvath, T. (2003). The distribution and mechanism of action of ghrelin in the CNS demonstrates a novel hypothalamic circuit regulating energy homeostasis. Neuron, 37(4), 649-661. Retrieved from http://www.ncbi.nlm.nih.gov/pubmed/12597862

Cummings, D., Frayo, R., Marmonier, C., Aubert, R., \& Chapelot, D. (2004). Plasma ghrelin levels and hunger scores in humans initiating meals voluntarily without time- and food-related cues. American Journal of Physiology. Endocrinology and Metabolism, 287(2), 297-304. doi:10.1152/ajpendo.00582.2003

Cummings, D., Purnell, J., Frayo, R., Schmidova, K., Wisse, B., \& Weigle, D. (2001). A preprandial rise in plasma ghrelin levels suggests a role in meal initiation in humans. Diabetes, 50(8), 1714-1719. Retrieved from http://www.ncbi.nlm.nih.gov/pubmed/11473029

Cummings, D., Weigle, D., Frayo, R., Breen, P., Ma, M., Dellinger, E., \& Purnell, J. (2002). Plasma ghrelin levels after diet-induced weight loss or gastric bypass surgery. The New England Journal of Medicine, 346(21), 1623-1630. doi:10.1056/NEJMoa012908

Currie, P., Coiro, C., Duenas, R., Guss, J., Mirza, A., \& Tal, N. (2011). Urocortin I inhibits the effects of ghrelin and neuropeptide $\mathrm{Y}$ on feeding and energy substrate utilization. Brain Research, 1385, 127-134. doi:10.1016/j.brainres.2011.01.114

Currie, P., Khelemsky, R., Rigsbee, E., Dono, L., Coiro, C., Chapman, C., \& Hinchcliff, K. (2012). Ghrelin is an orexigenic peptide and elicits anxiety-like behaviors following administration into discrete regions of the hypothalamus. Behavioural Brain Research, 226(1), 96-105. doi:10.1016/j.bbr.2011.08.037

Dalley, J. W., Cardinal, R. N., \& Robbins, T. W. (2004). Prefrontal executive and cognitive functions in rodents: neural and neurochemical substrates. Neuroscience \& Biobehavioral Reviews, 28(7), 771-784. doi:10.1016/j.neubiorev.2004.09.006

Dallman, M. (2010). Stress-induced obesity and the emotional nervous system. Trends in Endocrinology and Metabolism, 21(3), 159-165. doi:10.1016/j.tem.2009.10.004.Stress-induced

Dallman, M., Pecoraro, N., Akana, S., La Fleur, S., Gomez, F., Houshyar, H., ... Manalo, S. (2003). Chronic stress and obesity: a new view of "comfort food." PNAS, 100(20), 11696-701. doi:10.1073/pnas.1934666100

Dallman, M., Pecoraro, N., \& la Fleur, S. (2005). Chronic stress and comfort foods: selfmedication and abdominal obesity. Brain, Behavior, and Immunity, 19(4), 275-280. doi:10.1016/j.bbi.2004.11.004 
Davis, J., Choi, D., Clegg, D., \& Benoit, S. (2011). Signaling through the ghrelin receptor modulates hippocampal function and meal anticipation in mice. Physiology \& Behavior, 103(1), 39-43. doi:10.1016/j.physbeh.2010.10.017

Davis, J., Perello, M., Choi, D. L., Magrisso, I., Kirchner, H., Pfluger, P., ... Benoit, S. (2012). GOAT induced ghrelin acylation regulates hedonic feeding. Hormones and Behavior, 62(5), 598-604. doi:10.1016/j.yhbeh.2012.08.009

Davis, K., Wellman, P., \& Clifford, P. (2007). Augmented cocaine conditioned place preference in rats pretreated with systemic ghrelin. RegulatoryPpeptides, 140(3), 148-152. doi:10.1016/j.regpep.2006.12.003

De Smet, B., Mitselos, A., \& Depoortere, I. (2009). Motilin and ghrelin as prokinetic drug targets. Pharmacology \& Therapeutics, 123(2), 207-223.

doi:10.1016/j.pharmthera.2009.04.004

De Wit, H., \& Stewart, J. (1981). Psychopharmacology Reinstatement of CocaineReinforced Responding in the Rat. Psychopharmacology, 75(2), 134-143.

Depke, M., Fusch, G., Domanska, G., Geffers, R., Völker, U., Schuett, C., \& Kiank, C. (2008). Hypermetabolic syndrome as a consequence of repeated psychological stress in mice. Endocrinology, 149(6), 2714-2723. doi:10.1210/en.2008-0038

Depoortere, I., Thijs, T., \& Peeters, T. (2006). The contractile effect of the ghrelin receptor antagonist, D-Lys3-GHRP-6, in rat fundic strips is mediated through 5-HT receptors. European Journal of Pharmacology, 537(1-3), 160-165. doi:10.1016/j.ejphar.2006.03.043

Di Sebastiano, A., Wilson-Pérez, H., Lehman, M., \& Coolen, L. (2011). Lesions of orexin neurons block conditioned place preference for sexual behavior in male rats. Hormones and Behavior, 59(1), 1-8. doi:10.1016/j.yhbeh.2010.09.006

Diano, S., Farr, S., Benoit, S., McNay, E., da Silva, I., Horvath, B., ... Horvath, T. (2006). Ghrelin controls hippocampal spine synapse density and memory performance. Nature Neuroscience, 9(3), 381-388. doi:10.1038/nn1656

Disse, A., Bussier, A., Veyrat-Durebex, C., Deblon, N., Pfluger, P., Tschöp, M., ... Rohner-Jeanrenaud, F. (2010). Peripheral ghrelin enhances sweet taste food consumption and preference, regardless of its caloric content. Physiology \& Behavior, 101(2), 277-281. doi:10.1016/j.physbeh.2010.05.017

Drazen, D., Vahl, T., D’Alessio, D., Seeley, R., \& Woods, S. (2006). Effects of a fixed meal pattern on ghrelin secretion: evidence for a learned response independent of nutrient status. Endocrinology, 147(1), 23-30. doi:10.1210/en.2005-0973 
Drewnowski, A., \& Specter, S. (2004). Poverty and obesity: the role of energy density and energy costs. The American Journal of Clinical Nutrition, 79(1), 6-16.

Retrieved from http://www.ncbi.nlm.nih.gov/pubmed/14684391

Eagle, D., \& Baunez, C. (2010). Is there an inhibitory-response-control system in the rat? Evidence from anatomical and pharmacological studies of behavioral inhibition. Neuroscience and Biobehavioral Reviews, 34(1), 50-72. doi:10.1016/j.neubiorev.2009.07.003

Egecioglu, E., Jerlhag, E., Salomé, N., Skibicka, K., Haage, D., Bohlooly-Y, M., ... Dickson, S. (2010). Ghrelin increases intake of rewarding food in rodents. Addiction Biology, 15(3), 304-11. doi:10.1111/j.1369-1600.2010.00216.x

Elfhag, K., \& Rössner, S. (2005). Who succeeds in maintaining weight loss? A conceptual review of factors associated with weight loss maintenance and weight regain. Obesity Reviews, 6(1), 67-85. doi:10.1111/j.1467-789X.2005.00170.x

Elliott, S., Keim, N., Stern, J., Teff, K., \& Havel, P. (2002). Fructose, weight gain, and the insulin resistance syndrome. The American Journal of Clinical Nutrition, 76(5), 911-22. Retrieved from http://www.ncbi.nlm.nih.gov/pubmed/12399260

Epel, E., Lapidus, R., McEwen, B., \& Brownell, K. (2001). Stress may add bite to appetite in women: a laboratory study of stress-induced cortisol and eating behavior. Psychoneuroendocrinology, 26(1), 37-49. Retrieved from http://www.ncbi.nlm.nih.gov/pubmed/11070333

Epstein, D., Preston, K., Stewart, J., \& Shaham, Y. (2006). Toward a model of drug relapse: an assessment of the validity of the reinstatement procedure. Psychopharmacology, 189(1), 1-16. doi:10.1007/s00213-006-0529-6

Esposito, M., Pellinen, J., Kapás, L., \& Szentirmai, E. (2012). Impaired wake-promoting mechanisms in ghrelin receptor-deficient mice. The EuropeanJournal of Neuroscience, 35(2), 233-243. doi:10.1111/j.1460-9568.2011.07946.x

Everitt, B., Cardinal, R., Parkinson, J., \& Robbins, T. (2003). Impact of AmygdalaDependent Mechanisms of Emotional Learning. Annals of the New York Academy of Sciences, 985, 233-250.

Fedoroff, I., Polivy, J., \& Herman, C. (1997). The effect of pre-exposure to food cues on the eating behavior of restrained and unrestrained eaters. Appetite, 28(1), 33-47. Retrieved from http://www.ncbi.nlm.nih.gov/pubmed/9134093

Finger, B., Dinan, T., \& Cryan, J. (2012). Diet-induced obesity blunts the behavioural effects of ghrelin: studies in a mouse-progressive ratio task. Psychopharmacology, 220(1), 173-181. doi:10.1007/s00213-011-2468-0 
Floresco, S., McLaughlin, R., \& Haluk, D. (2008). Opposing roles for the nucleus accumbens core and shell in cue-induced reinstatement of food-seeking behavior. Neuroscience, 154(3), 877-884. doi:10.1016/j.neuroscience.2008.04.004

Fuchs, R., Evans, K., Parker, M., \& See, R. (2004). Differential involvement of the core and shell subregions of the nucleus accumbens in conditioned cue-induced reinstatement of cocaine seeking in rats. Psychopharmacology, 176(3-4), 459-465. doi:10.1007/s00213-004-1895-6

Fulton, S. (2000). Modulation of Brain Reward Circuitry by Leptin. Science, 287(5450), 125-128. doi:10.1126/science.287.5450.125

Fulton, S. (2010). Appetite and reward. Frontiers in Neuroendocrinology, 31(1), 85-103. doi:10.1016/j.yfrne.2009.10.003

Furness, J., Hunne, B., Matsuda, N., Yin, L., Russo, D., Kato, I., ... Bron, R. (2011). Investigation of the presence of ghrelin in the central nervous system of the rat and mouse. Neuroscience, 193, 1-9. doi:10.1016/j.neuroscience.2011.07.063

Gallagher, M., \& Holland, P. (1994). Review The amygdala complex : Multiple roles in associative learning and attention. PNAS, 91, 11771-11776.

Gao, Q., \& Horvath, T. (2007). Neurobiology of feeding and energy expenditure. Annual Review of Neuroscience, 30, 367-98. doi:10.1146/annurev.neuro.30.051606.094324

Ghigo, E., Broglio, F., Arvat, E., Maccario, M., Papotti, M., \& Muccioli, G. (2005). Ghrelin: more than a natural GH secretagogue and/or an orexigenic factor. Clinical Endocrinology, 62(1), 1-17. doi:10.1111/j.1365-2265.2004.02160.x

Ghitza, U., Gray, S., Epstein, D., Rice, K., \& Shaham, Y. (2006). The anxiogenic drug yohimbine reinstates palatable food seeking in a rat relapse model: a role of CRF1 receptors. Neuropsychopharmacology, 31(10), 2188-2196. doi:10.1038/sj.npp.1300964

Ghitza, U., Nair, S., Golden, S., Gray, S., Uejima, J., Bossert, J., \& Shaham, Y. (2007). Peptide YY3-36 decreases reinstatement of high-fat food seeking during dieting in a rat relapse model. The Journal of Neuroscience, 27(43), 11522-11532. doi:10.1523/JNEUROSCI.5405-06.2007

Gorin, A., Phelan, S., Wing, R., \& Hill, J. (2004). Promoting long-term weight control: does dieting consistency matter? International Journal of Obesity, 28(2), 278-281. doi:10.1038/sj.ijo.0802550

Greeno, C., \& Wing, R. (1994). Stress-induced eating. Psychological Bulletin, 115(3), 444-464. 
Grilo, C., Shiffman, S., \& Wing, R. (1989). Relapse crises and coping among dieters. Journal of Consulting and Clinical Psychology, 57(4), 488-495. Retrieved from http://www.ncbi.nlm.nih.gov/pubmed/2768608

Grimm, J. W., Hope, B. T., Wise, R. A., \& Shaham, Y. (2001). Incubation of cocaine craving after withdrawal. Nature, 412(July), 141-142.

Grove, K. L., \& Cowley, M. A. (2005). Is ghrelin a signal for the development of metabolic systems ?, 115(12), 3393-3397. doi:10.1172/JCI27211.response

Guan, X., Yu, H., Palyha, O., McKee, K., Feighner, S., Sirinathsinghji, D., ... Howard, A. (1997). Distribution of mRNA encoding the growth hormone secretagogue receptor in brain and peripheral tissues. Molecular Brain Research, 48(1), 23-29. Retrieved from http://www.ncbi.nlm.nih.gov/pubmed/9379845

Haber, S., Fudge, J., \& McFarland, N. (2000). Striatonigrostriatal pathways in primates form an ascending spiral from the shell to the dorsolateral striatum. The Journal of Neuroscience, 20(6), 2369-2382. Retrieved from http://www.ncbi.nlm.nih.gov/pubmed/10704511

Hamlin, A., Clemens, K., \& McNally, G. (2008). Renewal of extinguished cocaineseeking. Neuroscience, 151(3), 659-670. doi:10.1016/j.neuroscience.2007.11.018

Hansson, C., Shirazi, R., Näslund, J., Vogel, H., Neuber, C., Holm, G., ... Skibicka, K. (2012). Ghrelin influences novelty seeking behavior in rodents and men. PloS One, 7(12), 50409. doi:10.1371/journal.pone.0050409

Harris, G. C., \& Aston-Jones, G. (2006). Arousal and reward: a dichotomy in orexin function. Trends in Neurosciences, 29(10), 571-577. doi:10.1016/j.tins.2006.08.002

Harris, G., Wimmer, M., \& Aston-Jones, G. (2005). A role for lateral hypothalamic orexin neurons in reward seeking. Nature, 437(7058), 556-559. doi:10.1038/nature04071

Harris, G., Wimmer, M., Randall-Thompson, J., \& Aston-Jones, G. (2007). Lateral hypothalamic orexin neurons are critically involved in learning to associate an environment with morphine reward. Behavioural Brain Research, 183(1), 43-51. doi:10.1016/j.bbr.2007.05.025

Highfield, D., Clements, A., Shalev, U., McDonald, R., Featherstone, R., Stewart, J., \& Shaham, Y. (2000). Involvement of the medial septum in stress-induced relapse to heroin seeking in rats. The European Journal of Neuroscience, 12(5), 1705-1713. Retrieved from http://www.ncbi.nlm.nih.gov/pubmed/10792448

Holland, P., \& Gallagher, M. (2003). Double dissociation of the effects of lesions of basolateral and central amygdala on conditioned stimulus-potentiated feeding and 
Pavlovian-instrumental transfer. European Journal of Neuroscience, 17(8), 16801694. doi:10.1046/j.1460-9568.2003.02585.x

Holst, B., \& Schwartz, T. (2004). Constitutive ghrelin receptor activity as a signaling setpoint in appetite regulation. Trends in Pharmacological Sciences, 25(3), 113-117. doi:10.1016/j.tips.2004.01.010

Hoover, W., \& Vertes, R. (2007). Anatomical analysis of afferent projections to the medial prefrontal cortex in the rat. Brain Structure \& Function, 212(2), 149-179. doi:10.1007/s00429-007-0150-4

Horvath, T., Diano, S., Sotonyi, P., Heiman, M., \& Tschöp, M. (2001). Minireview: ghrelin and the regulation of energy balance -a hypothalamic perspective. Endocrinology, 142(10), 4163-4169. doi:10.1210/endo.142.10.8490

Hosoda, H., Kojima, M., \& Kangawa, K. (2002). Ghrelin and the regulation of food intake and energy balance. Molecular Interventions, 2(8), 494-503. doi:10.1124/mi.2.8.494

Ito, R., Dalley, J., Howes, S., Robbins, T., \& Everitt, B. (2000). Dissociation in conditioned dopamine release in the nucleus accumbens core and shell in response to cocaine cues and during cocaine-seeking behavior in rats. The Journal of Neuroscience, 20(19), 7489-7495. Retrieved from http://www.ncbi.nlm.nih.gov/pubmed/11007908

Ito, R., Robbins, T., \& Everitt, B. (2004). Differential control over cocaine-seeking behavior by nucleus accumbens core and shell. Nature Neuroscience, 7(4), 389-397. doi:10.1038/nn1217

Jacob, H. (1999). Functional genomics and rat models. Genome Research, 9(11), 10131016. doi:10.1101/gr.9.11.1013

James, M., Charnley, J., Levi, E., Jones, E., Yeoh, J., Smith, D., \& Dayas, C. (2011). Orexin-1 receptor signalling within the ventral tegmental area, but not the paraventricular thalamus, is critical to regulating cue-induced reinstatement of cocaine-seeking. International Journal of Neuropsychopharmacology, 14(5), 684690. doi:10.1017/S1461145711000423

Jászberényi, M., Bujdosó, E., Bagosi, Z., \& Telegdy, G. (2006). Mediation of the behavioral, endocrine and thermoregulatory actions of ghrelin. Hormones and Behavior, 50(2), 266-273. doi:10.1016/j.yhbeh.2006.03.010

Jerlhag, E. (2008). Systemic administration of ghrelin induces conditioned place preference and stimulates accumbal dopamine. Addiction Biology, 13(3-4), 358-563. doi:10.1111/j.1369-1600.2008.00125.x 
Jerlhag, E., Egecioglu, E., Dickson, S., Andersson, M., Svensson, L., \& Engel, J. (2006). Ghrelin stimulates locomotor activity and accumbal dopamine-overflow via central cholinergic systems in mice : implications for its involvement in brain reward. Addiction Biology, 11(1), 45-54. doi:10.1111/j.1355-6215.2006.00002.x

Jerlhag, E., Egecioglu, E., Dickson, S., Douhan, A., Svensson, L., \& Engel, J. (2007). Ghrelin administration into tegmental areas stimulates locomotor activity and increases extracellular concentration of dopamine in the nucleus accumbens. Addiction Biology, 12(1), 6-16. doi:10.1111/j.1369-1600.2006.00041.x

Jerlhag, E., Egecioglu, E., Dickson, S., \& Engel, J. (2010). Ghrelin receptor antagonism attenuates cocaine- and amphetamine-induced locomotor stimulation, accumbal dopamine release, and conditioned place preference. Psychopharmacology, 211(4), 415-422. doi:10.1007/s00213-010-1907-7

Jerlhag, E., Egecioglu, E., Dickson, S., \& Engel, J. (2011). Glutamatergic regulation of ghrelin-induced activation of the mesolimbic dopamine system. Addiction Biology, 16(1), 82-91. doi:10.1111/j.1369-1600.2010.00231.x

Jerlhag, E., Egecioglu, E., Landgren, S., Salome, N., Heilig, M., Moechars, D., ... Engel, J. (2009). Requirement of central ghrelin signaling for alcohol reward. PNAS, 106(27), 11318-11321.

Jerlhag, E., \& Engel, J. a. (2011). Ghrelin receptor antagonism attenuates nicotineinduced locomotor stimulation, accumbal dopamine release and conditioned place preference in mice. Drug and Alcohol Dependence, 117(2-3), 126-31. doi:10.1016/j.drugalcdep.2011.01.010

Jerlhag, E., Landgren, S., Egecioglu, E., Dickson, S., \& Engel, J. (2011). The alcoholinduced locomotor stimulation and accumbal dopamine release is suppressed in ghrelin knockout mice. Alcohol, 45(4), 341-347. doi:10.1016/j.alcohol.2010.10.002

Jhou, T., Fields, H., Baxter, M., Saper, C., \& Holland, P. (2009). The rostromedial tegmental nucleus (RMTg), a GABAergic afferent to midbrain dopamine neurons, encodes aversive stimuli and inhibits motor responses. Neuron, 61(5), 786-800. doi:10.1016/j.neuron.2009.02.001

Jiang, H., Betancourt, L., \& Smith, R. (2006). Ghrelin amplifies dopamine signaling by cross talk involving formation of growth hormone secretagogue receptor/dopamine receptor subtype 1 heterodimers. Molecular Endocrinology, 20(8), 1772-1785. doi:10.1210/me.2005-0084

Johnson, A., Canter, R., Gallagher, M., \& Holland, P. (2009). Assessing the role of the growth hormone secretagogue receptor in motivational learning and food intake. Behavioral Neuroscience, 123(5), 1058-1065. doi:10.1037/a0016808 
Johnson, B., \& North, R. (1992). Two types of neurone in the rat ventral tegmental area and their synaptic inputs. Journal of Physiology, 450, 455-468.

Kalivas, P., \& McFarland, K. (2003). Brain circuitry and the reinstatement of cocaineseeking behavior. Psychopharmacology, 168(1-2), 44-56. doi:10.1007/s00213-0031393-2

Kalivas, P., \& Volkow, N. (2005). The Neural Basis of Addiction : A Pathology of Motivation and Choice. American Journal of Psychiatry, 162(8), 1403-1413.

Kandiah, J., Yake, M., Jones, J., \& Meyer, M. (2006). Stress influences appetite and comfort food preferences in college women. Nutrition Research, 26(3), 118-123. doi:10.1016/j.nutres.2005.11.010

Kanoski, S., Fortin, S., Ricks, K., \& Grill, H. (2013). Ghrelin signaling in the ventral hippocampus stimulates learned and motivational aspects of feeding via PI3K-Akt signaling. Biological Psychiatry, 73(9), 915-923.

doi:10.1016/j.biopsych.2012.07.002

Kayman, S., Bruvold, W., \& Stern, J. (1990). Maintenance and relapse after weight loss in women: behavioral aspects. American Journal of Clinical Nutrition, 52, 800-807.

Keen-Rhinehart, E., \& Bartness, T. (2005). Peripheral ghrelin injections stimulate food intake, foraging, and food hoarding in Siberian hamsters. American Journal of Physiology Regulatory, Integrative and Comparative Physiology, 288, 716-722. doi:10.1152/ajpregu.00705.2004.

Kelley, A. (2004). Ventral striatal control of appetitive motivation: role in ingestive behavior and reward-related learning. Neuroscience and Biobehavioral Reviews, 27(8), 765-776. doi:10.1016/j.neubiorev.2003.11.015

Kelley, A., Bakshi, V., Fleming, S., \& Holahan, M. (2000). A pharmacological analysis of the substrates underlying conditioned feeding induced by repeated opioid stimulation of the nucleus accumbens. Neuropsychopharmacology, 23(4), 455-467. doi:10.1016/S0893-133X(00)00117-2

Kenny, P. (2011). Reward mechanisms in obesity: new insights and future directions. Neuron, 69(4), 664-679. doi:10.1016/j.neuron.2011.02.016

Killgore, W., Young, A., Femia, L., Bogorodzki, P., Rogowska, J., \& Yurgelun-Todd, D. (2003). Cortical and limbic activation during viewing of high- versus low-calorie foods. NeuroImage, 19(4), 1381-1394. doi:10.1016/S1053-8119(03)00191-5

King, S. J., Isaacs, A., O'Farrell, E., \& Abizaid, A. (2011). Motivation to obtain preferred foods is enhanced by ghrelin in the ventral tegmental area. Hormones and Behavior, 60(5), 572-580. doi:10.1016/j.yhbeh.2011.08.006 
Kolb, B. (1984). Functions of the frontal cortex of the rat: a comparative review. Brain Research, 320(1), 65-98. Retrieved from http://www.ncbi.nlm.nih.gov/pubmed/6440660

Koob, G., \& Volkow, N. (2010). Neurocircuitry of addiction. Neuropsychopharmacology, 35(1), 217-238. doi:10.1038/npp.2009.110

Koopmann, A., von der Goltz, C., Grosshans, M., Dinter, C., Vitale, M., Wiedemann, K., $\&$ Kiefer, F. (2012). The association of the appetitive peptide acetylated ghrelin with alcohol craving in early abstinent alcohol dependent individuals.

Psychoneuroendocrinology, 37(7), 980-986. doi:10.1016/j.psyneuen.2011.11.005

Korotkova, T., Sergeeva, O., Eriksson, K., Haas, H., \& Brown, R. (2003). Excitation of ventral tegmental area dopaminergic and nondopaminergic neurons by orexins/hypocretins. Journal of Neuroscience, 23(1), 7-11. Retrieved from http://www.ncbi.nlm.nih.gov/pubmed/12514194

Koya, E., Uejima, J., Wihbey, K., Bossert, J., Hope, B., \& Shaham, Y. (2009). Role of ventral medial prefrontal cortex in incubation of cocaine craving. Neuropharmacology, 56(S1), 177-185. doi:10.1016/j.neuropharm.2008.04.022

Kramer, F., Jeffery, R., Forster, J., \& Snell, M. (1989). Long-term follow-up of behavioral treatment for obesity: patterns of weight regain among men and women. International Journal of Obesity, 13(2), 123-136. Retrieved from http://www.ncbi.nlm.nih.gov/pubmed/2663745

Krettek, J., \& Price, J. (1977). Projections from the amygdaloid complex to the cerebral cortex and thalamus in the rat and cat. The Journal of Comparative Neurology, 172(4), 687-722. doi:10.1002/cne.901720408

Kristensson, E., Sundqvist, M., Håkanson, R., \& Lindström, E. (2007). High gastrin cell activity and low ghrelin cell activity in high-anxiety Wistar Kyoto rats. The Journal of Endocrinology, 193(2), 245-250. doi:10.1677/JOE-07-0028

Kristenssson, E., Sundqvist, M., Astin, M., Kjerling, M., Mattsson, H., Dornonville de la Cour, C., ... Lindström, E. (2006). Acute psychological stress raises plasma ghrelin in the rat. Regulatory Peptides, 134(2-3), 114-117.

doi:10.1016/j.regpep.2006.02.003

Krügel, U., Schraft, T., Kittner, H., Kiess, W., \& Illes, P. (2003). Basal and feedingevoked dopamine release in the rat nucleus accumbens is depressed by leptin. European Journal of Pharmacology, 482(1-3), 185-187. doi:10.1016/j.ejphar.2003.09.047 
Kupferschmidt, D., Tribe, E., \& Erb, S. (2009). Effects of repeated yohimbine on the extinction and reinstatement of cocaine seeking. Pharmacology, Biochemistry, and Behavior, 91(3), 473-480. doi:10.1016/j.pbb.2008.08.026

Landgren, S., Engel, J., Hyytiä, P., Zetterberg, H., Blennow, K., \& Jerlhag, E. (2011). Expression of the gene encoding the ghrelin receptor in rats selected for differential alcohol preference. Behavioural Brain Research, 221(1), 182-188. doi:10.1016/j.bbr.2011.03.003

Landgren, S., Simms, J., Hyytiä, P., Engel, J., Bartlett, S., \& Jerlhag, E. (2012). Ghrelin receptor (GHS-R1A) antagonism suppresses both operant alcohol selfadministration and high alcohol consumption in rats. Addiction Biology, 17(1), 8694. doi:10.1111/j.1369-1600.2010.00280.x

Landgren, S., Simms, J., Thelle, D., Strandhagen, E., Bartlett, S., Engel, J., \& Jerlhag, E. (2011). The ghrelin signalling system is involved in the consumption of sweets. PloS One, 6(3), 18170. doi:10.1371/journal.pone.0018170

Lawrence, C., Snape, A., Baudoin, F., \& Luckman, S. (2002). Acute central ghrelin and $\mathrm{GH}$ secretagogues induce feeding and activate brain appetite centers. Endocrinology, 143(1), 155-162. doi:10.1210/endo.143.1.8561

Lê, A., Quan, B., Juzytch, W., Fletcher, P., Joharchi, N., \& Shaham, Y. (1998). Reinstatement of alcohol-seeking by priming injections of alcohol and exposure to stress in rats. Psychopharmacology, 135(2), 169-174. Retrieved from http://www.ncbi.nlm.nih.gov/pubmed/9497022

Le Roux, C., Bueter, M., Theis, N., Werling, M., Ashrafian, H., Löwenstein, C., ... Lutz, T. (2011). Gastric bypass reduces fat intake and preference. American Journal of Physiology. Regulatory, Integrative and Comparative Physiology, 301, 1057-1066. doi:10.1152/ajpregu.00139.2011.

Leggio, L., Zywiak, W., Fricchione, S., Edwards, S., de la Monte, S., Swift, R., \& Kenna, G. (2014). Intravenous Ghrelin Administration Increases Alcohol Craving in Alcohol-Dependent Heavy Drinkers: A Preliminary Investigation. Biological Psychiatry, in press. doi:10.1016/j.biopsych.2014.03.019

Lenoir, M., Serre, F., Cantin, L., \& Ahmed, S. (2007). Intense sweetness surpasses cocaine reward. PloS One, 2(8), e698. doi:10.1371/journal.pone.0000698

LeSauter, J., Hoque, N., Weintraub, M., Pfaff, D., \& Silver, R. (2009). Stomach ghrelinsecreting cells as food-entrainable circadiam clocks. PNAS, 106(32), 13582-13587.

Li, Y., Li, F., Wang, X., Wu, P., Zhao, M., Xu, C., ... Lu, L. (2008). Central amygdala extracellular signal-regulated kinase signaling pathway is critical to incubation of 
opiate craving. Journal of Neuroscience, 28(49), 13248-13257.

doi:10.1523/JNEUROSCI.3027-08.2008

Lowe, M., \& Butryn, M. (2007). Hedonic hunger: a new dimension of appetite? Physiology \& Behavior, 91(4), 432-439. doi:10.1016/j.physbeh.2007.04.006

Lu, S., Guan, J., Wang, Q., Uehara, K., Yamada, S., Goto, N., ... Shioda, S. (2002). Immunocytochemical observation of ghrelin-containing neurons in the rat arcuate nucleus. Neuroscience Letters, 321(3), 157-160. Retrieved from http://www.ncbi.nlm.nih.gov/pubmed/11880196

Ludwig, D., Peterson, K., \& Gortmaker, S. (2001). Relation between consumption of sugar-sweetened drinks and childhood obesity: a prospective, observational analysis. Lancet, 357(9255), 505-508. doi:10.1016/S0140-6736(00)04041-1

Lutter, M., Sakata, I., Osborne-Lawrence, S., Rovinsky, S., Anderson, J., Jung, S., ... Zigman, J. (2008). The orexigenic hormone ghrelin defends against depressive symptoms of chronic stress. Nature Neuroscience, 11(7), 752-753. doi:10.1038/nn.2139

Malik, S., McGlone, F., Bedrossian, D., \& Dagher, A. (2008). Ghrelin modulates brain activity in areas that control appetitive behavior. Cell Metabolism, 7(5), 400-409. doi:10.1016/j.cmet.2008.03.007

Mani, B., Walker, A., Lopez Soto, E., Raingo, J., Lee, C., Perello, M., ... Zigman, J. (2014). Neuroanatomical characterization of a growth hormone secretagogue receptor-green fluorescent protein reporter mouse. The Journal of Comparative Neurology. doi:10.1002/cne.

Maric, T., Sedki, F., Ronfard, B., Chafetz, D., \& Shalev, U. (2012). A limited role for ghrelin in heroin self-administration and food deprivation-induced reinstatement of heroin seeking in rats. Addiction Biology, 17(3), 613-622. doi:10.1111/j.13691600.2011.00396.x

Marinelli, M., \& Piazza, P. (2002). Interaction between glucocorticoid hormones, stress and psychostimulant drugs. The European Journal of Neuroscience, 16(3), 387-394. Retrieved from http://www.ncbi.nlm.nih.gov/pubmed/12193179

Martín-García, E., Burokas, A., Kostrzewa, E., Gieryk, A., Korostynski, M., Ziolkowska, B., ... Maldonado, R. (2011). New operant model of reinstatement of food-seeking behavior in mice. Psychopharmacology, 215(1), 49-70. doi:10.1007/s00213-0102110-6

McDonald, A. (1987). Organization of amygdaloid projections to the mediodorsal thalamus and prefrontal cortex: a fluorescence retrograde transport study in the rat. The Journal of Comparative Neurology, 262(1), 46-58. doi:10.1002/cne.902620105 
McFarland, K., \& Kalivas, P. (2001). The circuitry mediating cocaine-induced reinstatement of drug-seeking behavior. Journal of Neuroscience, 21(21), 86558663. Retrieved from http://www.ncbi.nlm.nih.gov/pubmed/11606653

Merkestein, M., Brans, M., Luijendijk, M., de Jong, J., Egecioglu, E., Dickson, S., \& Adan, R. (2012). Ghrelin mediates anticipation to a palatable meal in rats. Obesity, 20(5), 963-971. doi:10.1038/oby.2011.389

Mirenowicz, J., \& Schultz, W. (1996). Preferential activation of midbrsin dopamine neurons by appetitive rather than aversive stimuli. Nature, 379, 449-451.

Mistlberger, R. (1994). Circadian food-anticipatory activity: formal models and physiological mechanisms. Neuroscience and Biobehavioral Reviews, 18(2), 171195. Retrieved from http://www.ncbi.nlm.nih.gov/pubmed/8058212

Miura, T., Maruyama, K., Shimakura, S., Kaiya, H., Uchiyama, M., Kangawa, K., ... Matsuda, K. (2007). Regulation of food intake in the goldfish by interaction between ghrelin and orexin. Peptides, 28(6), 1207-1213. doi:10.1016/j.peptides.2007.03.023

Mondal, M., Date, Y., Yamaguchi, H., Toshinai, K., Tsuruta, T., Kangawa, K., \& Nakazato, M. (2005). Identification of ghrelin and its receptor in neurons of the rat arcuate nucleus. Regulatory Peptides, 126(1-2), 55-59. doi:10.1016/j.regpep.2004.08.038

Mueller, D., Perdikaris, D., \& Stewart, J. (2002). Persistence and drug-induced reinstatement of a morphine-induced conditioned place preference. Behavioural Brain Research, 136(2), 389-397. Retrieved from http://www.ncbi.nlm.nih.gov/pubmed/12429400

Mueller, D., \& Stewart, J. (2000). Cocaine-induced conditioned place preference: reinstatement by priming injections of cocaine after extinction. Behavioural Brain Research, 115(1), 39-47. Retrieved from http://www.ncbi.nlm.nih.gov/pubmed/10996406

Nair, S., Adams-Deutsch, T., Epstein, D., \& Shaham, Y. (2009). The neuropharmacology of relapse to food seeking: methodology, main findings, and comparison with relapse to drug seeking. Progress in Neurobiology, 89(1), 18-45. doi:10.1016/j.pneurobio.2009.05.003

Nair, S., Adams-Deutsch, T., Pickens, C., Smith, D., \& Shaham, Y. (2009). Effects of the $\mathrm{MCH} 1$ receptor antagonist SNAP 94847 on high-fat food-reinforced operant responding and reinstatement of food seeking in rats. Psychopharmacology, 205(1), 129-140. doi:10.1007/s00213-009-1523-6

Nair, S., Golden, S., \& Shaham, Y. (2008). Differential effects of the hypocretin 1 receptor antagonist SB 334867 on high-fat food self-administration and 
reinstatement of food seeking in rats. British Journal of Pharmacology, 154(2), 406416. doi: $10.1038 /$ bjp. 2008.3

Nakazato, M., Murakami, N., Date, Y., Kojima, M., Matsuo, H., Kangawa, K., \& Matsukura, S. (2001). A role for ghrelin in the central regulation of feeding. Nature, 409(6817), 194-198. doi:10.1038/35051587

Naleid, A., Grace, M., Cummings, D., \& Levine, A. (2005). Ghrelin induces feeding in the mesolimbic reward pathway between the ventral tegmental area and the nucleus accumbens. Peptides, 26(11), 2274-2279. doi:10.1016/j.peptides.2005.04.025

Narita, M., Nagumo, Y., Hashimoto, S., Khotib, J., Miyatake, M., Sakurai, T., ... Suzuki, T. (2006). Direct involvement of orexinergic systems in the activation of the mesolimbic dopamine pathway and related behaviors induced by morphine. Journal of Neuroscience, 26(2), 398-405. doi:10.1523/JNEUROSCI.2761-05.2006

Nestler, E., \& Carlezon, W. (2006). The mesolimbic dopamine reward circuit in depression. Biological Psychiatry, 59(12), 1151-1159.

doi:10.1016/j.biopsych.2005.09.018

Nisembaum, L., de Pedro, N., Delgado, M., \& Isorna, E. (2014). Crosstalking between the "gut-brain" hormone ghrelin and the circadian system in the goldfish. Effects on clock gene expression and food anticipatory activity. General and Comparative Endocrinology, in press. doi:10.1016/j.ygcen.2014.03.016

Olds, J., \& Milner, P. (1954). Positive Reinforcement Produced by Electrical Stimulation of Septal Area and Other Regions of Rat Brain. Journal of Comparative and Physiological Psychology, 47, 419-427.

Oliver, G., \& Wardle, J. (1999). Perceived effects of stress on food choice. Physiology \& Behavior, 66(3), 511-515. Retrieved from http://www.ncbi.nlm.nih.gov/pubmed/10357442

Olszewski, P., Grace, M., Billington, C., \& Levine, A. (2003). Hypothalamic paraventricular injections of ghrelin: effect on feeding and c-Fos immunoreactivity. Peptides, 24(6), 919-923. doi:10.1016/S0196-9781(03)00159-1

Overduin, J., Figlewicz, D., Bennett-jay, J., Kittleson, S., \& Cummings, D. (2012). Ghrelin increases the motivation to eat, but does not alter food palatability. American Journal of Physiology. Regulatory, Integrative and Comparative Physiology, 303(3), 259-269. doi:10.1152/ajpregu.00488.2011

Park, T., \& Carr, K. (1998). Neuroanatomical patterns of fos-like immunoreactivity induced by a palatable meal and meal-paired environment in saline- and naltrexonetreated rats. Brain Research, 805(1-2), 169-180. Retrieved from http://www.ncbi.nlm.nih.gov/pubmed/9733960 
Patterson, Z., Ducharme, R., Anisman, H., \& Abizaid, A. (2010). Altered metabolic and neurochemical responses to chronic unpredictable stressors in ghrelin receptordeficient mice. The European Journal of Neuroscience, 32(4), 632-639. doi:10.1111/j.1460-9568.2010.07310.x

Patton, D., \& Mistlberger, R. (2013). Circadian adaptations to meal timing: neuroendocrine mechanisms. Frontiers in Neuroscience, 7, 185. doi:10.3389/fnins.2013.00185

Paxinos, G., \& Watson, C. (1998). The Rat Brain in Stereotaxic Coordinates (4th editio.). Academic Press, Incorporated.

Peciña, S., Cagniard, B., Berridge, K., Aldridge, J., \& Zhuang, X. (2003). Hyperdopaminergic mutant mice have higher "wanting" but not "liking" for sweet rewards. The Journal of Neuroscience, 23(28), 9395-9402. Retrieved from http://www.ncbi.nlm.nih.gov/pubmed/14561867

Pecoraro, N., Reyes, F., Gomez, F., Bhargava, A., \& Dallman, M. (2004). Chronic stress promotes palatable feeding, which reduces signs of stress: feedforward and feedback effects of chronic stress. Endocrinology, 145(8), 3754-3762. doi:10.1210/en.20040305

Perello, M., Sakata, I., Birnbaum, S., Chuang, J., Osborne-Lawrence, S., Rovinsky, S., ... Zigman, J. (2010). Ghrelin increases the rewarding value of high-fat diet in an orexin-dependent manner. Biological Psychiatry, 67(9), 880-886.

doi:10.1016/j.biopsych.2009.10.030

Perelló, M., \& Zigman, J. (2012). The role of ghrelin in reward-based eating. Biological Psychiatry, 72(5), 347-353. doi:10.1016/j.biopsych.2012.02.016

Petrovich, G., \& Gallagher, M. (2003). Amygdala Subsystems and Control of Feeding Behavior by Learned Cues. Annals of the New York Academy of Sciences, 985, 251263.

Petrovich, G., Holland, P., \& Gallagher, M. (2005). Amygdalar and prefrontal pathways to the lateral hypothalamus are activated by a learned cue that stimulates eating. Journal of Neuroscience, 25(36), 8295-8302. doi:10.1523/JNEUROSCI.248005.2005

Pfluger, P., Castañeda, T., Heppner, K., Strassburg, S., Kruthaupt, T., Chaudhary, N., ... Perez-Tilve, D. (2011). Ghrelin, peptide YY and their hypothalamic targets differentially regulate spontaneous physical activity. Physiology \& Behavior, 105(1), 52-61. doi:10.1016/j.physbeh.2011.04.025

Pfluger, P., Kirchner, H., Gunnel, S., Schrott, B., Perez-Tilve, D., Fu, S., ... Tscho, M. (2008). Simultaneous deletion of ghrelin and its receptor increases motor activity 
and energy expenditure. American Journal of Physiology. Gastrointestinal and Liver Physiology, 294, 610-618. doi:10.1152/ajpgi.00321.2007.

Piazza, P., \& Moal, M. (1998). The role of stress in drug self-administration. Trends in Pharmacological Sciences, 19(2), 67-74.

Pijlman, F., Wolterink, G., \& Ree, J. (2003). Physical and emotional stress have differential effects on preference for saccharine and open field behaviour in rats. Behavioural Brain Research, 139, 131-138.

Pinto, S., Roseberry, A., Liu, H., Diano, S., Shanabrough, M., Cai, X., ... Horvath, T. (2004). Rapid rewiring of arcuate nucleus feeding circuits by leptin. Science, 304(5667), 110-115. doi:10.1126/science.1089459

Plaza-Zabala, A., Maldonado, R., \& Berrendero, F. (2012). The hypocretin/orexin system: implications for drug reward and relapse. Molecular Neurobiology, 45(3), 424-39. doi:10.1007/s12035-012-8255-Z

Richards, J., Simms, J., Steensland, P., Taha, S., Borgland, S., Bonci, A., \& Bartlett, S. (2008). Inhibition of orexin-1/hypocretin-1 receptors inhibits yohimbine-induced reinstatement of ethanol and sucrose seeking in Long-Evans rats. Psychopharmacology, 199(1), 109-117. doi:10.1007/s00213-008-1136-5

Richardson, N., \& Gratton, A. (1998). Changes in medial prefrontal cortical dopamine levels associated with response-contingent food reward: an electrochemical study in rat. The Journal of Neuroscience, 18(21), 9130-9138. Retrieved from http://www.ncbi.nlm.nih.gov/pubmed/9787015

Richardson, N., \& Roberts, D. (1996). Progressive ratio schedules in drug selfadministration studies in rats: a method to evaluate reinforcing efficacy. Journal of Neuroscience Methods, 66(1), 1-11. Retrieved from http://www.ncbi.nlm.nih.gov/pubmed/8794935

Robbins, T., \& Arnsten, A. (2010). The neuropsychopharmacology and fronto-executive function: monoaminergic modulation. Annual Review of Neuroscience, 32, 267-287. doi:10.1146/annurev.neuro.051508.135535.The

Roitman, M., Stuber, G., Phillips, P., Wightman, R., \& Carelli, R. (2004). Dopamine operates as a subsecond modulator of food seeking. The Journal of Neuroscience, 24(6), 1265-1271. doi:10.1523/JNEUROSCI.3823-03.2004

Roma, P., \& Riley, A. (2005). Apparatus bias and the use of light and texture in place conditioning. Pharmacology, Biochemistry, and Behavior, 82(1), 163-169. doi:10.1016/j.pbb.2005.08.004 
Romero, A., Kirchner, H., Heppner, K., Pfluger, P., Tschöp, M., \& Nogueiras, R. (2010). GOAT: the master switch for the ghrelin system? European Journal of Endocrinology, 163(1), 1-8. doi:10.1530/EJE-10-0099

Rorabaugh, J., Stratford, J., \& Zahniser, N. (2014). A Relationship between reduced nucleus accumbens shell and enhanced lateral hypothalamic orexin neuronal activation in long-term fructose bingeing behavior. PloS One, 9(4), e95019. doi:10.1371/journal.pone.0095019

Rouach, V., Bloch, M., Rosenberg, N., Gilad, S., Limor, R., Stern, N., \& Greenman, Y. (2007). The acute ghrelin response to a psychological stress challenge does not predict the post-stress urge to eat. Psychoneuroendocrinology, 32(6), 693-702. doi:10.1016/j.psyneuen.2007.04.010

Sakurai, T., Amemiya, A., Ishii, M., Matsuzaki, I., Chemelli, R., Tanaka, H., ... Yanagisawa, M. (1998). Orexins and orexin receptors: a family of hypothalamic neuropeptides and $\mathrm{G}$ protein-coupled receptors that regulate feeding behavior. Cell, 92(4), 573-785. Retrieved from http://www.ncbi.nlm.nih.gov/pubmed/9491897

Sakurai, T., \& Mieda, M. (2011). Connectomics of orexin-producing neurons: interface of systems of emotion, energy homeostasis and arousal. Trends in Pharmacological Sciences, 32(8), 451-462. doi:10.1016/j.tips.2011.03.007

Sanchis-segura, C., \& Spanagel, R. (2006). Behavioural assessment of drug reinforcement and addictive features in rodents : an overview. Addiction Biology, 11, 2-38. doi:10.1111/j.1355-6215.2006.00012.x

Sapolsky, R. (2004). Why zebras don't get ulcers (pp. 1-212). New York: W.H. Freeman and Company.

Schellekens, H., Finger, B., Dinan, T., \& Cryan, J. (2012). Ghrelin signalling and obesity: at the interface of stress, mood and food reward. Pharmacology \& Therapeutics, 135(3), 316-326. doi:10.1016/j.pharmthera.2012.06.004

Schneeberger, M., Gomis, R., \& Claret, M. (2014). Hypothalamic and brainstem neuronal circuits controlling homeostatic energy balance. The Journal of Endocrinology, 220(2), 25-46. doi:10.1530/JOE-13-0398

Schneider, E., Rada, P., Darby, R., Leibowitz, S., \& Hoebel, B. (2007). Orexigenic peptides and alcohol intake: differential effects of orexin, galanin, and ghrelin. Alcoholism, Clinical and Experimental Research, 31(11), 1858-1865. doi:10.1111/j.1530-0277.2007.00510.x

Schultz, W., Apicella, P., \& Ljungberg, T. (1993). Responses of monkey dopamine neurons to reward and conditioned stimuli during successive steps of learning a 
delayed response task. Journal of Neuroscience, 13(3), 900-913. Retrieved from http://www.ncbi.nlm.nih.gov/pubmed/8441015

Schüssler, P., Kluge, M., Yassouridis, A., Dresler, M., Uhr, M., \& Steiger, A. (2012). Ghrelin levels increase after pictures showing food. Obesity, 20(6), 1212-1217. doi:10.1038/oby.2011.385

Schwartz, M., Woods, S., Porte, D., Seeley, R., \& Baskin, D. (2000). Central nervous system control of food intake. Nature, 404(6778), 661-671.

Scott, V., McDade, D., \& Luckman, S. (2007). Rapid changes in the sensitivity of arcuate nucleus neurons to central ghrelin in relation to feeding status. Physiology \& Behavior, 90(1), 180-185. doi:10.1016/j.physbeh.2006.09.026

See, R., \& Waters, R. (2011). Pharmacologically-induced stress: a cross-species probe for translational research in drug addiction and relapse. American Journal of Translational Research, 3(1), 81-89. Retrieved from http://www.pubmedcentral.nih.gov/articlerender.fcgi?artid=2981428\&tool=pmcentr ez\&rendertype $=$ abstract

Self, D., \& Nestler, E. (1998). Relapse to drug-seeking: neural and molecular mechanisms. Drug and Alcohol Dependence, 51(1-2), 49-60. Retrieved from http://www.ncbi.nlm.nih.gov/pubmed/9716929

Shaham, Y., Shalev, U., Lu, L., De Wit, H., \& Stewart, J. (2003). The reinstatement model of drug relapse: history, methodology and major findings. Psychopharmacology, 168(1-2), 3-20. doi:10.1007/s00213-002-1224-x

Shalev, U., Grimm, J., \& Shaham, Y. (2002). Neurobiology of relapse to heroin and cocaine seeking: a review. Pharmacological Reviews, 54(1), 1-42. Retrieved from http://www.ncbi.nlm.nih.gov/pubmed/11870259

Shalev, U., Marinelli, M., Baumann, M., Piazza, P., \& Shaham, Y. (2003). The role of corticosterone in food deprivation-induced reinstatement of cocaine seeking in the rat. Psychopharmacology, 168(1-2), 170-176. doi:10.1007/s00213-002-1200-5

Shalev, U., Yap, J., \& Shaham, Y. (2001). Leptin attenuates acute food deprivationinduced relapse to heroin seeking. Journal of Neuroscience, 21(4), 129. Retrieved from http://www.ncbi.nlm.nih.gov/pubmed/11160414

Shepard, J., Barron, K., \& Myers, D. (2000). Corticosterone delivery to the amygdala increases corticotropin-releasing factor mRNA in the central amygdaloid nucleus and anxiety-like behavior. Brain Research, 861(2), 288-295. Retrieved from http://www.ncbi.nlm.nih.gov/pubmed/10760490 
Shimbara, T., Mondal, M., Kawagoe, T., Toshinai, K., Koda, S., Yamaguchi, H., ... Nakazato, M. (2004). Central administration of ghrelin preferentially enhances fat ingestion. Neuroscience Letters, 369(1), 75-79. doi:10.1016/j.neulet.2004.07.060

Shioda, S., Takenoya, F., Yagi, M., Wang, L., Hori, Y., \& Kageyama, H. (2008). Neural networks of several novel neuropeptides involved in feeding regulation. Nutrition, 24(9), 848-53. doi:10.1016/j.nut.2008.06.016

Skibicka, K., Hansson, C., Alvarez-Crespo, M., Friberg, P., \& Dickson, S. (2011). Ghrelin directly targets the ventral tegmental area to increase food motivation. Neuroscience, 180, 129-137. doi:10.1016/j.neuroscience.2011.02.016

Skibicka, K., Hansson, C., Egecioglu, E., \& Dickson, S. (2012). Role of ghrelin in food reward : impact of ghrelin on sucrose self-administration and mesolimbic dopamine and acetylcholine receptor gene expression. Addiction Biology, 17(1), 95-107. doi:10.1111/j.1369-1600.2010.00294.x

Skibicka, K., Shirazi, R., Hansson, C., \& Dickson, S. (2012). Ghrelin interacts with neuropeptide Y Y1 and opioid receptors to increase food reward. Endocrinology, 153(3), 1194-1205. doi:10.1210/en.2011-1606

Skibicka, K., Shirazi, R., Rabasa-papio, C., Alvarez-crespo, M., Neuber, C., Vogel, H., \& Dickson, S. (2013). Divergent circuitry underlying food reward and intake effects of ghrelin : Dopaminergic VTA-accumbens projection mediates ghrelin's effect on food reward but not food intake. Neuropharmacology, 73, 274-283.

doi:10.1016/j.neuropharm.2013.06.004

Smith, G., Jerome, C., \& Norgren, R. (1985). Afferent axons in abdominal vagus mediate satiety effect of cholecystokinin in rats. The American Journal of Physiology, 249(2), 638-641. Retrieved from http://www.ncbi.nlm.nih.gov/pubmed/4061684

Smith, K., Tindell, A., Aldridge, J., \& Berridge, K. (2009). Ventral pallidum roles in reward and motivation. Behavioural Brain Research, 196(2), 155-167. doi:10.1016/j.bbr.2008.09.038

Smith, R., See, R., \& Aston-Jones, G. (2009). Orexin/hypocretin signaling at the orexin 1 receptor regulates cue-elicited cocaine-seeking. The European Journal of Neuroscience, 30(3), 493-503. doi:10.1111/j.1460-9568.2009.06844.x

Spinedi, E., Voirol, M., Verdumo, C., Giacominni, M., Pralong, F., \& Gaillard, R. (2006). Hypothalamic ghrelin treatment modulates NPY- but not CRH-ergic activity in adrenalectomized rats subjected to food restriciton. Endocrine, 29(3), 477-484.

Stambor, Z. (2006). Stressed out nation. Monitor on Psychology, 37(4), 28. doi:10.1037/e494972006-019 
Steffensen, S., Lee, R., Stobbs, S., \& Henriksen, S. (2001). Responses of ventral tegmental area GABA neurons to brain stimulation reward. Brain Research, 906(12), 190-197. Retrieved from http://www.ncbi.nlm.nih.gov/pubmed/11430879

Steketee, J., \& Kalivas, P. (2011). Drug Wanting : Behavioral Sensitization and Relapse to Drug-Seeking Behavior. Pharmacological Reviews, 63(2), 348-365. doi:10.1124/pr.109.001933.remains

Stephan, F. (2002). The "other" circadian system: food as a Zeitgeber. Journal of Biological Rhythms, 17(4), 284-292. Retrieved from http://www.ncbi.nlm.nih.gov/pubmed/12164245

Stewart, J. (1984). Reinstatement of heroin and cocaine self-administration behavior in the rat by intracerebral application of morphine in the ventral tegmental area. Pharmacology, Biochemistry, and Behavior, 20(6), 917-923. Retrieved from http://www.ncbi.nlm.nih.gov/pubmed/6463075

Strekalova, T., Spanagel, R., Dolgov, O., \& Bartsch, D. (2005). Stress-induced hyperlocomotion as a confounding factor in anxiety and depression models in mice. Behavioural Pharmacology, 16(3), 171-180. Retrieved from http://www.ncbi.nlm.nih.gov/pubmed/15864072

Stutzmann, G., McEwen, B., \& LeDoux, J. (1998). Serotonin modulation of sensory inputs to the lateral amygdala: dependency on corticosterone. Journal of Neuroscience, 18(22), 9529-9538. Retrieved from http://www.ncbi.nlm.nih.gov/pubmed/9801389

Sugino, T., Hasegawa, Y., Kikkawa, Y., Yamaura, J., Yamagishi, M., Kurose, Y., ... Terashima, Y. (2002). A transient ghrelin surge occurs just before feeding in a scheduled meal-fed sheep. Biochemical and Biophysical Research Communications, 295(2), 255-260. Retrieved from http://www.ncbi.nlm.nih.gov/pubmed/12150940

Sun, Y., Ahmed, D., Smith, R., \& Zivkovic, B. (2003). Deletion of Ghrelin Impairs neither Growth nor Appetite. Neuroscience Letters, 23(22), 7973-7981. doi:10.1128/MCB.23.22.7973

Sun, Y., Butte, N., Garcia, J., \& Smith, R. (2008). Characterization of adult ghrelin and ghrelin receptor knockout mice under positive and negative energy balance. Endocrinology, 149(2), 843-850. doi:10.1210/en.2007-0271

Sun, Y., Wang, P., Zheng, H., \& Smith, R. (2004). Ghrelin stimulation of growth hormone release and appetite is mediated through the growth hormone secretagogue receptor. PNAS, 101(13), 4679-4684. doi:10.1073/pnas.0305930101

Tanaka, C., Asakawa, A., Ushikai, M., Sakoguchi, T., Amitani, H., Terashi, M., ... Inui, A. (2009). Comparison of the anorexigenic activity of CRF family peptides. 
Biochemical and Biophysical Research Communications, 390(3), 887-891. doi:10.1016/j.bbrc.2009.10.069

Tang-Christensen, M., Vrang, N., Ortmann, S., Bidlingmaier, M., Horvath, T., \& Tschöp, M. (2004). Central administration of ghrelin and agouti-related protein (83-132) increases food intake and decreases spontaneous locomotor activity in rats. Endocrinology, 145(10), 4645-4652. doi:10.1210/en.2004-0529

Tassone, F., Broglio, F., Destefanis, S., Rovere, S., Benso, A., Gottero, C., ... Maccario, M. (2003). Neuroendocrine and metabolic effects of acute ghrelin administration in human obesity. The Journal of Clinical Endocrinology and Metabolism, 88(11), 5478-5483. doi:10.1210/jc.2003-030564

Tataranni, P., Larson, D., Snitker, S., Young, J., Flatt, J., \& Ravussin, E. (1996). Effects of glucocorticoids on energy metabolism and food intake in humans. The American Journal of Physiology: Endocrinology and Metabolism, 271, 317-325. Retrieved from http://www.ncbi.nlm.nih.gov/pubmed/8770026

Tessari, M., Catalano, A., Pellitteri, M., Di Francesco, C., Marini, F., Gerrard, P., ... Melotto, S. (2007). Correlation between serum ghrelin levels and cocaine-seeking behaviour triggered by cocaine-associated conditioned stimuli in rats. Addiction Biology, 12(1), 22-29. doi:10.1111/j.1369-1600.2007.00052.x

Toshinai, K., Date, Y., Murakami, N., Shimada, M., Mondal, M., Shimbara, T., ... Nakazato, M. (2003). Ghrelin-induced food intake is mediated via the orexin pathway. Endocrinology, 144(4), 1506-1512. doi:10.1210/en.2002-220788

Tschöp, M., Smiley, D., \& Heiman, M. (2000). Ghrelin induces adiposity in rodents. Nature, 407(6806), 908-913. doi:10.1038/35038090

Tschöp, M., Wawarta, R., Riepl, R., Friedrich, S., Bidlingmaier, M., Landgraf, R., \& Folwaczny, C. (2001). Post-prandial decrease of circulating human ghrelin levels. Journal of Endocrinological Investigation, 24(6), 19-21. Retrieved from http://www.ncbi.nlm.nih.gov/pubmed/11434675

Tsigos, C., \& Chrousos, G. (2002). Hypothalamic-pituitary-adrenal axis, neuroendocrine factors and stress. Journal of Psychosomatic Research, 53(4), 865-871. Retrieved from http://www.ncbi.nlm.nih.gov/pubmed/12377295

Tsujino, N., \& Sakurai, T. (2009). Orexin / hypocretin : A neuropeptide at the interface of sleep, energy homeostasis , and reward system. Pharmacological Reviews, 61(2), 162-176. doi:10.1124/pr.109.001321.162

Tzschentke, T. (2007). Measuring reward with the conditioned place preference (CPP) paradigm: update of the last decade. Addiction Biology, 12(3-4), 227-462. doi:10.1111/j.1369-1600.2007.00070.x 
Valdivia, S., Patrone, A., Reynaldo, M., \& Perello, M. (2014). Acute high fat diet consumption activates the mesolimbic circuit and requires orexin signaling in a mouse model. PloS One, 9(1), e87478. doi:10.1371/journal.pone.0087478

Verbaeys, I., Tolle, V., Swennen, Q., Zizzari, P., Buyse, J., Epelbaum, J., \& Cokelaere, M. (2011). Scheduled feeding results in adipogenesis and increased acylated ghrelin. American Journal of Physiology. Endocrinology and Metabolism, 300, 1103-1111. doi:10.1152/ajpendo.00551.2010.

Verhagen, L., Egecioglu, E., Luijendijk, M., Hillebrand, J., Adan, R., \& Dickson, S. (2011). Acute and chronic suppression of the central ghrelin signaling system reveals a role in food anticipatory activity. European Neuropsychopharmacology, 21(5), 384-392. doi:10.1016/j.euroneuro.2010.06.005

Waddington Lamont, E., Bruton, J., Blum, I., \& Abizaid, A. (2014). Ghrelin receptorknockout mice display alterations in circadian rhythms of activity and feeding under constant lighting conditions. The European Journal of Neuroscience, 39(2), 207217. doi: $10.1111 /$ ejn. 12390

Walker, A., Ibia, I., \& Zigman, J. (2012). Disruption of cue-potentiated feeding in mice with blocked ghrelin signaling. Physiology \& Behavior, 108, 34-43. doi:10.1016/j.physbeh.2012.10.003

Wang, B., You, Z., Rice, K., \& Wise, R. (2007). Stress-induced relapse to cocaine seeking: roles for the $\mathrm{CRF}(2)$ receptor and CRF-binding protein in the ventral tegmental area of the rat. Psychopharmacology, 193(2), 283-294. doi:10.1007/s00213-007-0782-3

Wang, B., You, Z., \& Wise, R. (2009). Reinstatement of cocaine seeking by hypocretin (orexin) in the ventral tegmental area: independence from the local corticotropinreleasing factor network. Biological Psychiatry, 65(10), 857-862. doi:10.1016/j.biopsych.2009.01.018

Wang, L., Saint-Pierre, D., \& Taché, Y. (2002). Peripheral ghrelin selectively increases Fos expression in neuropeptide $\mathrm{Y}$ - synthesizing neurons in mouse hypothalamic arcuate nucleus. Neuroscience Letters, 325(1), 47-51. Retrieved from http://www.ncbi.nlm.nih.gov/pubmed/12023064

Weinberg, Z., Nicholson, M., \& Currie, P. (2011). 6-Hydroxydopamine lesions of the ventral tegmental area suppress ghrelin's ability to elicit food-reinforced behavior. Neuroscience Letters, 499(2), 70-73. doi:10.1016/j.neulet.2011.05.034

Weiss, F. (2005). Neurobiology of craving, conditioned reward and relapse. American Journal of Psychiatry, 5(1), 9-19. doi:10.1016/j.coph.2004.11.001 
Wellman, P., Clifford, P., Rodriguez, J., Hughes, S., Di Francesco, C., Melotto, S., ... Gozzi, A. (2012). Brain reinforcement system function is ghrelin dependent: studies in the rat using pharmacological fMRI and intracranial self-stimulation. Addiction Biology, 17(5), 908-919. doi:10.1111/j.1369-1600.2011.00392.x

Wellman, P., Davis, K., \& Nation, J. (2005). Augmentation of cocaine hyperactivity in rats by systemic ghrelin. Regulatory Peptides, 125(1-3), 151-154.

doi:10.1016/j.regpep.2004.08.013

Wellman, P., Hollas, C., \& Elliott, A. (2008). Systemic ghrelin sensitizes cocaineinduced hyperlocomotion in rats. Regulatory Peptides, 146, 33-37. doi:10.1016/j.regpep.2007.07.007

Westerink, B., \& Kwint, H. (1996). The Pharmacology of Mesolimbic Dopamine Neurons : A Dual-Probe Microdialysis Study in the Ventral Tegmental and Nucleus Accumbens of the Rat Brain. Journal of Neuroscience, 76(8), 2605-2611.

Wing, R., \& Phelan, S. (2005). Long-term weight loss maintenance. The American Journal of Clinical Nutrition, 82, 222S-225S. Retrieved from http://www.ncbi.nlm.nih.gov/pubmed/16002825

Wise, R. (2002). Brain reward circuitry : Insights from unsensed incentives. Neuron, 36, 229-240.

Wise, R. (2004). Dopamine, learning and motivation. Nature Reviews: Neuroscience, 5(6), 483-494. doi:10.1038/nrn1406

Wise, R. (2006). Role of brain dopamine in food reward and reinforcement. Philosophical Transactions of the Royal Society of London. Series B, Biological Sciences, 361(1471), 1149-1158. doi:10.1098/rstb.2006.1854

Woods, S. (1991). The eating paradox: how we tolerate food. Psychological Review, 98(4), 488-505. Retrieved from http://www.ncbi.nlm.nih.gov/pubmed/1961770

Wortley, K., Anderson, K., Garcia, K., Murray, J., Malinova, L., Liu, R., ... Sleeman, M. (2004). Genetic deletion of ghrelin does not decrease food intake but influences metabolic fuel preference. PNAS, 101(21), 8227-8232. doi:10.1073/pnas.0402763101

Wortley, K., Rincon, J., Murray, J., Garcia, K., Iida, K., Thorner, M., \& Sleeman, M. (2005). Absence of ghrelin protects against early-onset obesity. The Journal of Clinical Investigation, 115(12), 3573-3578. doi:10.1172/JCI26003.feeding

Wren, A., Small, C., Abbott, C., Dhillo, W., Seal, L., Cohen, M., ... Bloom, S. (2001). Ghrelin causes hyperphagia and obesity in rats. Diabetes, 50(11), 2540-2547. Retrieved from http://www.ncbi.nlm.nih.gov/pubmed/11679432 
Yamanaka, A., Kunii, K., Nambu, T., Tsujino, N., Sakai, A., Matsuzaki, I., ... Sakurai, T. (2000). Orexin-induced food intake involves neuropeptide Y pathway. Brain Research, 859(2), 404-409. Retrieved from http://www.ncbi.nlm.nih.gov/pubmed/10719096

Young, E., \& Williams, C. (2010). Valence dependent asymmetric release of norepinephrine in the basolateral amygdala. Behavioral Neuroscience, 124(5), 633644. doi: $10.1037 / \mathrm{a} 0020885$

Yun, I., Wakabayashi, K., Fields, H., \& Nicola, S. (2004). The ventral tegmental area is required for the behavioral and nucleus accumbens neuronal firing responses to incentive cues. Journal of Neuroscience, 24(12), 2923-2933. doi:10.1523/JNEUROSCI.5282-03.2004

Zan, Y., Haag, J., Chen, K., Shepel, L., Wigington, D., Wang, Y., ... Gould, M. (2003). Production of knockout rats using ENU mutagenesis and a yeast-based screening assay. Nature Biotechnology, 21(6), 645-651. doi:10.1038/nbt830

Zellner, D., Loaiza, S., Gonzalez, Z., Pita, J., Morales, J., Pecora, D., \& Wolf, A. (2006). Food selection changes under stress. Physiology \& Behavior, 87(4), 789-793. doi:10.1016/j.physbeh.2006.01.014

Zhao, T., Liang, G., Li, R., Xie, X., Sleeman, M., Murphy, A., ... Brown, M. (2010). Ghrelin O-acyltransferase (GOAT) is essential for growth hormone-mediated survival of calorie-restricted mice. PNAS, 107(16), 7467-7472.

doi:10.1073/pnas.1002271107

Zigman, J., Jones, J., Lee, C., Saper, C., \& Elmquist, J. (2006). Expression of ghrelin receptor mRNA in the rat and the mouse brain. The Journal of Comparative Neurology, 494(3), 528-548. doi:10.1002/cne.20823

Zigman, J., Nakano, Y., Coppari, R., Balthasar, N., Marcus, J., Lee, C., ... Elmquist, J. (2005). Mice lacking ghrelin receptors resist the development of diet-induced obesity. The Journal of Clinical Investigation, 115(12), 3564-3572.

doi:10.1172/JCI26002.3564 